\title{
Angle, spin and depth resolved photoelectron spectroscopy on quantum materials
}

\author{
Phil D.C. King, ${ }^{\dagger}$ Silvia Picozzi, ${ }^{\ddagger}$ Russell G. Egdell, ${ }^{\top}$ and Giancarlo Panaccione ${ }^{*, \S}$ \\ $\dagger S U P A$, School of Physics and Astronomy,University of St. Andrews, St. Andrews KY16 \\ 9SS, United Kingdom \\ $\ddagger$ Consiglio Nazionale delle Ricerche, CNR-SPIN, Via dei Vestini 31, Chieti 66100, Italy \\ IDepartment of Chemistry, Inorganic Chemistry Laboratory, University of Oxford, South \\ Parks Road, Oxford OX1 3QR, U.K. \\ §Istituto Officina dei Materiali (IOM)-CNR, Laboratorio TASC, in Area Science Park, \\ S.S.14, Km 163.5, I-34149 Trieste, Italy \\ E-mail: panaccione@iom.cnr.it
}

\begin{abstract}
The role of X-ray based electron spectroscopies in determining chemical, electronic and magnetic properties of solids has been well known for several decades. A powerful approach is angle-resolved photoelectron spectroscopy, whereby the kinetic energy and angle of photoelectrons emitted from a sample surface are measured. This provides a direct measurement of the electronic band structure of crystalline solids. Moreover, it yields powerful insights into the electronic interactions at play within a material, and into the control of spin, charge and orbital degrees of freedom; central pillars of future solid state science. With strong recent focus on research of lower-dimensional materials and modified electronic behaviour at surfaces and interfaces, angle-resolved photoelectron spectroscopy has become a core technique in the study of quantum ma-
\end{abstract}


terials. In this review, we provide an introduction to the technique. Through examples from several topical materials systems, including topological insulators, transition metal dichalcogenides, and transition metal oxides, we highlight the types of information which can be obtained. We show how the combination of angle, spin, time and depth-resolved experiments are able to reveal 'hidden' spectral features, connected to semiconducting, metallic and magnetic properties of solids, as well as underlining the importance of dimensional effects in quantum materials.

\section{Contents}

1 Introduction $\quad 4$

1.1 Quantum Materials and Photoemission ............... 4

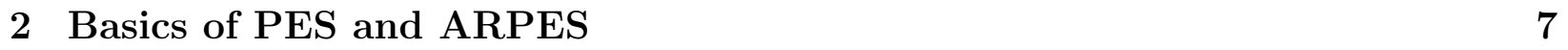

2.1 Historical background . . . . . . . . . . . . . . . . . . . . . . . . . 7

2.2 Principles of Photoelectron Spectroscopy . . . . . . . . . . . . . . . 8

2.3 Description and modelling of the ARPES process . . . . . . . . . . . . 9

2.4 Spectrometers: past and present . . . . . . . . . . . . . . 13

2.5 Use of light polarization in ARPES: symmetry of electronic states . . . . . . 16

2.6 Spin-resolved ARPES . . . . . . . . . . . . . . . . . 18

3 Comparison between ARPES and density functional theory 19

4 Depth-dependent information in photoemission $\quad 22$

4.1 Bulk sensitivity and HAXPES . . . . . . . . . . . . . . . 22

4.2 Use of cross sections in HAXPES . . . . . . . . . . . . . . . . . 25

4.3 Angular resolved HAXPES . . . . . . . . . . . . . . . . . . 27

5 Example materials systems $\quad 30$

5.1 Spin-orbit materials: non-trivial topology and Rashba effects . . . . . . . . . 30 
5.1 .1 Topological insulators . . . . . . . . . . . . . . . . . . . . 31

5.1 .2 Rashba materials . . . . . . . . . . . . . . . . . . . . 35

5.2 Transition-metal dichalcogenides . . . . . . . . . . . . . . . . . 41

5.2 .1 Chemical trends . . . . . . . . . . . . . . . . . . . . . . . 43

5.2 .2 Dimensional-crossovers . . . . . . . . . . . . . . . . . . . . . . . . . . . 49

5.2 .3 Spin-momentum locking . . . . . . . . . . . . . . . 56

5.3 Transition metal Oxides . . . . . . . . . . . . . . . . . 61

$5.3 .1 \quad$ In-gap and extended states at $E_{f} \ldots \ldots \ldots \ldots$

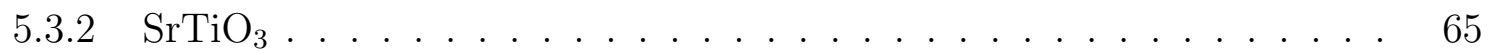

5.3 .3 Anatase $\mathrm{TiO}_{2} \ldots \ldots \ldots \ldots \ldots$

5.3 .4 Metallic oxides . . . . . . . . . . . . . . . . . . 71

$\begin{array}{llr}6 & \text { Future directions and technical challenges } & 76\end{array}$

6.1 Micro and Nano-ARPES . . . . . . . . . . . . . . . . . . . 77

6.2 Time Resolved ARPES . . . . . . . . . . . . . . . . . . . . . . . 79

6.3 Integrating growth and ARPES . . . . . . . . . . . 81

6.4 The future of spin-resolved ARPES . . . . . . . . . . . . . . . 81

7 Conclusions and Perspectives $\quad 82$

$\begin{array}{ll}\text { Biographies } & 83\end{array}$

$\begin{array}{lr}\text { Acknowledgement } & 84\end{array}$

$\begin{array}{lr}\text { References } & 85\end{array}$ 


\section{Introduction}

\subsection{Quantum Materials and Photoemission}

The collective behaviour of electrons in condensed matter is one of the central topics of modern science and technology. Collective quantum phenomena in materials arise from the interplay between quantum mechanics and interactions in many-particle systems - i.e. cooperative behaviours that cannot be predicted from the properties of individual electrons. In recent years, the study of emergent properties revealed a wealth of new systems, no longer restricted to those with strong electronic correlation, and a new definition has become necessary; 'quantum materials' provides such a definition.

The search for new states of matter is a central challenge at the intersection of basic and applied science, and a crucial crossroad for technological innovation. The last decades have seen staggering advances in the synthesis, growth and characterization of new quantum materials. The rapid progress in graphene synthesis and the methodologies developed in the growth of ultrathin layers, for example, has led to widespread exploration of an everincreasing array of two-dimensional (2D) quantum systems. Single layers of transition metal dichalcogenides (TMDs) can be stacked together to create an almost arbitrarily-complex array of new "meta-materials" with highly-tuneable material properties. ${ }^{1-4}$ Quintuple-layer semiconductors, long-since known as excellent thermoelectrics, turn out to host a non-trivial topological order in their bulk - so called topological insulators - giving rise to new types of surface states. ${ }^{5}$ Spin-orbit coupling plays an important role in such systems, but has emerged more broadly as a source of exotic quantum phenomena in materials containing high-Z atoms, from Rashba effects in chalcogenides and halides ${ }^{6}$ to spin-orbit assisted Mott insulating states in iridates. ${ }^{7,8}$ Meanwhile, creating heterostructures from transition-metal oxides - something long-since exploited in the fabrication of semiconductor devices - opens

powerful new opportunities to tune and tailor strongly-correlated phases of matter. ${ }^{9,10}$ More generally, almost any physical property changes at the surface or interface of a solid. The 
broken/lowered symmetry with respect to the $3 \mathrm{D}$ environment plays a crucial role not only in modifying many of the fundamental interactions, but also in generating new effects not present in their 3D-volume counterpart, providing substantial opportunity for controlling quantum electronic states.

The ability to directly measure the electronic structure is an essential prerequisite to understanding, creating, and ultimately designing new quantum materials, be it in bulk form, as few-layer heterostructures, or with modified surface properties. Only few analytical techniques can provide this information, and photoelectron spectroscopy (PES) is certainly among the most widespread utilised worldwide. It has been widely applied in the study of topological solids, magnetic and spintronic materials, correlated solids, 2D materials and more (Figure 1(a)). In this review, we will briefly describe photoelectron (or photoemission) spectroscopy and its relevant aspects, focusing in particular on specific selected results on quantum materials obtained via valence band and angle-resolved photoemission spectroscopy (ARPES), including spin-resolved ARPES. As a technique, it can span a remarkably wide range of energy scales (Figure 1(c)) - from the keV scale, to provide information on chemical composition, core-level binding energies, and charge states, through the eV scale to provide insight on valence orbital states and band dispersions, through the meV scale to yield insight on many-particle interactions, energy gaps, and the detailed fermiology of materials. The importance of ARPES in the analysis of quantum materials is often focussed on the later set, at energy scales comparable to the relevant interactions which are at play. In many cases competing and co-existing interactions, all on an energy scale of a few to tens or hundreds of meV occur, which is now accessible to ARPES measurements performed with high energy and angular resolution. Moreover, when dealing with dimensionality effects, energy-dependent measurements, as well as comparison with model calculations, may disentangle the 1D, 2D, 3D (or hybrid) character of electronic states.

The literature devoted to PES and ARPES is vast, and many outstanding books and review papers are already available on this subject, where the reader may find detailed 


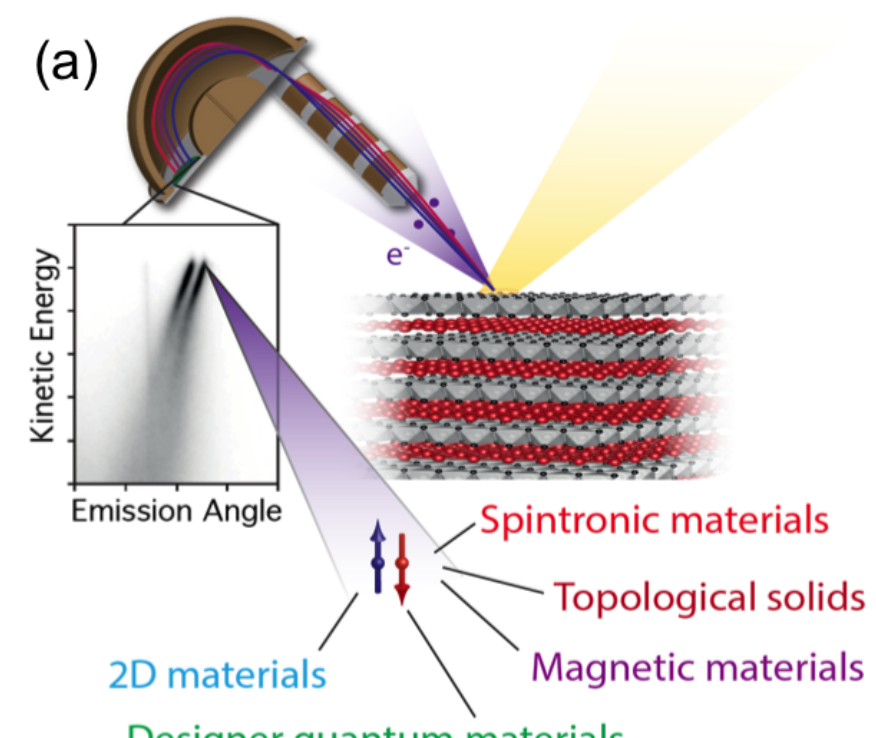

(b)

Photoemission spectrum Ekin

Electronic states

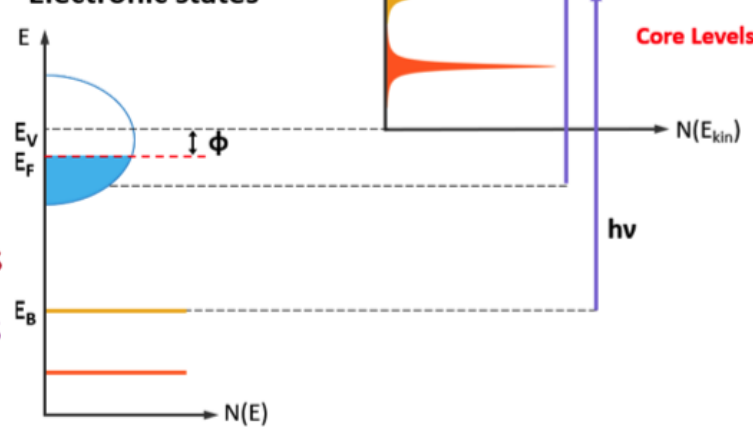

Kinetic energy (eV)

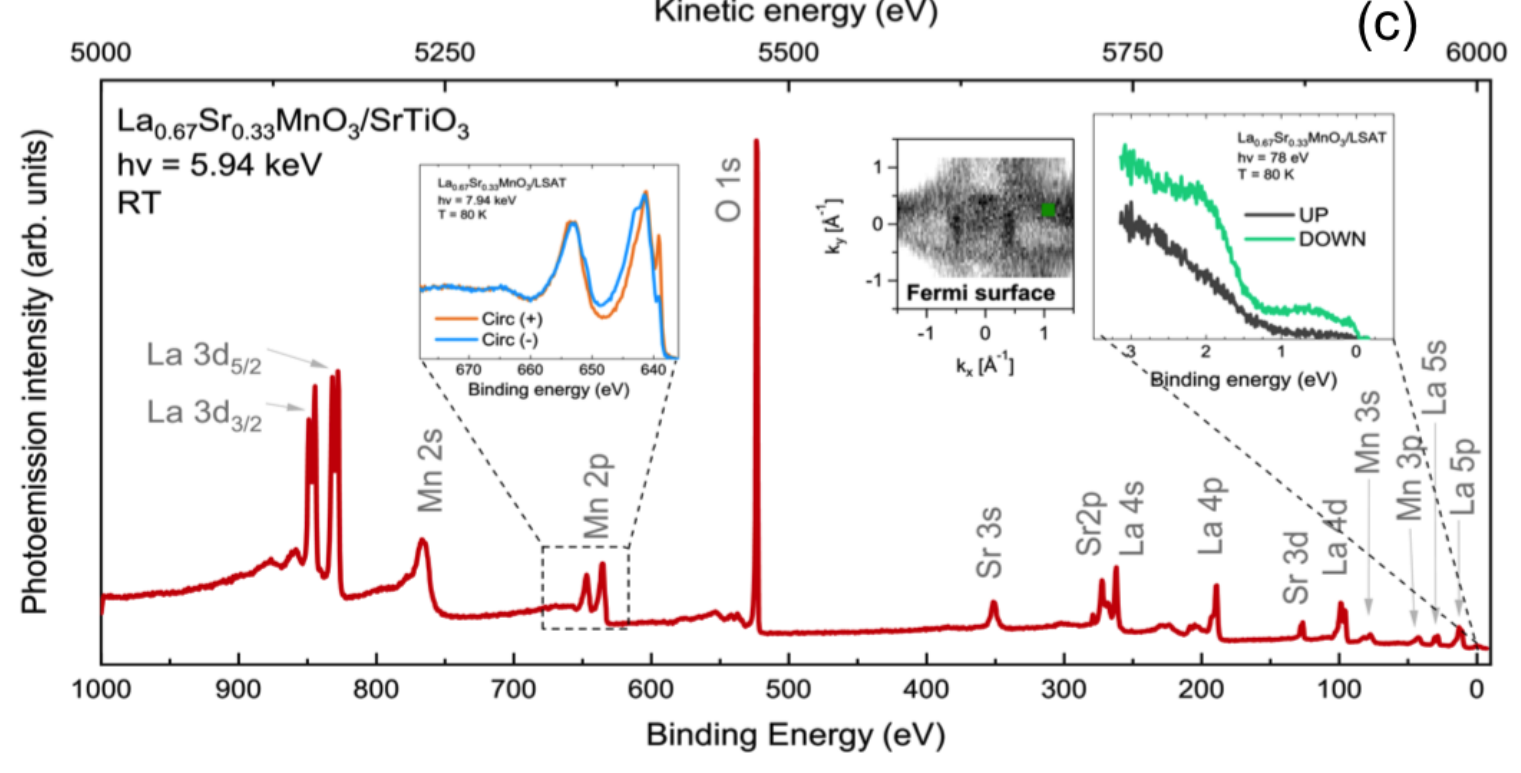

Figure 1: Panel (a): Principle of a photoemission experiment. Panel (b) Energetics and scheme of the energy level in the photoemission process. Panel (c) survey spectrum of a $\mathrm{La}_{0.67} \mathrm{Sr}_{0.33} \mathrm{MnO}_{3}$ sample. Top axis in kinetic energy and bottom axis in binding energy, following equation 1 . Peaks and symmetry of the core line are indicated. In the insets, from left to right: $2 p$ core level of Mn, measured with circular polarized light (left and right circular), showing the magnetic dichroic difference; Map of the fermi surface of LSMO; spin resolved photoemission spectra of the valence band with minority (black curve) and majority (green curve) intensities, defining spin unbalance in a ferromagnetic system 
insights, theory, and use examples of the technique. ${ }^{11-19}$ Here, we intend to focus on the use of ARPES to study quantum material properties of interest at the intersection of materials physics and materials chemistry. Our review has the following structure: we begin by giving a brief historical introduction and a summary of the relevant parameters of a photoemission experiment. We introduce angular-resolved photoemission, both from the experimental and theoretical point of view, as the door to access $k$-resolved information of solids. We will then present selected examples devoted to quantum materials. Finally, we will provide some insights into current trends and the open challenges and perspectives of the field.

\section{Basics of PES and ARPES}

\subsection{Historical background}

Photoelectron spectroscopy (PES) is based on the photoelectric effect, building on the Nobel prize winning work of Albert Einstein (1921, for 'his services to Theoretical physics, and especially his discovery of the law of the photoelectric effect'); Robert Andrew Millikan (1923, for 'his (experimental) work on the elementary charge of electricity and the photoelectric effect'; and Kai Manne Borje Siegbhan (1981, for 'his contribution to the development of high-resolution electron spectroscopy'). In the Nobel archive for 1981 one reads that 'electron spectroscopy had become a practical means of determining what the energies of the electrons were when originally trapped in the material'. ${ }^{20}$ The demonstration that the electronic binding energy of a core level depends upon the chemical environment of the atom in a solid is generally credited to Siegbahn, although a 'chemical shift' was first measured by Harold Roper Robinson in $1930 .{ }^{21}$ Siegbahn's work led to the acronym ESCA (Electron Spectroscopy for Chemical Analysis). ${ }^{22,23}$ His experiments involved X-ray excitation, hence the alternative acronym XPS (X-ray photoelectron spectroscopy). In parallel with the work on XPS, W.E. Spicer and others developed the photoemission technique using lower energy excitation in the vacuum ultraviolet, particularly using rare gas discharge lamps. Later the 
energy range between the two techniques was bridged using the tunable radiation available from synchrotrons. Initially this work was parasitic on the high energy physics experiments that provided the driver for construction of synchrotrons, but second generation and later synchrotrons were dedicated to production of synchrotron radiation. Nowadays, PES is used in thousands of laboratories worldwide, both in-house and at large scale synchrotron facilities, and is considered one of the most important and direct techniques to address the structural, electronic and magnetic properties of solids.

\subsection{Principles of Photoelectron Spectroscopy}

The direct link between the kinetic energy $\left(E_{\text {kin }}\right)$ of the photoelectron emitted from a solid and the energy of the photon absorbed $h v$, was first proposed by A. Einstein in the following equation: ${ }^{24}$

$$
h \nu=E_{B}+E_{k i n}+\Phi
$$

where $h \nu$ is the energy of the absorbed photon (with $h$ the Planck's constant), $E_{k i n}$ is the kinetic energy of the emitted photoelectron measured in the detector (i.e. an electron energy analyzer), $E_{B}$ is the binding energy relative to the Fermi level (or, more generally related to the last occupied electronic state), and $\Phi$ is the work function of the material, corresponding to the energy barrier the electrons must overcame to escape out of the solid (typically $<5$ $\mathrm{eV}) .{ }^{25}$ This simple formula explains why PES is of widespread use in solid-state science: the energetics (Figure 1(b)) gives i) a direct relationship between the measured kinetic energy of a photoelectron and its binding energy inside the solid, i.e. defines PES as a chemical sensitive technique, and ii) an immediate and straightforward definition of initial and final states of the photoemission process, contrasted with other techniques able to probe the electronic structure of matter using indirect or mixed measures of electronic states, like optical techniques. Historically, PES was widely applied to molecules in the gas phase, but in the present contribution we will focus on solids, in particular quantum materials. ${ }^{26}$ 
A typical experimental set up and schematic energy level diagram are shown in Figure 1(a), along with a PES spectrum of $\mathrm{La}_{0.67} \mathrm{Sr}_{0.33} \mathrm{MnO}_{3}$ (Figure 1(c)), where one notices sharp peaks superimposed on a background of secondary electrons. Well-defined peaks, the so-called primary electron peaks, correspond to core level lines from deep electronic states (different symmetries of the corresponding electronic orbitals are labeled in Figure1(c)); the background of secondary electrons corresponds to electrons having 'lost' information via multiple scattering on their travel through the solid. Information about the electronic, structural and magnetic properties of the ground state of a solid is obtained by analysis of core level structure, including intensities, energy shifts and satellite structure associated with local or collective excitations. Variations with parameteres such as temperature, light polarisation and electric or magnetic fields are also important and all may be compared with theoeretical calculations. Furthermore, angle scanned experiments of core level peaks from the basis of X-ray photoelectron diffraction spectroscopy, where important structural information can be acquired. ${ }^{12}$ Core level PES, with both acronyms XPS and ESCA defined above, has been enormously successful in disentangling oxidation states in , for example, mixed-valence compounds and bonding character (covalency and/or ionicity) of complex systems and of heterostructures ${ }^{11-13,27}$

\subsection{Description and modelling of the ARPES process}

On the high kinetic energy (low binding energy) side of the spectrum in Figure 1(c), the valence band contains information on the extended states in the solid. By measuring the intensity of photoelectrons as a function of their kinetic energy and emission angle, ARPES gives access to the electron-removal spectral function, naturally defined in the crystal momen-

tum space. From the theoretical point of view, excellent reviews are already available, ${ }^{13,14}$ and so we will only briefly recall here the main steps of the ARPES process, and how they are usually modeled. The photoemission can be described as an optical transition from an N-electron state to an ionized (N-1)-electron state (both characterized by many-body 
wave-functions with appropriate boundary conditions at the surface) and including a propagating plane-wave in vacuum (with a finite amplitude in the crystal, therefore providing some overlap with the initial state). This so-called "one-step model" (which includes scattering processes subsequent to the photoemission event) is quite complex from both the formalism and the simulation point of view, so it will not be discussed in detail here (we refer the interested reader to Ref. ${ }^{28}$ ). Rather, we recall a simpler and widely adopted scheme, i.e. the so-called "three-step model", in which the process is subdivided in three steps (Figure2):

i) Optical excitation, which includes all the information on the material's underlying electronic structure; this can be described as a vertical transition in the reduced zone scheme $\left(\mathbf{k}_{f}-\mathbf{k}_{i}=0\right)$ or, equivalently, by involving a reciprocal-lattice vector $\mathbf{G}$ in the extended zone scheme $\left(\mathbf{k}_{f}-\mathbf{k}_{i}=\mathbf{G}\right)$;

ii) Transport to the surface, which can be modelled as an effective mean free path of an electron that reaches the surface without any scattering event, i.e. conserving energy and momentum;

iii) Transmission through the surface and escape in the vacuum, which depends on the energy of the excited electron and on the material's work function.

In what follows, we will describe in closer detail the first step. The calculation of the transition probability $\omega_{f i}$ for an optical excitation from the N-electron ground-state $\Psi_{i}^{N}$ to one of the possible final states $\Psi_{f}^{N}$ is based on Fermi's golden rule:

$$
\omega_{f i}=\frac{2 \pi}{\hbar}\left|<\Psi_{f}^{N}\right| H_{i n t}\left|\Psi_{i}^{N}>\right|^{2} \delta\left(E_{f}^{N}-E_{i}^{N}-h \nu\right)
$$

where $E_{i}^{N}=E_{i}^{N-1}-E_{B}^{\mathbf{k}}$ and $E_{f}^{N}=E_{f}^{N-1}+E_{k i n}$ denote the N-particle initial and final state energies, respectively, and $E_{B}^{\mathbf{k}}$ is the binding energy of the photoelectron with momentum $\mathbf{k}$ and kinetic energy $E_{k i n}$.

Within the so-called "dipole approximation" (valid for typical photon energies in the ultra-violet range, where the photon wavelength is much larger than atomic dimensions), 


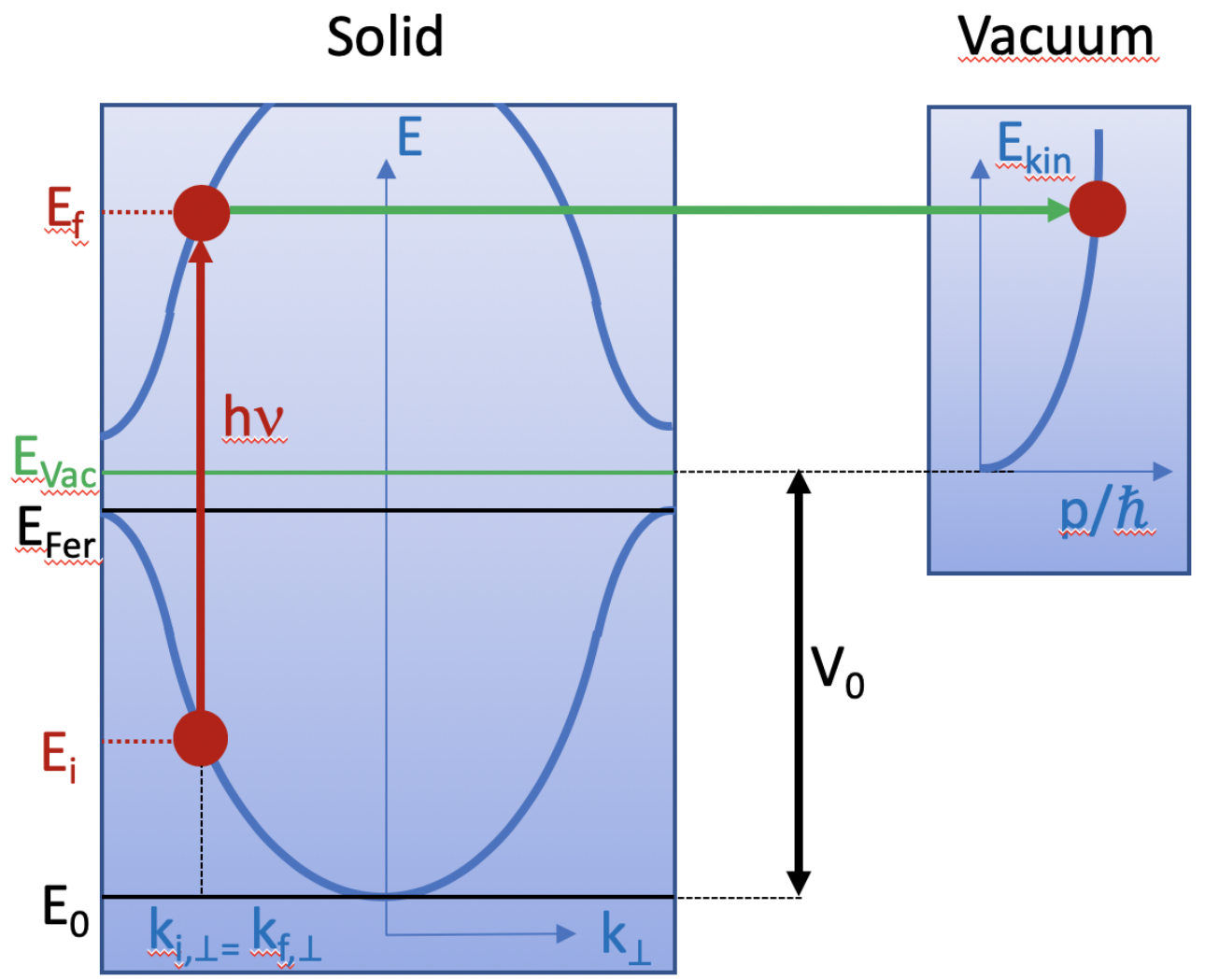

Figure 2: Kinematics of the photoemission process within the three step model: Optical (vertical) transition (left panel), the momentum conservation being possibly assisted by the $\mathbf{G}$ vector in the reduced zone scheme); the photoelectron travels to the surface and the final state in vacuum is assumed to be free-electron-like (right panel). Quantities related to the photoemission energetics are also reported (see text).

the interaction Hamiltonian can be written as:

$$
H_{i n t}=\frac{e}{m c} \mathbf{A} \cdot \mathbf{p}
$$

where $\mathbf{p}$ denotes the electronic momentum operator and $\mathbf{A}$ denotes the electromagnetic vector potential. By further adopting the "sudden approximation" (i.e. implying that the photoemission process is sudden or, in other words, that the electron is instantaneously removed and relaxation processes are neglected), the final state can be factorised into an antisymmetric product of the (single particle) photoelectron state $\phi_{f}$ and the Slater determinant for the (N-1)-particle system, $\Psi_{f}^{N-1}$. In parallel, the initial state can be written as an antisymmetric product of a one-particle state $\phi_{i}^{\mathbf{k}}$ and the antisymmetric state for the 
(N-1)-particle system $\Psi_{i}^{N-1}$. The photoelectron intensity can therefore be written as:

$$
I(\mathbf{k}, E) \propto \sum_{f, i} f(E)\left|M_{f, i}^{\mathbf{k}}\right|^{2} A(\mathbf{k}, E) \delta\left(\mathbf{k}_{\mathbf{i}}-\mathbf{k}_{\mathbf{f}}+\mathbf{G}\right) \delta\left(\varepsilon_{f}+E_{f}^{N-1}+E-E_{I}^{N}-h \nu\right)
$$

where the one-electron transition matrix elements are denoted as $M_{f, i}^{\mathbf{k}} \propto<\phi_{f}^{\mathbf{k}}|\mathbf{A} \cdot \mathbf{p}| \phi_{i}^{\mathbf{k}}>$ and $f(E)$ is the Fermi-Dirac distribution. $A(\mathbf{k}, E)$ is the one-particle spectral function taking into account the excitation spectrum of the (N-1)-electrons and can be expressed as a Lorentzian of the proper self-energy $\Sigma(\mathbf{k}, E)=\Sigma^{\prime}(\mathbf{k}, E)+i \Sigma^{\prime \prime}(\mathbf{k}, E)$

$$
A(\mathbf{k}, E)=-\frac{1}{\pi} \frac{\Sigma^{\prime \prime}(\mathbf{k}, E)}{\left[E-\varepsilon_{i}-\Sigma^{\prime}(\mathbf{k}, E)\right]^{2}+\left[\Sigma^{\prime \prime}(\mathbf{k}, E)\right]^{2}}
$$

For "uncorrelated" systems $(|\Sigma(\mathbf{k}, E)| \rightarrow 0)$, the spectral function reduces to a $\delta$ function in energy and the observed quasiparticle energies coincide with the initial one-electron ground-state $\varepsilon_{i}$. On the other hand, when strong correlations are present, the quasiparticle energy is renormalized and there is an energy broadening (i.e. finite lifetime) due to manybody interactions. The spectral function can therefore be seen, in the first case, as a direct probe for the band-structure and, in the second case, as an investigation tool for correlation effects.

As for step iii) above, we remark that in ARPES the emission direction of the photoelectron is well-defined and its momentum can therefore be derived from the kinetic energy and the emission angle. Furthermore, for photon wave-vectors in the UV-range, one can neglect the momentum transfer from the photons to the photo-electrons. When passing through the surface, the in-plane momentum is conserved and the momentum parallel to the surface can be expressed by:

$$
\mathbf{p}_{\|}=\hbar \mathbf{k}_{\|}=\sqrt{2 m E_{k i n}} \sin \theta
$$

(note that $\mathbf{p}_{\|}$is equal to the parallel component of the electrons within the solid in the extended zone scheme). On the other hand, due to the state's finite extension along the 
surface normal and consequent loss of translational symmetry, the out-of-plane momentum $\mathbf{k}_{\perp}$ is not a good quantum number and the perpendicular component of the crystal momentum is not conserved. Therefore, in order to determine the out-of-plane momentum, one needs additional approximations, such as assuming a free-electron final state with energy dispersion given by:

$$
E_{f}(\mathbf{k})=\frac{\hbar^{2}\left(\mathbf{k}_{\|}^{2}+\mathbf{k}_{\perp}^{2}\right)}{2 m}-E_{0}
$$

where $E_{0}$ denotes the bottom of the valence band (cfr Figure 2). In turn one obtains

$$
\mathbf{p}_{\perp}=\hbar \mathbf{k}_{\perp}=\sqrt{2 m\left(E_{k i n} \cos ^{2} \theta+V_{0}\right)}
$$

where $E_{f}=E_{k i n}+V_{0}, V_{0}=\left|E_{0}\right|+\Phi$ is the inner potential, taking into account the potential step along the surface normal ( $\Phi$ being the work-function). $V_{0}$ can be evaluated by fitting the experimentally measured $\mathbf{k}_{\perp}$ periodicity to the extension of the Brillouin zone in the perpendicular direction.

\subsection{Spectrometers: past and present}

Although the merits of PES and ARPES have been known since the 70s, the power of the ARPES technique and the amount of information derived from photoemission experiments has increased in parallel with improvements in the technical performance of electron spectrometers. The geometry of an ARPES experiment is shown in Figure 3a. Photons are incident on the sample, which is mounted on an in-vacuum goniometer. As evident from Eqns. 1 and 7, both the kinetic energy distribution and the emission angle of the photoemitted electrons must be determined. The former is typically achieved by use of an electrostatic hemispherical analyser, although other designs, such as time-of-flight systems, are popular for certain experiments. Traditionally, a spectrometer with a small angular acceptance would be utilised, and either the sample would be rotated relative to the spectrometer, or the whole spectrometer would be mounted in the sample vacuum chamber and rotated around 

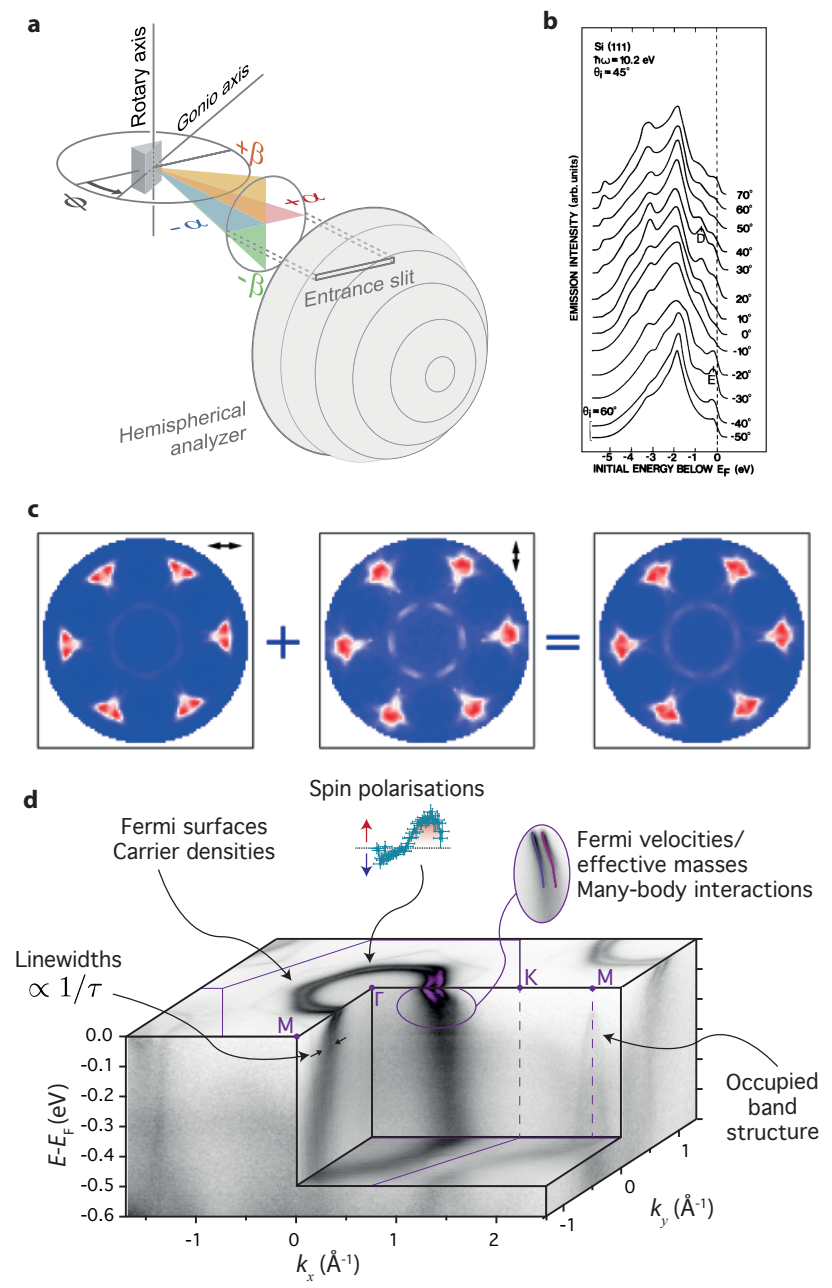

Figure 3: ARPES geometry and example data. (a) Geometry and main experimental parameter varied/scanned in an ARPES experiment. Reproduced with permission from ref. ${ }^{29}$ Copyright 2018 AIP publishing; (b) A collection of angle-resolved spectra measured for different emission angles from a $\mathrm{Si}(111)$ surface. Reprinted with permission from ref. ${ }^{30}$ Copyright 1979 AVS publishing; (c) Fermi surface mapping of Be(0001) single crystal measured in the so called scanned mode, using horizontal and vertical polarization light (as indicated by the arrows). (d) Data "cube" of the low energy electronic structure of $\mathrm{PtCoO}_{2}$, showing the range of electronic structure features that can be accessed from such a data set. Adapted with permission from ref. ${ }^{31}$ Copyright 2017 Springer Nature.

the sample position on its own goniometer.

Rotating the sample or analyser about one axis and measuring a spectrum (intensity vs. kinetic energy at fixed photon energy) for each angular point allows building up an overview of the momentum-dependent dispersions of the band structure of the material along a particular direction in momentum-space (Figure 3b). Remotely controlled scan- 
ning instruments have been developed since the 1990s, with landmark papers by several groups. ${ }^{32-35}$ This enabled a large set of spectra to be taken along a high symmetry direction, which could then be assembled into a so-called carpet allowing better visualisation of the electronic structrure. ${ }^{32,33}$ Alternatively, measuring a high number of angular points (up to 10000) homogeneously distributed over the hemisphere above the sample, by coordinating polar and azimuth movements with precision as high as 0.001 degree, allowed the measurement of a projected Fermi surface with an average acquisition time of 12 hours (see Figure 3c). The late 1980s saw an evolution from single-channel (1D) detectors to so-called 'multidimensional acquisition' modes, where multiple spectra covering a range of emission angles and kinetic energy values can be recorded simultaneously. This capability was widely exploited in the study of high $T_{c}$ superconductors, ${ }^{15}$ and is a core feature of most modern ARPES setups. Typically, a slice of the angular distribution is measured along a direction set by an entrance slit of the hemispherical analyser (Figure 3a). The sample is rotated azimuthally to align the desired direction parallel to the entrance slit in order to measure a dispersion (carpet), while it can be scanned perpendicular to this in order to map the Fermi surface (Figure 3c). Moreover, at large scale facilities one may access full control of light polarization (linear and circular), a feature often exploited to determine the symmetry of the electronic states (see Figure 3c and Annex A). A high-precision sample manipulator, capable of achieving low sample temperatures and operating in ultra-high vacuum, is thus a crucial part of any photoemission setup, ${ }^{36}$ while some spectrometers also allow the possibility to tune the angular acceptance range further using electrostatic deflectors. With such setups, it is now possible to acquire on the order of $10^{8}$ data points in just a few hours, providing a three-dimensional ( $E$ vs. $k_{x}$ vs. $k_{y}$ ) dataset of the electronic structure of a material (Figure 3d). As well as providing a detailed overview of the electronic structure of a material, this enables new quantitative analysis, allowing, for example, extracting Fermi surfaces, carrier densities, and effective masses in good agreement with ultra-precise bulk probes such as de Hass van Alphen measurements, ${ }^{37}$ and performing quantitative analysis 
to extract quasiparticle lifetimes and detailed insights on the many-body interactions at play (see Figure 3d and Section 2.3).

\subsection{Use of light polarization in ARPES: symmetry of electronic} states
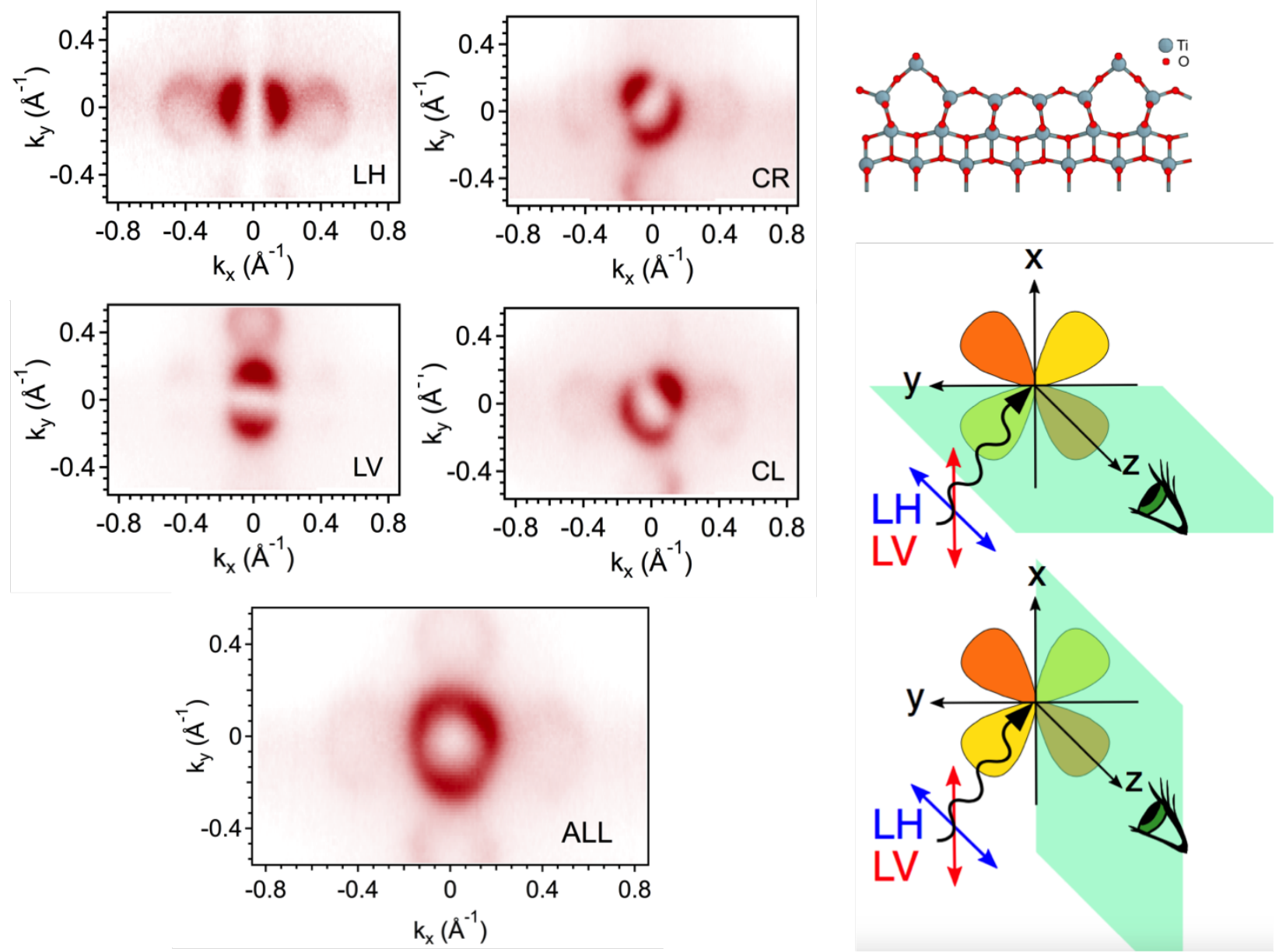

Figure 4: $1^{\text {st }}$ Brillouin Zone of anatase $\mathrm{TiO}_{2}$ Fermi surfaces measured with hv= $46 \mathrm{eV}$ and with all the photon polarisations: LH linear horizontal, LV linear vertical, CR circular right CL circular left. Panel ALL shows the sum of all the four Fermi surfaces, mediating the symmetry-related lack of intensity typical of electronic states with $d_{x y}$ orbital character. Sketch of the geometry of the experiment with a $d_{x y}$ orbital relative to light polarization, and of symmetry selection rules. Both the light polarisation vectors and the orbital possess well-defined parity with respect to the $y z$ and $x z$ mirror planes. Top right: sketch of the $(1 \mathrm{x} 4)(4 \mathrm{x} 1)$ reconstruction at the surface of anatase $\mathrm{TiO}_{2}$. Adapted with permission from Ref $^{38}$ Copyright 2001 American Physical Society and from Ref. ${ }^{39}$ Copyright 2020 American Physical Society. 
The use of polarized light, and the important information one may extract from polarization dependent ARPES, is explained in Figure 4, where a plot in $k_{x}$ and $k_{y}$, cut at the Fermi energy is shown for a thin film of anatase $\mathrm{TiO}_{2}$. This generate a Fermi surface map (see Figure 3a,d). Figure 4 shows the Fermi contours measured in the $1^{\text {st }}$ Brillouin zone while varying the light polarisation at $\mathrm{h} \nu=46 \mathrm{eV}$. The bright circle centred at the $\Gamma$ point corresponds to a parabolic dispersion. In addition, several replica of this Fermi pocket are evident displaced along both the $k_{x}$ and $k_{y}$ directions. In anatase $\mathrm{TiO}_{2}$, a $(4 \times 1)-(1 \times 4)$ reconstruction is observed, ${ }^{39-41}$ and a sketch of this rearrangement of the surface is shown in the figure. The origin of such reconstruction has been proposed, and experimentally confirmed, to arise from a structural reorganisation able to release the large surface stress of the unreconstructed anatase surface. ${ }^{38}$ This observation differs from other transition metal oxide systems that display various surface reconstructions for small changes in the density of the oxygen vacancies. ${ }^{42,43}$

The uneven distribution of the measured PES intensity between different replica bands reflects the weak nature of the periodic potential associated with the $(4 \times 1)-(1 \times 4)$ reconstruction of $T i$ atoms at the anatase(100) surface. ${ }^{38}$ Meanwhile, the angular asymmetry within a pocket is a result of the symmetry of the electronic states, in this case of the $t_{2 g}$ orbitals arising from Ti $3 d$ states close to the Fermi level. This is a result of the one-electron transition matrix element in Eqn. 4. Figure 4 describes the resulting mutual relationship between light polarization, the electronic orbitals and the geometry of the experiment. In particular, symmetry-related selection rules indicate that the 2DEG state of anatase has a $d_{x y}$ orbital character. The sample is probed along the $\Gamma-X$ direction, aligned with the analyser slit. Two orthogonal mirror planes are therefore identified: the $x z$ plane (defined by the analyser slit and the surface normal) and the $y z$ plane, which contains the photon beam and the surface normal. In this geometry, the linear horizontal polarisation (LH) has odd mirror symmetry with respect to the $x z$ plane, whilst the vertical polarisation (LV) has even symmetry. Let us now consider the symmetries of the $t_{2 g}$ orbitals (i.e. $d_{x y}, d_{y z}$ and $d_{x z}$ 
) with respect to such a plane: the $d_{y z}$ has even parity, while both $d_{x y}$ and $d_{x z}$ possess odd symmetry. Thus, from panel $b$ ) of Figure 4 one can readily exclude $d_{y z}$ orbital character, as photoemission intensity for $k_{y}=0 \AA^{-1}$ should appear when excited with LV polarisation (even symmetry of the final state). On the other hand, switching to LH polarisation the spectral intensity vanishes for $k_{x}=0 \stackrel{\circ}{ }^{-1}$ (panel a ). LH has even parity under reflection about the $y z$ plane. The measured photoemitted final state has to be even with respect to both the $x z$ and $y z$ planes, namely it should have $d_{x z}$ orbital character (even symmetry under reflection with respect to $y z$ plane). Thus, the orbital character of the 2DEG is $d_{y z}$, as expected from the degeneracy-lifting of the $t_{2 g}$ caused by the tetragonal unit cell structure

of anatase. This result is consistent with the reported literature both for anatase ${ }^{44-46}$ and for other transition metal oxides. ${ }^{47,48}$

\subsection{Spin-resolved ARPES}

The objective of performing a PES experiment with all the relevant information at hand, the all-in-one experiment, cannot be reached without spin-detection. The spin information is in principle preserved during the photoemission process, and the full vectorial determination of the spin direction in a band, together with the presence or absence of magnetic orders, is important both for fundamental reasons and for future application. In the case of quantum materials, prominent examples are: i) the spin-orbit driven spin-momentum locking of the band structure in topological insulators, a property that cannot be measured without a spinresolved technique, ${ }^{49}$ and ii) the layer-dependent spin-orbital texture of the topological state spanning across a quintuple layer in TIs, producing a complex in-plane and out-of-plane spin texture. ${ }^{50}$ While both high-energy and high-momentum resolution of photoelectrons have become widely available thanks to the advances in electrostatic analyzers, as described in the previous section, the full vectorial determination of the spin of the photoelectrons has been limited for decades by poor statistics. For example, spin discrimination based on spin-orbit (Mott-) scattering suffers from a the low cross-section, resulting in a poor 
figure of merit for spin-resolved analysis (typically $\left.10^{-3} / 10^{-4}\right) .{ }^{51}$ The strong reduction of intensity as compared to a conventional ARPES experiment led to a requirement to use significantly relaxed energy resolution, limiting for many years spin resolved PES experiments to mostly core level lines and/or to valence band studies, not $k$-resolved, of ferromagnetic systems. The explosion of quantum materials research, and in general the interest on systems where the spin texture is not solely driven by purely magnetic interaction, as the case of Rashba and topological systems, revamped the technological development related to spinARPES. Optimized Mott-scattering systems and spin-polarised electron diffraction setups were developed, ${ }^{52,53}$ and low energy scattering on ferromagnetic targets (V-LEED) have successfully demonstrated an intensity gain of several order of magnitudes, making possible data acquisition of adequate statistics with comparable energy and momentum resolution with respect to the non-spin-resolved spectra. ${ }^{54-58}$ Recently, multidimensional spin-ARPES has been demonstrated, where imaging techniques combined with multichannel intensity detection in a time of flight detector allows one to measure 4D arrays of data. ${ }^{59}$

\section{Comparison between ARPES and density functional theory}

First-principles approaches, such as Density Functional Theory (DFT) ${ }^{61-65}$ represent one of the most widely adopted methodologies to explore materials' band structures from a theoretical perspective. In a nutshell, DFT simplifies the (otherwise unsolvable) many-electron problem into a set of manageable independent-particle equations, the so called Kohn-Sham equations $\left(\mathrm{see}^{62}\right.$ ), and allows the ab-initio calculation of a material band-structure. However, the comparison between ARPES and DFT shows many different challenges, as schematically represented in Figure 5. On the experimental side, intrinsic limits exist, related to the apparatus resolution and the sample quality. Furthermore, as discussed above, commonly adopted models for ARPES imply several different approximations (sudden approximation, three-step 


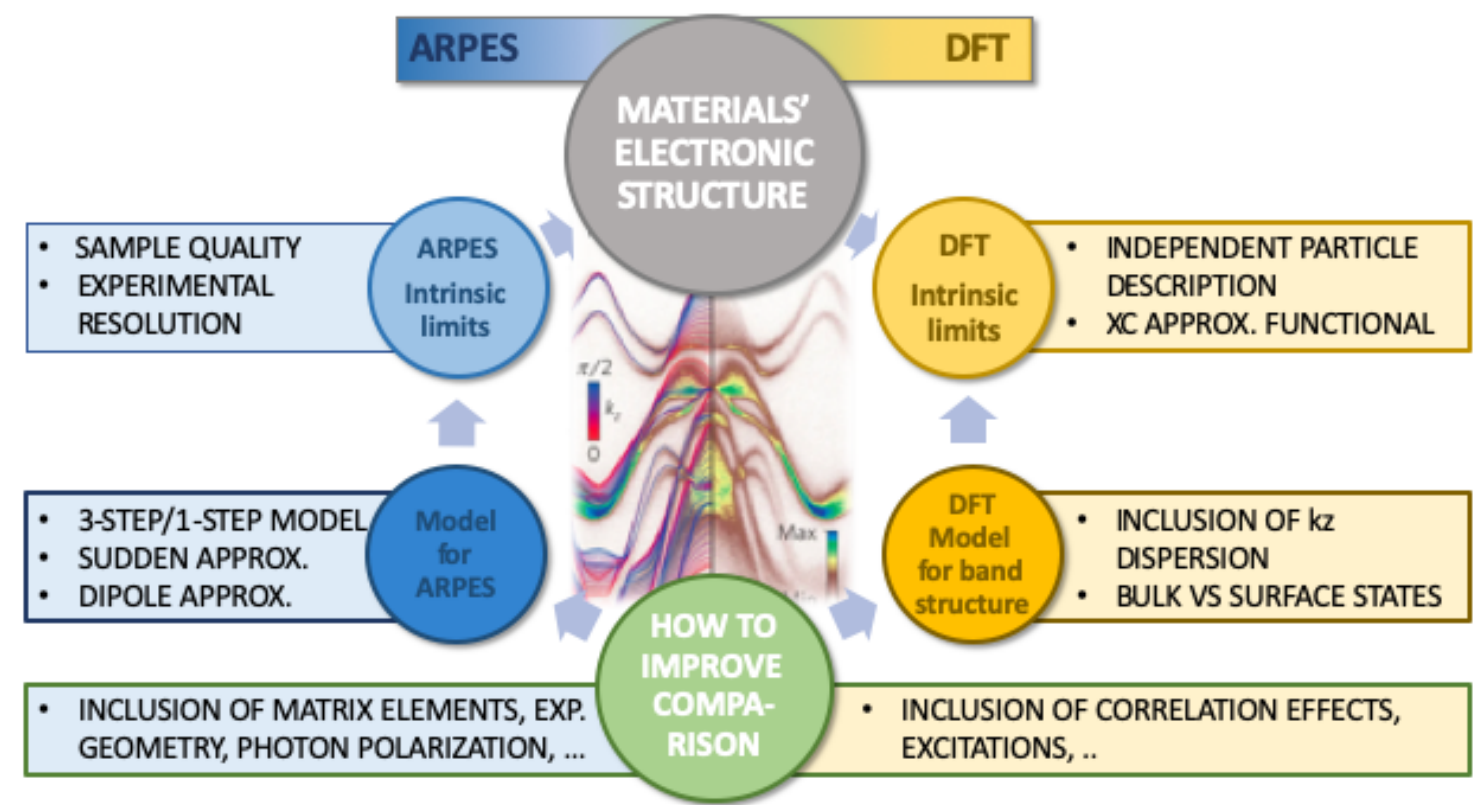

Figure 5: Comparison between ARPES and DFT: Strengths and limitations. Central band-structure adapted with permission from Ref. ${ }^{60}$ Copyright 2014 Springer Nature.

model, etc). In this respect, we remark that recent theoretical developments concern the explicit evaluation of matrix elements (often assumed to be otherwise constant or interpreted in terms of the simple symmetry-based selection rules discussed above) and their explicit dependence on the photon polarization, resulting in important modulation of the theoretical spectra. ${ }^{66}$ We also note that Moser in Ref. ${ }^{67}$ proposed an expression of the ARPES spectral weight of a given Bloch band as determined by the Fourier transform of its associated Wannier function (therefore providing information on the electronic wave function, including local orbital symmetry) multiplied by a polarization-dependent factor, enforcing the dipole selection rules (giving rise to geometric effects, such as circular dichroism). Although the formalism used by Moser is based on the three-step model and on a single-particle picture, the proposed photoemission intensity was found to work well in many different systems (including graphene, iridates and other complex oxides).

On the $a b$-initio side, we recall $\left(\mathrm{see}^{61-65}\right.$ ) that DFT is a single-particle ground-state 
theory and requires approximations for the exchange-correlation potential, which, depending on the system, may be valid or not. For example, in materials with relevant many-body effects and strong electronic correlations, DFT may result in an inaccurate description of the band structure. Furthermore, being a ground-state theory, DFT cannot rigorously describe excitations. In this respect, since ARPES probes occupied electronic states, well-known DFT failures concerning, for example, the energy-gap and excited electronic states do not result in dramatic effects when comparing DFT results and photoemission data. The effects of banddispersion in the k-space direction corresponding to that perpendicular to the sample surface in real space can be well explored in ARPES by changing the photon energy (see Eq. 8) and can be compared to the corresponding calculated band structures. An example of the latter (sometimes referred to as "projected band structure", i.e. ideally projected on the surface plane) is reported in Figure 6 a) (for convenience, the specific case of $\mathrm{IrO}_{2}{ }^{68}$ is reported here, though all the following arguments remain clearly valid for any material). Stronger signals are obtained in the case when bands do not disperse much in the $k_{\perp}$ direction. This behaviour is also reflected in the spectral function (cfr Eq. 5), reported in Figure 6 b), which allows for an easier comparison with ARPES spectra. A further development is the explicit DFT simulation of surfaces (both in terms of band structures and of spectral functions for semi-infinite slabs $\left.{ }^{69,70}\right)$. In the simulated results, one can easily identify the surface states, since they often appear in energy regions which are otherwise forbidden in the corresponding bulk. Their surface character can be further explored by expanding the electronic states as a linear combination of atomic orbitals and calculating the weight that these states show on different atoms. For surface states, a large weight on the surface atoms is obtained. The bulk-vs-surface character of different electronic states can be cross-checked in ARPES, by exploring the dispersion as a function of photon energy, the changes being rather small (large) for localized surface (dispersive bulk) states. 

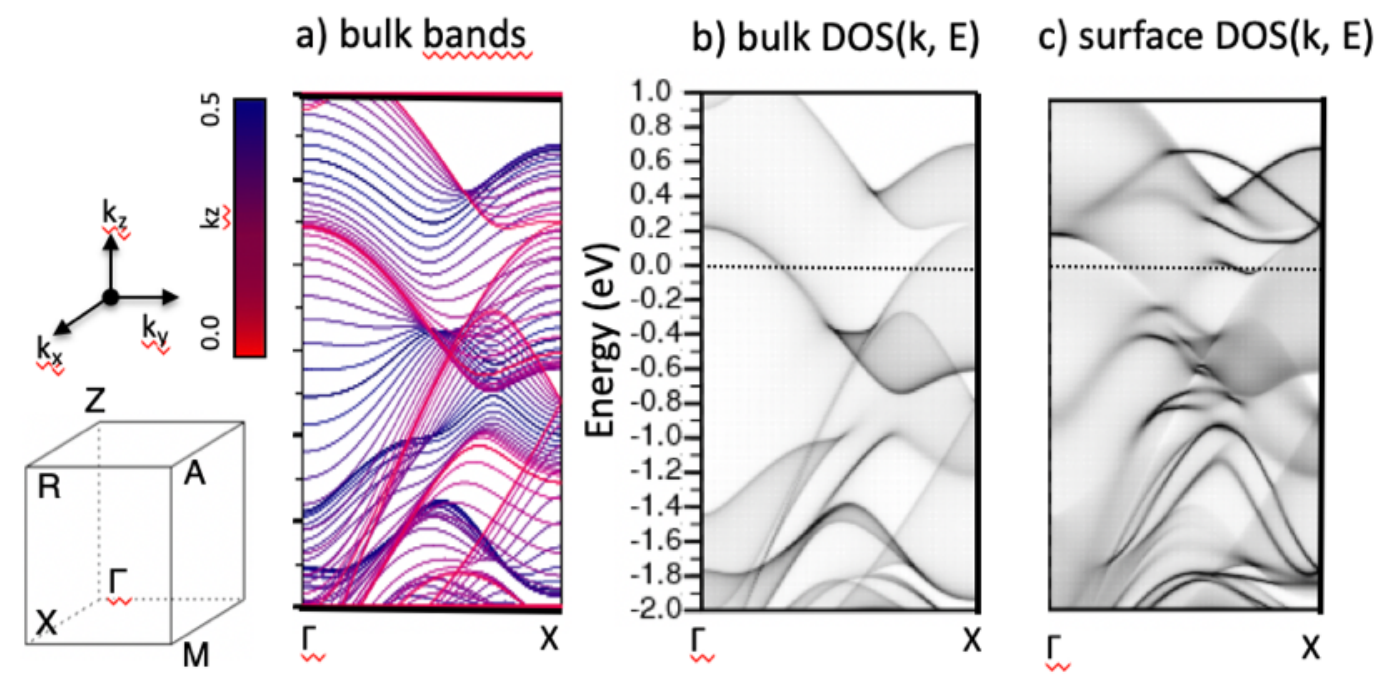

Figure 6: Example of DFT calculations to be compared with ARPES: the case of $\operatorname{IrO}_{2}(110)$ surfaces. a) Bulk band structure along directions parallel to $\Gamma-\mathrm{X}$ and corresponding to different $\mathbf{k}_{z}$ (i.e. different points along $\Gamma$-Z, cfr inset of the Brillouin zone in lower left part). b) Bulk spectral function; c) Surface spectral function. Panels b) and c) adapted with permission from Ref. ${ }^{68}$ Copyright 2018 American Physical Society.

\section{Depth-dependent information in photoemission}

\subsection{Bulk sensitivity and HAXPES}

An important experimental aspect of PES concerns its probing depth. As considered in step 2 of the photoemission process in Section 2.3, we are typically interested in the signal from photoexcited electrons which have traveled to the surface without undergoing scattering events. For valence band photoemission using, for instance, ultra-violet light, the corresponding electron inelastic mean free path is between $5 \AA$ and $10 \AA$. This makes the technique extremely surface sensitive. This surface sensitivity is of utmost importance for studying few-layer systems and, in general, for surface and interface effects, with some selected examples reported in the following sections. Care must, however, be taken when interpreting photoemission data to consider its surface sensitivity. In some strongly correlated materials, for example, a larger electron correlation has been observed in the bulk as compared to the surface, in contrast to what one would expect from simple coordination 
arguments. ${ }^{71-76}$ It is important to emphasise that the problem of surface sensitivity cannot be solved even by having the sample as a single crystal with a perfect surface termination. The surface will always have an electronic structure different from that of the bulk, and this is especially true for strongly correlated systems since small variations of the coordination of atoms with localised $3 d, 4 f$ or $5 f$ valence orbitals will already have a big impact on their narrow bands: a different Madelung potential or a different hybridization strength due to the reduced coordination at the surface results in a vastly different electronic structure. Moreover, at the surface the orbital occupation of the $3 d$ shell in early transition oxides is altered, with the major consequence that the surface may induce insulating behavior in marginally metallic materials, or allow the formation of metallic surfaces on insulating solids. These effects are well-documented in perovskites, where in addition electrostatic instability associated with polar surfaces may lead to major atomic or electronic reconstruction, or possibly both. ${ }^{31,71,72}$ Finally, the fact that, due to the surface sensitivity of the technique, $\mathbf{k}_{\perp}$ is not a good quantum number (see Section 2.3), photoemission intrinsically has a poor resolution in the out-of-plane direction. For two-dimensional electronic states, this does not cause a problem. However, for three-dimensional states, which disperse in the out-of-plane direction, this leads to a significant broadening of the measured spectral features, which will be seen further below.

The use of synchrotron radiation allows the photon energy, and hence the kinetic energy of outgoing photoelectrons, to be varied. This in turn allows the surface sensitivity in an experiment to be tuned. The inelastic mean free paths of the photoexcited electrons are largely thought to follow a so-called "universal curve" (see Figure 1). For a comprehensive review of the different definitions of escape depth and depth information in photoemission, we draw the readers attention to the landmark papers from Tanuma and Powell. ${ }^{77,78}$ In particular, the semi-empirical model by Tanuma, Powell and Penn (TPP-model) is an accurate way to estimate the inelastic mean free path of photoelectrons. ${ }^{77,79}$ When the incident photons excite electrons to kinetic energies close to the minimum in the universal curve (in 
the range between $10 \mathrm{eV}$ and $500 \mathrm{eV}$ ), maximal surface sensitivity is achieved with effective probing depths on the order of only $10 \AA$. To perform an experiment that provides bulk sensitive information, the electron kinetic energies must be either well below or well above the minimum shown in Figure 7). According to experiments and calculations the probing depth in PES experiments increases dramatically at very low electrons energy, and in recent years, laser-based PES experiments in this energy range $(h \nu$ below $7 \mathrm{eV})$ demonstrate submeV energy resolution, access to high angular resolution and a probing depth well above the nanometer range. ${ }^{80,81}$ For low kinetic energies, however, the probing depth is strongly dependent both upon the material and the proper selection of the excitation energy due to final state effects; ${ }^{82}$ moreover, core level lines are not accessible. The high brilliance of last generation synchrotron radiation beamlines made possible in recent years to explore the high energy side of the curve in Figure 7. Increasing the photon energy invariably reduces the photoelectron intensity due to reductions of the cross section in the photoemission process, implying that experiments on the high energy side of the universal curve are always 'photon hungry'. 76

Paying the price of both a limited energy resolution (on the order of 50-100 meV) and the loss of k-resolved information, electron kinetic energies above $3 \mathrm{keV}$ could be explored via hard x-ray PES (HAXPES), ${ }^{71}$ featuring a probing depth up to $20 \mathrm{~nm}$, i.e. much higher bulk sensitivity and access to buried layers for practically any heterostructure or device. ${ }^{83}$ The pre-war experiments conducted by Robinson typically used hard transition metal $\mathrm{K} \alpha$ excitation, as did early experiments from Siegbahn's group. However, the modern era in HAXPES began with Pianetta and Lindau in $1974,{ }^{84}$ and this field has been revived in the last two decades by exploiting the high flux (up to $10^{13}$ photons/second at the sample) available at synchrotrons sources. ${ }^{71}$ Looking at the valence band, the experimental density of states (DOS) measured in the HAXPES regime can be reliably compared to those generated by a variety of calculation methods, and provides relevant information about the difference between bulk and surface electronic properties. ${ }^{11}$ Since the surface contribution to the total 


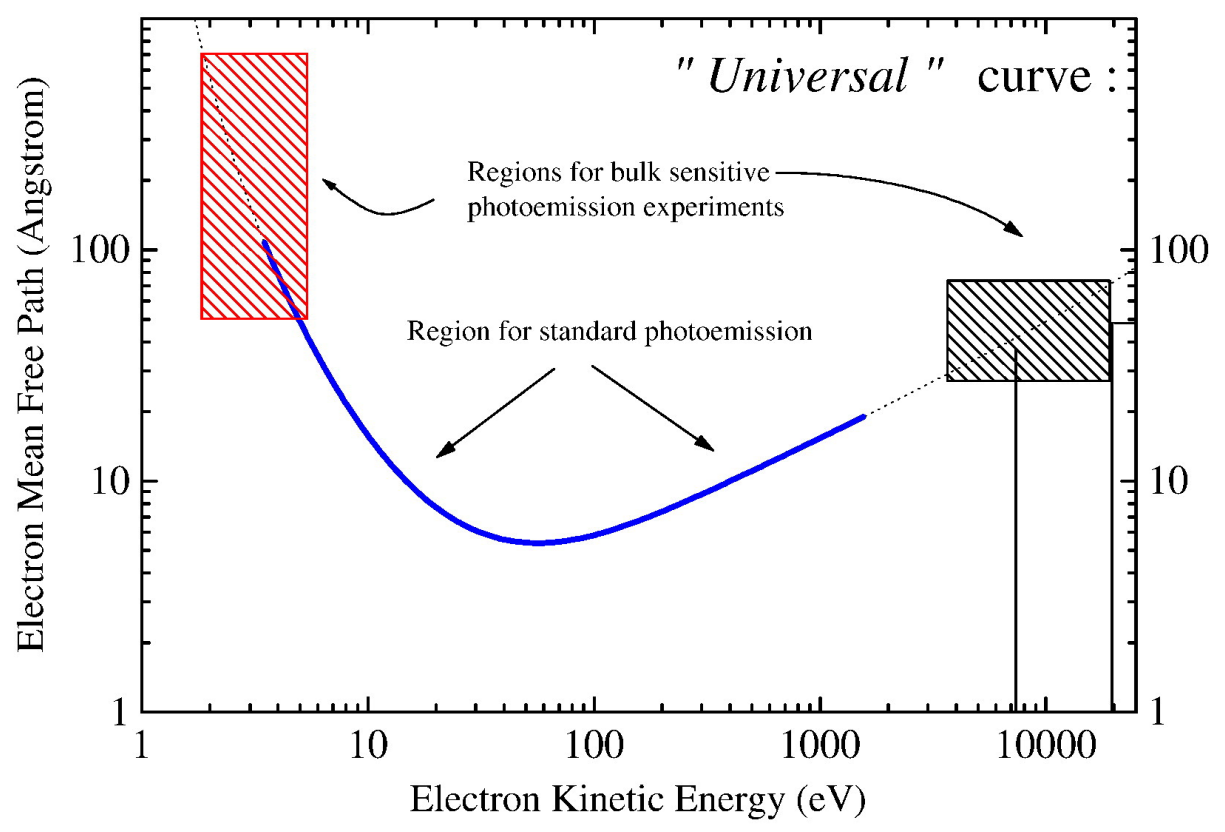

Figure 7: The so-called universal curve of photoelectron mean free path in a solid vs. their kinetic energy, extracted from ref; ${ }^{77}$ the two highlighted zones show where bulk information could be obtained. the zone at high kinetic energy, i.e. above $3 \mathrm{keV}$, correspond to the HAXPES regime;reproduced with permission from ref. ${ }^{76}$ Copyright 2012 Elsevier

photoelectron flux in a HAXPES experiment is typically $<5 \%$ of the total PES signal, ${ }^{85}$ valence band HAXPES reveals valuable information in particular when applied to strongly correlated systems, where uncertainties due to surface preparation and/or enrichment or depletion of oxygen at the surface, hence change of the valency, have been a strong limitation to a detailed comprehension of correlated oxides. ${ }^{71-76}$

\subsection{Use of cross sections in HAXPES}

A further important aspect of HAXPES is that the pattern of relative photoionization cross sections differs markedly from those found at lower photon energy. Extensive calculations for most elements of the periodic table have revealed that all photoionization cross sections decrease with increasing photon energy above $1000 \mathrm{eV}$, but the variation is not the same for all orbitals. ${ }^{88-90}$ The cross sections depend on matrix elements between the final state wavefunction and that for the initial state. Final state wavefunctions are basically free- 

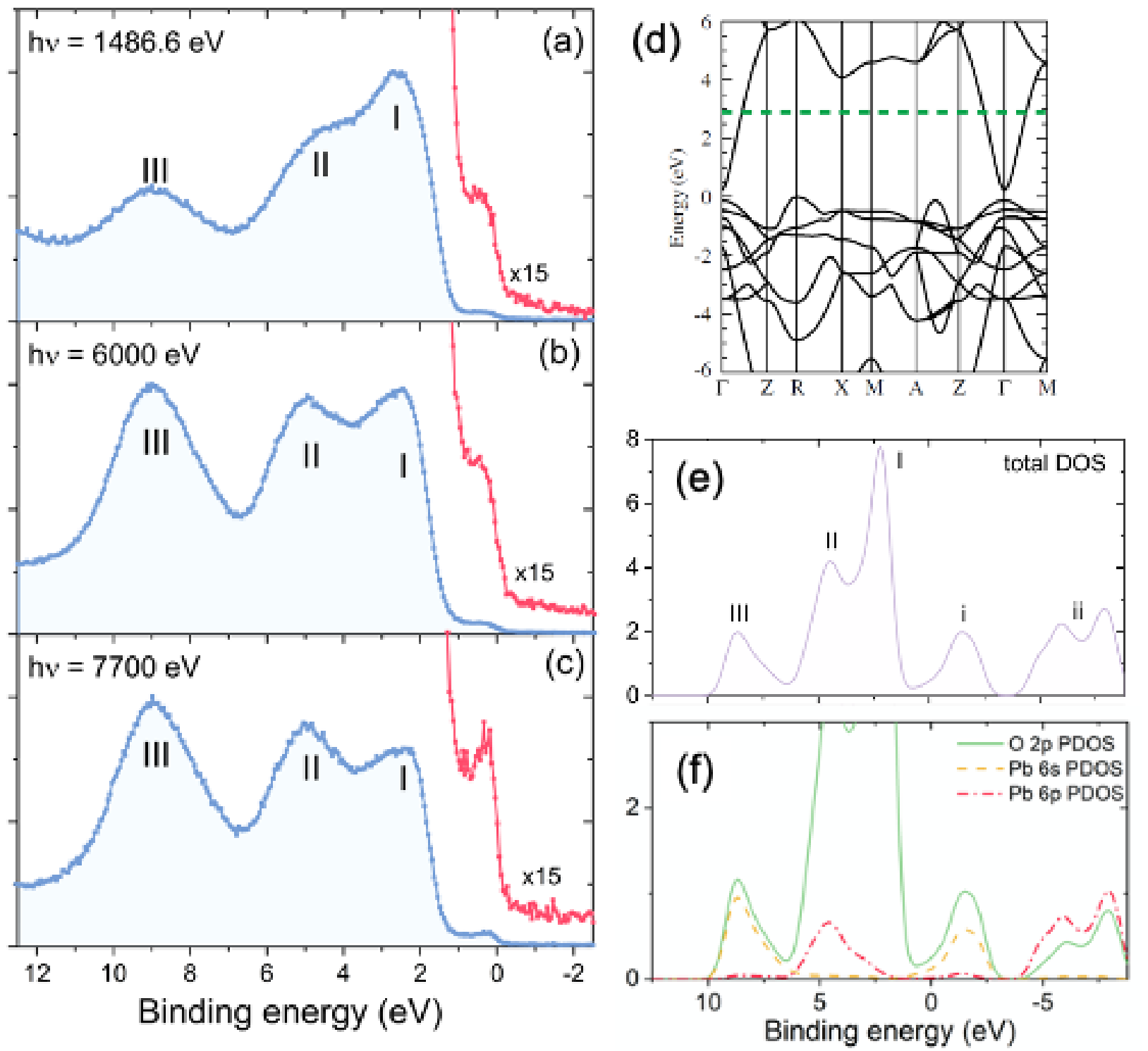

Figure 8: Panel (a),(b),(c):Valence band photoemission spectra of $\mathrm{PbO}_{2}$ measured at different photon energies; the increase of the feature close to the Fermi energy when entering the HAXPES regime is highlighted (red points). (d) The bandstructure of $\mathrm{PbO}_{2}$ calculated with an HSE06 hybrid functional. The position of the charge neutrality level is indicated by the dashed green line. Adapted with permission from ref. ${ }^{86}$ Copyright 2011 American Physical Society. (e) and (f): Calculation of full and partial density of states for $\mathrm{PbO}_{2}$. panels (a),(b),(c),(e) and (f); adapted with permission from ref, ${ }^{87}$ Copyright 2007 American Physical Society.

electron waves, perturbed by the atomic potential of the ionized atom. As the photoelectron kinetic energy increases and the free-electron wavelength decreases, the dipole intensity is 
quickly exhausted when the initial state wavefunction shows smooth variation, as for $O 2 p$, transition metal $3 d$ or lanthanide $4 f$ orbitals. By contrast orbitals with a high principal quantum number $n$ and low angular momentum $l$ have a number $n$-1- $l$ of radial nodes: the short period oscillation of the radial wavefunction close to the nucleus ensures that the dipole intensity drops off much less quickly with increasing photon energy than for orbitals with no radial nodes. ${ }^{11,88-90}$ These effects are nicely illustrated by valence band HAXPES of $\mathrm{PbO}_{2} .{ }^{91}$ This is a highly covalent compound in which both the conduction band ( (identified as i in the DFT DOS in Figure8e) and states at the bottom of the valence band ((identified as III in the same panel e) have significant $\mathrm{Pb} 6 s$ character). It is seen that both of these features increase in intensity relative to band II - which relates to states of more pure $\mathrm{O}$ $2 p$ character - in the experimental spectra on increasing the photon energy (the partially occupied conduction band has been subject to a x15 expansion in the experimental spectra). Moreover these spectra explain why $\mathrm{PbO}_{2}$ is a metallic material. The issue is whether the conduction band crosses the top of the oxygen $2 p$ valence band or if there is a small but well-defined bandgap and conduction band states are occupied due to electrons donated by oxygen vacancies. The HAXPES measurements are clearcut in demonstrating that a partially occupied conduction band sits above the main oxygen $2 p$ valence band, in agreement with both hybrid DFT calculations and neutron diffraction measurements which reveal a high level of oxygen deficiency. ${ }^{86}$ These conclusions are however at variance with more recent theoretical results that suggest that there is indeed band crossing and that $\mathrm{PbO}_{2}$ can be regarded as a 'three dimensional Dirac semi-metal'. ${ }^{92,93}$ However, there are no experiments to support this viewpoint.

\subsection{Angular resolved HAXPES}

Although the extension of valence band HAXPES to the angular resolved mode (a technique termed HARPES) is conceptually simple, a number of technical challenges and physical effects must be taken into account when increasing the kinetic energy of the photoelectrons. 
a
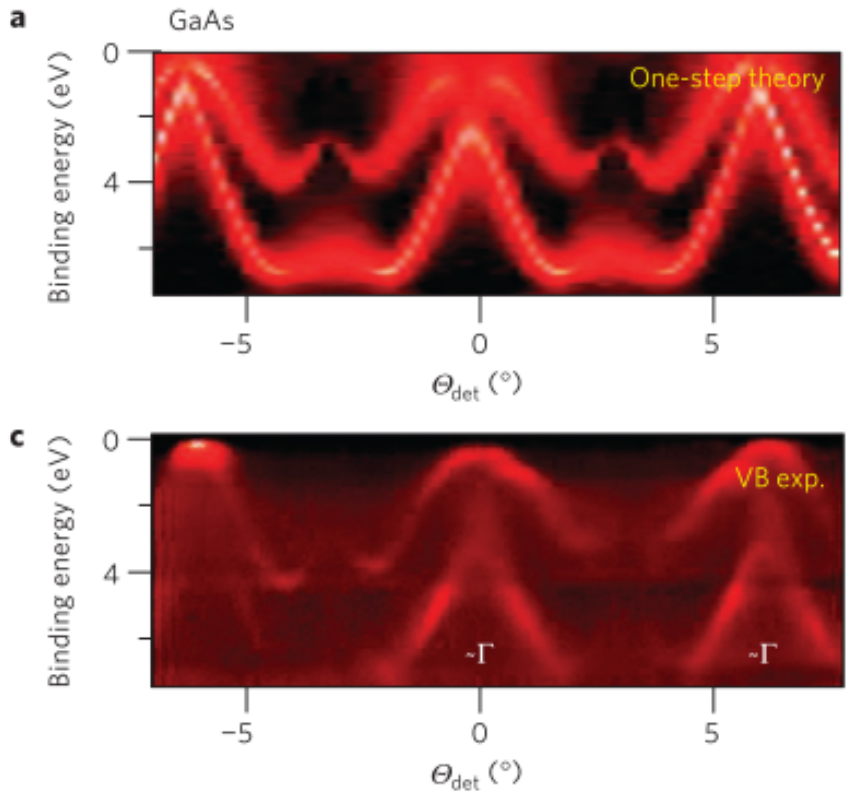

b

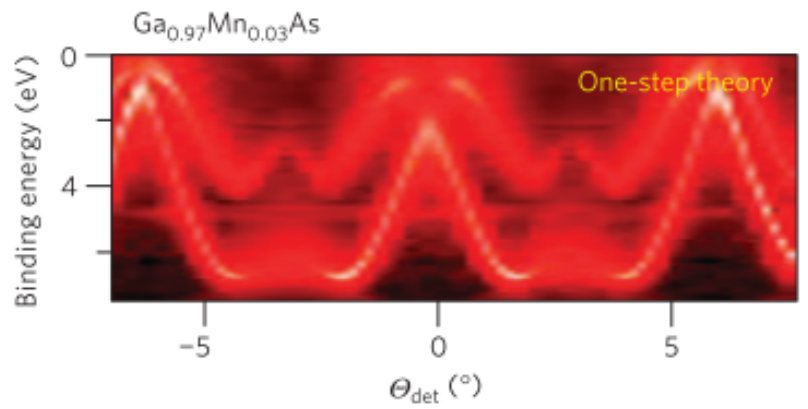

d

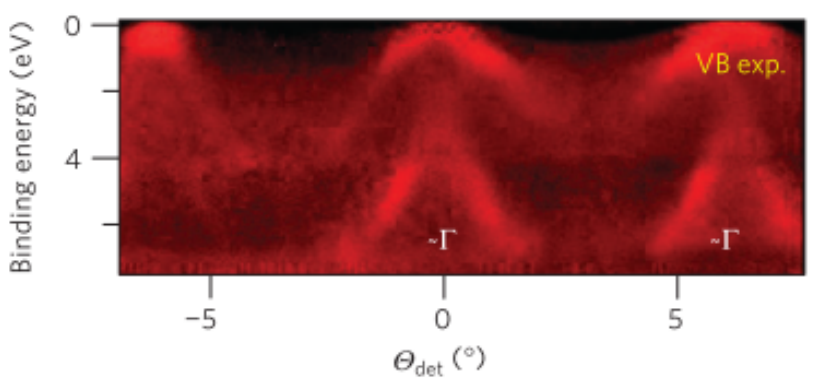

Figure 9: HARPES results from GaAs (left) and 3\% Mn doped GaAs (right). EDC maps are plotted vs. $\Theta$ angle of the analyser detector, $\Theta_{\text {det }}$. Top panels $(\boldsymbol{a}, \boldsymbol{b})$ fully relativistic one-step theory calculation, including matrix element effects. Bottom panels $(\boldsymbol{c}, \boldsymbol{d})$. Experimental HARPES valence band (VB) mapping, measured at 20 Kelvin and at $3.2 \mathrm{keV}$ of photon energy. Smearing of the band features in the Mn doped GaAs results is clearly observed, as due to a modified long-range translational order induced by the Mn impurities. Adapted with permission from ref $\operatorname{Ref}^{94}$ Copyright 2012 Springer Nature.

Some (often important) limitations with respect to the powerful achievements of low energy ARPES are unavoidable, such as: (i) loss of angular resolution, due to the increase of the wave vector $k_{F}$ of the electron momentum in the final state, with a consequent Brillouin zone broadening, (ii) the effect of phonon creation and annihilation related to Debye-Waller effects, that is again a source of broadening in photoemission spectra, (iii) an observed shift of the photoemission lines as due to the 'recoil' effect arising from momentum conservation, an effect particularly relevant in light elements. A detailed description of these effects is found in refs. ${ }^{94-96}$ Despite these limitations, when care is taken to account for and/or minimise these effects, HARPES can still provide direct measurements of the $k$-resolved electronic band structures of solids (Figure 9). Such measurements prove to be particularly useful in the analysis of systems where standard sputtering/annealing surface-preparation procedures destroy structural periodicity, or when chemical etching affects the stoichiometry, 
and in general where the composition and structure of the first layers are not representative of the bulk properties, as often happens in strongly correlated materials, buried/capped heterostructures, and systems doped above the solubility limit, where the distribution of dopants is uneven at the surface. ${ }^{11,76}$

A clear example of the application of HARPES comes from results obtained in the diluted ferromagnetic semiconductor (Ga,Mn)As, shown in Figure 9. HARPES band mapping from non-doped GaAs and 3\% Mn-doped GaAs are compared to one-step fully relativistic calculation including matrix-element effects, normalised to minimize X-ray Photoelectron Diffraction effects. ${ }^{94}$ Panels c,d in Figure 9 show how a single HARPES detector image encompasses several cross-sections of the Brillouin zone, namely three times repeated across $\Gamma-\mathrm{K} / \mathrm{U}-\mathrm{X}-\mathrm{K} / \mathrm{U}-\Gamma$. Comparison between experimental and theoretical results in Figure 9 confirm that the smearing of the band features in Mn-doped GaAs has its origin in Mn impurities, following modification of the long range translational order, and allows a study of the hybridization effects between Mn and the host GaAs. The origin of Mn-induced ferromagnetism in (Ga,Mn)As has been long debated with two opposite mechanisms: hole doping and $p$ - $d$ exchange model on one side, corresponding to strong hybridization on $\mathrm{Mn}$ in the host GaAs band, and the so called impurity model on the other side, where Mn induced states, well separated in energy, form above the valence band maximum leading to doubleexchange ferromagnetism. ${ }^{97}$ Further analysis suggest that the Mn-derived impurity band is merged with the GaAs valence band, thus reconciling the two interpretations, and showing that both the mechanism are active. ${ }^{94,95,98}$

Such an approach has subsequently been applied to other materials, exploiting the fact that information depths as large as $4 \mathrm{~nm}$ with a chemical sensitivity down to $2 \%$ can be achieved, a sensitivity that promises a large number of applications. ${ }^{99-101}$ It is important to underline that phonon smearing will always be present to some degree, implying that lower photon energies should be preferred for high resolution ARPES analysis as is the case for the results shown below. It should be mentioned, however, that the extension to the high 
kinetic energy region of ARPES will increase the diversity brought from ARPES to the tools for studying the electronic structure of solids.

\section{$5 \quad$ Example materials systems}

With the aforementioned capability to probe all the relevant quantum degrees of freedom of the solid, and in particular with its ability to directly probe the momentum-resolved electronic structure and many-body interactions in solids, ARPES has become established as a key probe in the study of quantum materials. We discuss in this section several characteristic examples from materials families and systems that have been particularly intensely studied in recent years. We note that this is not intended as a comprehensive review into the materials physics and chemistry of these systems, but rather as a representative viewpoint of the types of measurements that have proved valuable in the study of such materials, and of the forms of insights that can be gained from these.

\subsection{Spin-orbit materials: non-trivial topology and Rashba effects}

During the last decade, spin-orbit coupling (SOC) has played an increasingly crucial role in condensed matter physics, thanks to its relevance as a rich microscopic mechanism from the fundamental point of view and as a driving force for innovative spintronic applications on the technological side. The SOC, a relativistic "atomic-like" interaction, links the orbital angular momentum $\mathbf{l}$ and the spin angular momentum s, i.e. $H_{s o c} \propto \frac{1}{r} \frac{d V}{d r} \mathbf{l} \cdot \mathbf{s}$ (where $V$ labels the potential and $\frac{d V}{d r}$ its radial derivative).

The spin-orbit interaction is relevant for many phenomena, both in the realm of magnetic and non-magnetic materials. For the sake of brevity, we will touch here only on two classes of compounds where ARPES has been crucial in determining their properties: topological insulators (TI) and "bulk" Rashba materials. Both classes show emergent phenomena, where common probes based on transport give much less direct evidence compared to 
surface-sensitive spin- and angle-resolved photoemission spectroscopy. While many reviews are available in the literature, focused on topological insulators ${ }^{102-104}$ probed by ARPES, ${ }^{105}$ we will limit ourselves to recalling the most basic features of TI and the first pioneering ARPES results on this class of materials. ${ }^{49,106,107}$ Similarly, we will review "bulk" Rashba materials only in terms of the first photoelectron spectroscopy measurements published in the literature.

\subsubsection{Topological insulators}

Topological insulators (TI $)^{102,103}$ in three dimensions are insulators in their bulk phase, though hosting metallic surface states (SS), labelled as "Dirac cones". The SS are protected by the non-trivial topology of the bulk electronic wave functions, whose connection to the SS is often referred to as "bulk-boundary correspondence". As long as the topological protection holds, the metallic surface states will never disappear; their robustness can therefore be exploited in spintronic devices, in addition to being a characteristic feature of a novel state of quantum matter. Indeed, TI are part of the so-called "Dirac materials", i.e. compounds where the relativistic SOC is so relevant that the Dirac equation is the appropriate one to be solved and to describe the electronic structure of the system. Dirac materials constitute an ever-expanding class of compounds, including, among others, Weyl semimetals, ${ }^{108}$ Dirac semimetals, ${ }^{109,110}$ crystalline topological insulators, ${ }^{111,112}$ etc.

In was theoretically predicted ${ }^{113,114}$ in TI that a large SOC drives the "inversion" of bulk conduction and valence bands, i.e. around high-symmetry points, bands with orbital character typical of the conduction (valence) states lie below (above) the Fermi level, or equivalently they are occupied (empty). This band inversion was recognized to be key for realizing a strong 3D TI phase, characterized by deriving topological invariants for a timereversal-invariant band insulator. Without going into details on topological invariants (we refer the interested reader to Ref. ${ }^{102,104,115,116}$ for Berry phases/connection, etc), the latter are essentially integrals over the Brillouin zone of quantities related to electronic wave-functions 
and eigenvalues, that are stable with respect to continuous (i.e. adiabatic) changes of certain parameters.

A distinctive feature of TI is the spin-momentum locking of the gapless linearly-dispersing Dirac-cone SS, i.e. the spin-texture winds circularly around a constant-energy contour in momentum space. In other words, the surface of a 3D TI allows the electron to move in any direction, but once the direction of electronic motion is set, then the spin-direction is uniquely fixed (and viceversa). In closer detail, states at the surface of spin-orbit coupled materials can split into singly-degenerate levels, due to surface-induced inversion symmetry breaking. However, as required by Kramers' theorem, the splitting must vanish at the time-reversal invariant momenta (TRIM) in the 2D surface Brillouin Zone. When connecting two TRIM in the same BZ, the Fermi energy inside the bulk gap will cross these single-degenerate SS either an even or an odd number of times, ${ }^{113,114}$ leading to topologically trivial or non-trivial case, respectively. In other words, a non-trivial $Z_{2}$ topological number in a 3D TI requires the surface to show a Fermi surface pocket enclosing TRIM with non-zero $\pi$ geometrical Berry phase (i.e. odd, as opposed to an even multiple of $\pi$ ).

ARPES played a crucial role in breakthrough discoveries in the whole field of Dirac materials, as it allows measurement of the surface state Fermi surface, (i.e. the number of Fermi pockets enclosing the Kramers points) and the related spin-texture. For example, by means of spin-ARPES on TI one can show that the SS carry a geometrical Berry phase with chiral properties, at variance with conventional surface electronic structure of spin-orbit metals (such as gold), where the Berry phase is zero. Indeed, the whole field of TI had a boost since the first pioneering experimental ARPES data became available, ${ }^{49,106,107,117}$ as recalled below. In addition, one can exploit the dependence of the matrix elements on the light helicity in materials with strong SOC to probe the spin-texture. For example, the difference in photoemission intensity between left- and right-circularly polarized light (i.e. at the basis of circular dichroism) allowed one to obtain results in TI consistent with what was theoretically predicted. ${ }^{118}$ 
a)

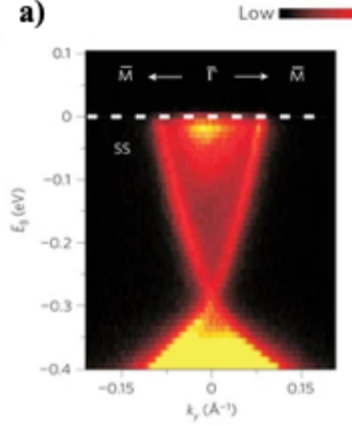

c)

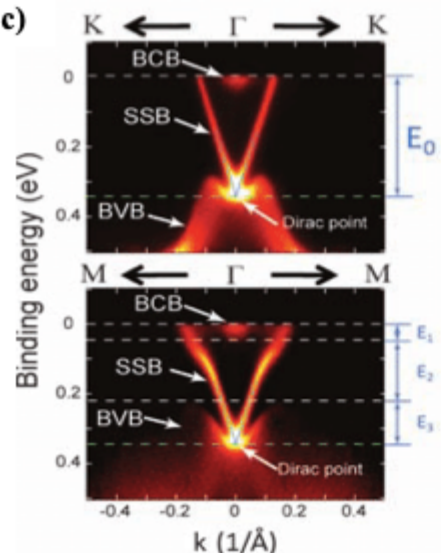

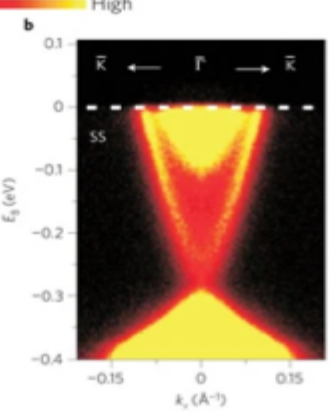

d)

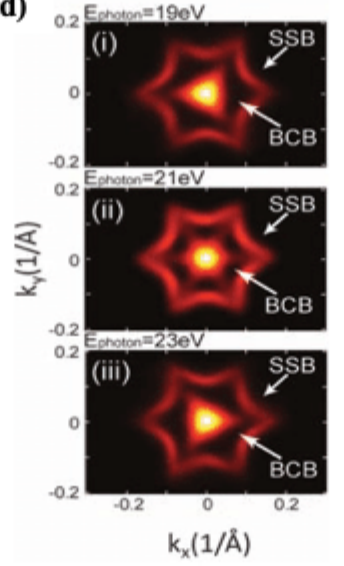

b)

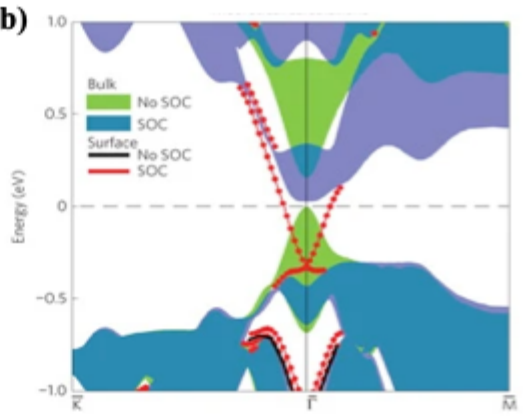

e)

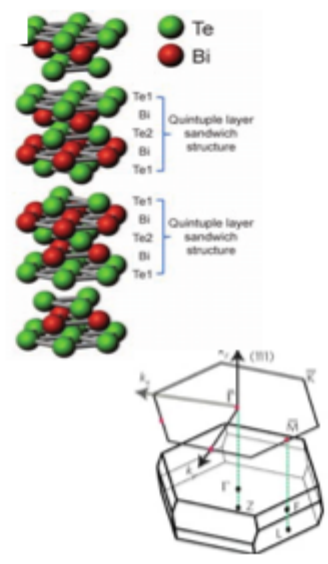

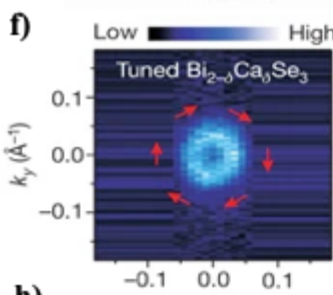

h)

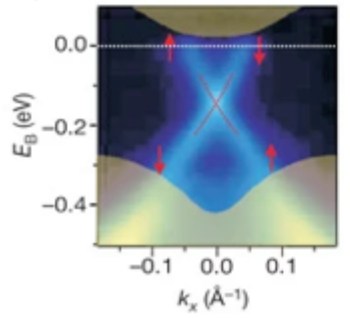

g)

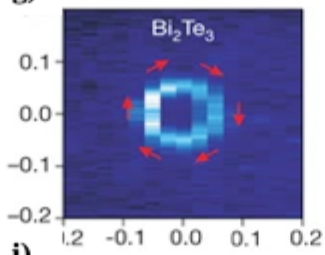

i)

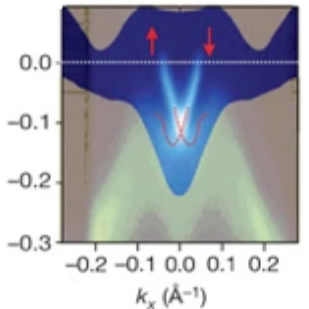

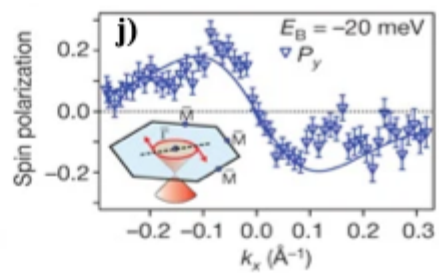

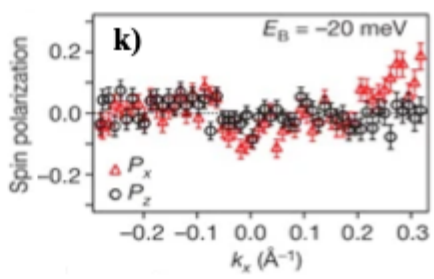

Figure 10: Photoemission data for $\mathrm{Bi}_{2} \mathrm{Se}_{3}$ and $\mathrm{Bi}_{2} \mathrm{Te}_{3}$. a) High-resolution ARPES of $\mathrm{Bi}_{2} \mathrm{Se}_{3}(111)$ (measured with incident photon energy of $22 \mathrm{eV}$ near the $\Gamma$-point along the $\Gamma-\mathrm{M} \Gamma$-K directions). b) DFT band structure: Bulk band projections represented by the shaded areas. The band-structure calculated with (without) SOC is presented in blue (green). Surface states with SOC (without SOC) are shown in red (black). c) ARPES measurements of band dispersions for $\mathrm{Bi}_{2} \mathrm{Te}_{3}$ along $\mathrm{K}-\Gamma-\mathrm{K}$ (top) and $\mathrm{M}-\Gamma-\mathrm{M}$ (bottom) directions. The bulk band dispersions are labeled as BCB and BVB, whereas the sharp V-shape dispersion comes from the surface state band (SSB). d) Photon energy-dependent FS maps. e) Tetradymitetype crystal structure of $\mathrm{Bi}_{2} \mathrm{X}_{3}(X$ $=\mathrm{Se}, \mathrm{Te}$ ), with typical layered structure and quintuple-layer stacking. Also shown is the bulk and surface Brillouin zone. f,g) ARPES intensity map at EF of the (111) surface of tuned stoichiometric $\mathrm{Bi}_{2-\delta} \mathrm{Ca}_{\delta} \mathrm{Se}_{3}$ and of $\mathrm{Bi}_{2} \mathrm{Te}_{3}$ (b). Red arrows denote the direction of spin projection around the Fermi surface. h,i) ARPES dispersion of $\mathrm{Bi}_{2-\delta} \mathrm{Ca}_{\delta} \mathrm{Se}_{3}$ and of $\mathrm{Bi}_{2} \mathrm{Te}_{3}$ (b) along the $k_{x}$ cut. The dotted red lines are guides to the eye. The shaded regions are projections of the bulk bands of pure $\mathrm{Bi}_{2} \mathrm{Se}_{3}$ and $\mathrm{Bi}_{2} \mathrm{Te}_{3}$, respectively, onto the (111) surface. j) Measured y component of spin-polarization along the $\Gamma-\mathrm{M}$ direction at an energy $-20 \mathrm{meV}$, which only cuts through the surface states. Inset, schematic of the cut direction. k) Measured $\mathrm{x}$ (red triangles) and z (black circles) components of spin-polarization. Panels a) and b) adapted with permission from Ref. ${ }^{49}$ Copyright 2009 Springer Nature; panels c), d) and e) adapted with permission from Ref., ${ }^{107}$ Copyright 2009 American Association for the Advancement of Science 3 AAAS); panels f)-k) adapted from Ref. ${ }^{106}$ Copyright 2009 Springer Nature 
Although the first explored example of TI was $\mathrm{Bi}_{x} \mathrm{Sb}_{1-x},{ }^{117,119}$ its complex surface structure and spectra, along with the small band gap, hindered the clear identification of topological phenomena at high temperatures. However, a search was launched towards TI with larger band-gaps and simpler surface electronic structures, which led to the identification of bismuth-based chalcogenides (layered-compounds with hexagonal symmetry and characteristic quintuple-layer structure) as TI prototypes. In that materials class, less-stringent conditions (i.e. observation of topologically non-trivial behaviour at room temperature and without magnetic fields for bare surfaces) really boosted the field of quantum topological matter. Indeed, the spin momentum locking, the non-trivial $\pi$ Berry phase and the robustness of TI features with respect to perturbation (i.e. non-magnetic disorder) were all demonstrated in landmark papers $\left({ }^{49,106,107}\right)$ on Bi-based chalcogenides, some of the results being summarized in Figure 10. In Figure 10 a) a clear Dirac cone along the $\Gamma-M$ and $\Gamma-K$ directions is evident for $\mathrm{Bi}_{2} \mathrm{Se}_{3},{ }^{49}$ which well compares with the corresponding band structure calculated by first-principles (Figure $10 \mathrm{~b}$ ). DFT well shows the effect of SOC, inducing both the band-inversion for the bulk case and the Dirac cone for the surface case (cfr Figure $10 \mathrm{c}$ ). This is an example of a weakly correlated material, so that the agreement between ARPES and DFT is indeed very good.

The case of $\mathrm{Bi}_{2} \mathrm{Te}_{3},{ }^{107}$ a well-studied material known for its thermoelectric properties, has allowed the exploration of chemical trends upon anion substitution (Se vs Te), leading to a smaller band gap, a significant deviation from a linear Dirac cone and a hexagonal warping potential, driving the change from a circular to star-shaped surface constant-energy maps (cfr Figure $10 \mathrm{c}$ )-d), where the apex of the V-shape dispersion shows the Dirac point). In Figure $10 \mathrm{~d}$ ), the shape of the inner Fermi surface (FS) strongly depends on photon energy, indicating a large out-of-plane dispersion and thus a strong bulk nature of these states, whereas the non-varying shape of the outer star-shaped FS with changing photon energy confirms its surface state origin. These findings also highlight the power of synchrotronbased photon-energy-dependent ARPES measurements, further strengthened - if available 
- by the combination of ARPES performed with vacuum ultraviolet and soft- X-ray light. The robustness of the Dirac cone upon chemical disorder is shown in Figure $10 \mathrm{f}$ ) and h), where spin-resolved ARPES based on Mott detectors is reported for Ca-doped $\mathrm{Bi}_{2} \mathrm{Se}_{3} .{ }^{106}$ In fact, often, $\mathrm{Bi}_{2} \mathrm{Se}_{3}$, as grown, is a doped semiconductor, with $n$-type character (i.e. chemical potential within the bulk conduction band) caused by Se vacancies. ${ }^{120}$ In order to move the Fermi level within the band gap, $\mathrm{Bi}_{2} \mathrm{Se}_{3}$ can be doped with small Ca concentrations, compensating for the anion vacancies. The measured spin-polarization along $x, y, z$ in $\mathrm{Bi}_{2} \mathrm{Te}_{3}$ (cfr Figure 10 j)-k)) show a clear polarization signal (i.e. equal magnitude but opposite signs) along the $y$-direction, whereas it vanishes along $x$ and $z$ directions. This is consistent with the spin-momentum locking: each momentum along the surface has a well-defined single spin state at the Fermi level, with the spin direction rotating as the momentum moves around the Fermi surface, leading to a non-trivial $\pi$ Berry phase. ${ }^{49,106,107}$

Due to limited space, only pioneering data were reported here for topological insulators. However, we conclude this section by noting that research on quantum topological matter is one of the most active fields in condensed matter physics nowadays, with many research lines being pursued (from topological magnets to Kondo TI, from topological crystalline insulators to topological superconductivity, etc). ${ }^{112,121-123}$

\subsubsection{Rashba materials}

Let us start this section by briefly recalling: $i$ ) spin splitting effects in materials' band structure, in terms of symmetry properties and $i i)$ what the Rashba effect (RE) ${ }^{124-126}$ is related to (see Figure 11). In the presence of Space Inversion Symmetry (SIS) and Time-Reversal Symmetry (TRS), each eigenstate, for every k-point in the Brillouin Zone, is doubly degenerate for spin (or, equivalently there are no spin splitting effects, cfr Figure 11 a). When TRS is kept but SIS is broken in a polar fashion (such as at surfaces, interfaces with twodimensional-electron-gas, bulk materials with polar axis, etc), there is a potential asymmetry (or, equivalently, an electric field $E_{0}$ ). In the relativistic approach and by means of Lorenz 
transformations, an electron travelling with a certain velocity (or momentum $\mathbf{k}$ ) will see the electric field as a magnetic field, which its spin will then couple to, via a Zeeman-like interaction. As such, the carrier will be subject to the so-called "Rashba interaction", ${ }^{124,125}$ producing - even in non-magnetic compounds - a spin-splitting of the eigenstates: at a certain k-point, the eigenvalues will be different for up-and down-spins. However, the splitting will be opposite at opposite momentum -k, so that no net magnetic moment arises in total (i.e. the Rashba is a $\mathbf{k}$-dependent spin-splitting). The Rashba interaction is usually modeled with a linear-term in momentum, thereby producing an opposite shift of the parabolic band structure for opposite spins (cfr Figure $11 \mathrm{~b}$ ), where the relevant parameters of the $\mathrm{RE}-E_{R}, k_{R}$ and $\alpha_{R}$ - are graphically explained). Neglecting terms at orders higher-thanlinear, in RE the spin is perpendicular to both electric field and momentum, leading to spin-momentum locking (similar to TI). In case a material, in addition to showing a polar axis, also shows switchable polarization (i.e. the material is ferroelectric), then an additional functionality emerges: ${ }^{127,128}$ the spin-polarization can be switched as the ferroelectric polarization is switched (cfr Figure $11 \mathrm{c}$ ), thereby leading to the class of FerroElectric Rashba Semiconductors (FERSC), that will be briefly discussed below.

Although the RE was heavily investigated for surfaces of heavy metals by means of ARPES, ${ }^{129-132}$ the situation shown in Figure 11 b) is by symmetry allowed in every system, provided a polar axis is present. Indeed, a breakthrough discovery for RE was achieved in BiTeI, ${ }^{133}$ the first bulk material where a sizeable RE was reported. BiTeI is characterized by a trigonal layered structure, with $\mathrm{Bi}, \mathrm{Te}$, and I planes stacked along the $z$ direction. In the pioneering work of Ishizaka et al. ${ }^{133}$ a very large Rashba effect was detected (cfr Figure 11 a)-c), from measurements of both band dispersions and constant energy maps, from ARPES and spin-resolved ARPES. In all cases, the picture was consistent with mostly "bulk"-derived Rashba states with $k_{R}=0.052 \AA^{-1}, E_{R}=0.1 \mathrm{eV}$ and $\alpha_{R}=3.8 \mathrm{eV} / \AA$. These parameters were among the largest ever measured for Rashba effects.

BiTeI was later reported to show an "ambipolar" behaviour in terms of carrier doping: ${ }^{134}$ 
a) No-spin splitting

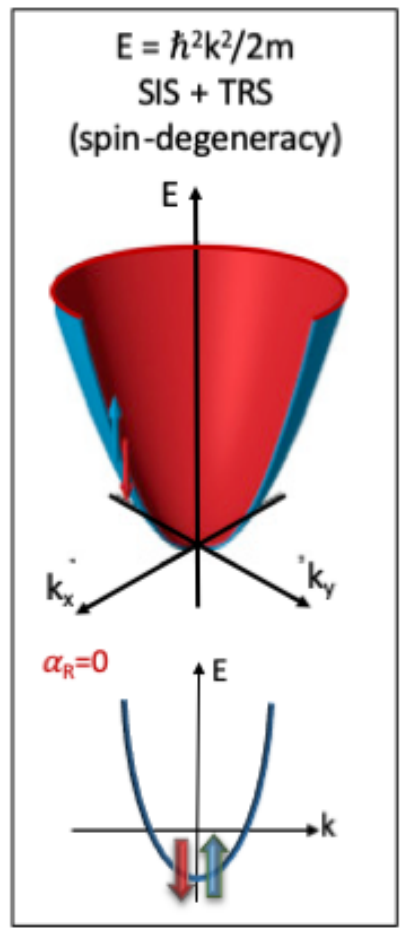

b) Rashba effect - Polar systems

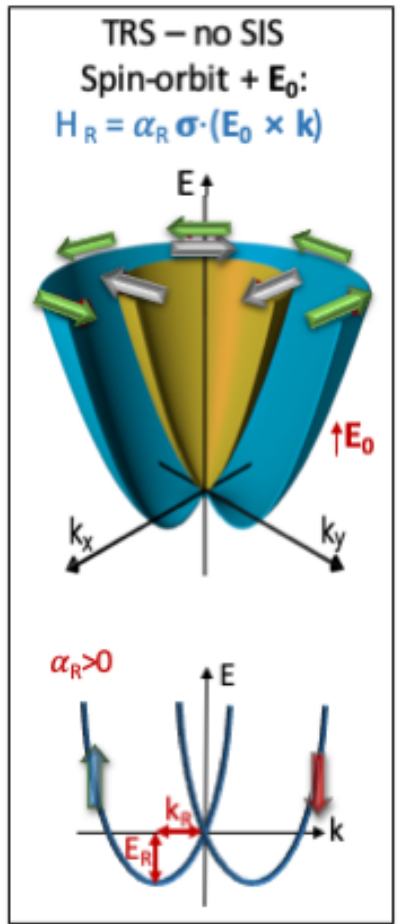

c) Rashba Effect in FERSC

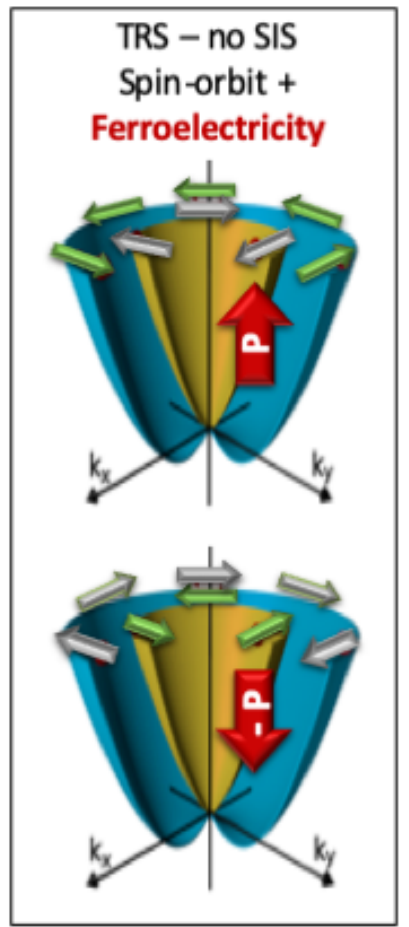

Figure 11: Rashba effect in polar systems and in Ferroelectrics. a) Spin degeneracy in systems where both TRS and SIS are kept (simple free-electron model for band-structure). Upper (lower) part shows a 3D view (2D cut). b) Polar systems, where SIS is lost but TRS is kept: the joint presence of SOC and electric field lead to the RE. Upper (lower) part shows a 3D (2D) view. The common parameters to quantify the $\mathrm{RE}$ are shown: Rashba momentum offset $k_{R}$ (i.e. difference in reciprocal space between the $\mathrm{k}$-point where the energy minimum occurs with respect to the high-symmetry k-point) and RE energy difference $E_{R}$ (i.e. difference in energies between the conduction band minimum and the energy where the two spin-split bands cross). The Rashba coefficient is defined as: $\alpha_{R}=2 E_{R} / k_{R}$. Arrows on the constant energy cuts represent the spin-texture [i.e. with clockwise (counterclockwise) circulation for the inner (outer) branch]. c) RE in FERSC (SIS broken, TRS kept): ferroelectric polarization (see vertical red arrow) switches the spin-texture (see opposite circulation for spin-textures in upper and lower parts). 

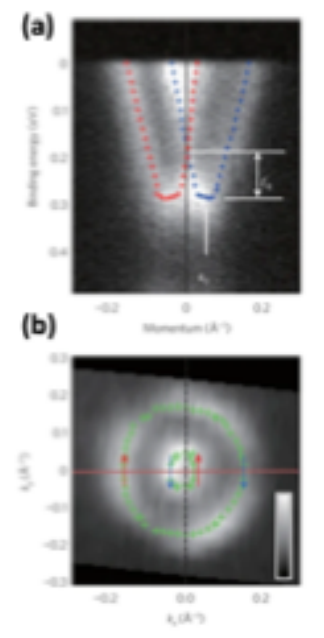
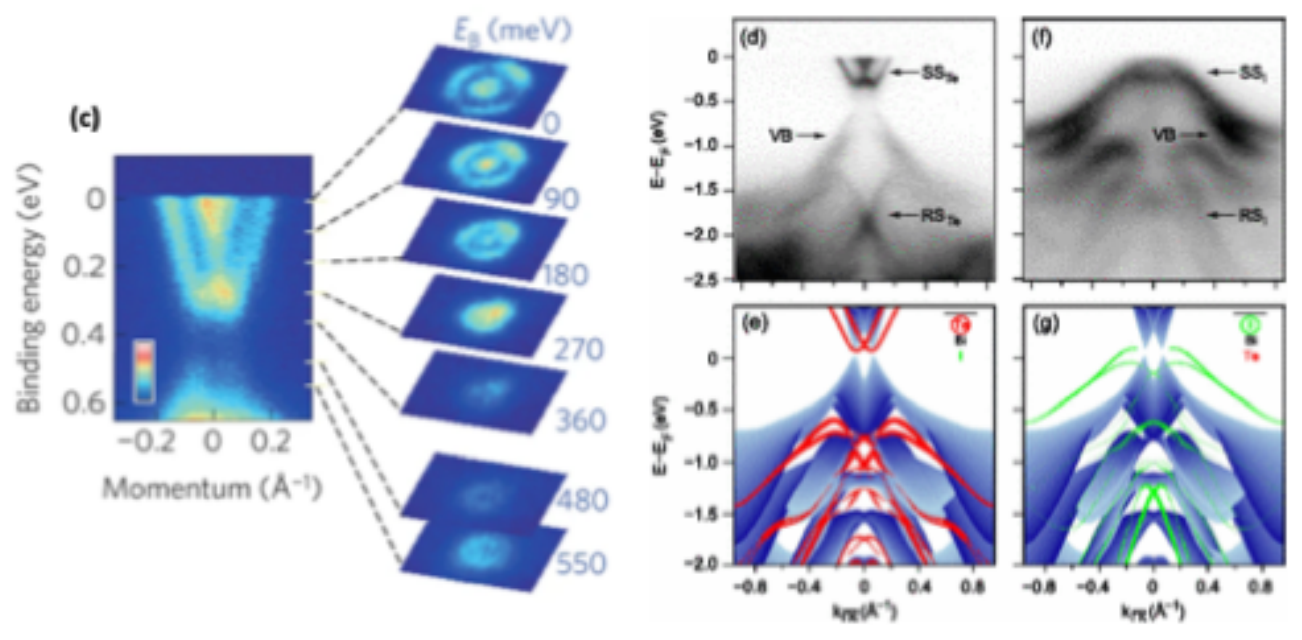

Figure 12: Rashba Effects in BiTeI. a) Spin polarization of Rashba-split bands. Markers tracking the band dispersions overlaid on the ARPES intensity image. Red (blue) markers represent the "spin-up" ("spin-down") components. b) Fermi-surface map. Green markers show the peak positions of momentum distribution curve at $E_{F}$. c) ARPES image of the Rashba-split conduction bands $(h \nu=21.2 \mathrm{eV})$. The right small panels show the band contour images as functions of $k_{x}$ and $k_{y}$ at certain binding energies. d) ARPES dispersion for a Te-terminated surface along $\Gamma-\mathrm{K}$ measured at $93 \mathrm{eV}$ and $\mathrm{T}=40 \mathrm{~K}$, compared to e) projected-slab DFT band structure. f) and g) show corresponding plots for the I-terminated surface. The size of the red (green) symbols in e) [in g)] is proportional to the contribution of the surface Te (I) atoms. The continuum of bulk states is shown in blue. In (e) and (g) only projection amplitudes larger than 0.1 are shown. Panels a),b) and c) adapted with permission from Ref., ${ }^{133}$ Copyright 2011 Springer Nature; panels d)-h) reproduced with permission from Ref., ${ }^{134}$ Copyright 2012 American Physical Society.

upon control of the ionic surface termination (Te vs I), the chemical potential could be placed in either the conduction or the valence band. In both cases, a giant spin splitting at the Fermi level was observed, arising mostly from surface states, though even bulk states contributed to the signal. Indeed, in Figure 12 d) a split parabolic band crossing the Fermi level is evident. The measured Rashba parameters were reported to be $E_{R}=0.32 \mathrm{eV}$ and $k_{R}=0.055 \AA^{-1}$, the RE occurring close to the BZ center $\Gamma$. The measured RE for BiTeI was therefore one magnitude larger than for the prototypical metal surfaces ${ }^{129-132}$ and for systems showing a two-dimensional electron-gas. ${ }^{135}$

The comparison between ARPES and the first-principles band structure [cfr Figure 12 e)] for BiTeI slabs was found to be very good. ${ }^{134}$ The calculations showed, in particular, that both surface and the bulk states are strongly split by SOC and that the most evident spin-split state is localized in the topmost bilayer, with partial overlap to conduction band states showing a smaller $k_{R}$. When moving to the I-terminated surface, ${ }^{134}$ the picture was 
reported to be qualitatively and quantitatively different: electron pockets were replaced by hole pockets from a spin-split state (labeled as SSI in Figure $11 \mathrm{f}$ ) with a strong weight on the surface I atoms and a large momentum offset $\left(k_{R} \sim 0.2 \AA^{-1}\right)$. A precise determination was found to be difficult, due to the top of the band located in energy above $E_{F}$, highlighting the $p$-type character of the I-terminated surface and therefore confirming the ambipolar behaviour in BiTeI. ${ }^{134}$ Subsequent studies also showed how the spin-splitting of the electronic states in BiTeI is accompanied by the development of momentum-dependent orbital textures, demonstrated via variable light polarisation measurements, and again in good agreement with the conclusions of density-functional calculations. ${ }^{136}$

After BiTeI, another important step was taken in 2013, when GeTe was put forward ${ }^{127,128}$ as an important bulk material with strong RE. GeTe is a ferroelectric semiconductor showing extremely large Curie temperature $\left(T_{C}=720 \mathrm{~K}\right)$, space group $R 3 m$, i.e. rhombohedrallydistorted rocksalt structure and ferroelectric polarization along the [111] axis. ${ }^{139}$ Ab-initio simulations ${ }^{127}$ predicted not only GeTe to show a huge RE (with $k_{R}=0.09 \AA^{-1}, E_{R} \sim 0,2$ $\mathrm{eV}$ and $\alpha_{R}=4.8 \mathrm{eV} / \AA$, parameters even larger than BiTeI), but also that the spin-texture could be reversed upon polarization switching (i.e. applying an opposite electric field). GeTe was therefore considered as a prototypical material of the FERSC class. Soon after the DFT predictions, an experimental confirmation was published: ${ }^{138}$ epitaxial (111) GeTe thin films were deposited on $\mathrm{Si}(111)$ by means of Molecular Beam Epitaxy ${ }^{140}$ and confirmed to be ferroelectric via piezo-force microscopy ${ }^{138}$ with ferroelectric polarization pointing outwards the surface. ARPES measurements (with and without spin-resolution) ${ }^{138,141}$ clearly showed Rashba-like valence bands (cfr Figure 13). The spectra show a marked $p$-type behaviour, with large concentration of holes arising from easily-created Ge vacancies. The theoryexperiments comparison allowed to disentangle surface states and bulk bands [cfr Figure 13 e)-f)-g)-h)] and to conclude that GeTe shows a giant RE both for surface and bulk bands. Finally, the spin texture measurements ${ }^{138}$ revealed spins directed predominantly perpendicular to the corresponding momentum, consistent with DFT predictions for an outward 
(a)
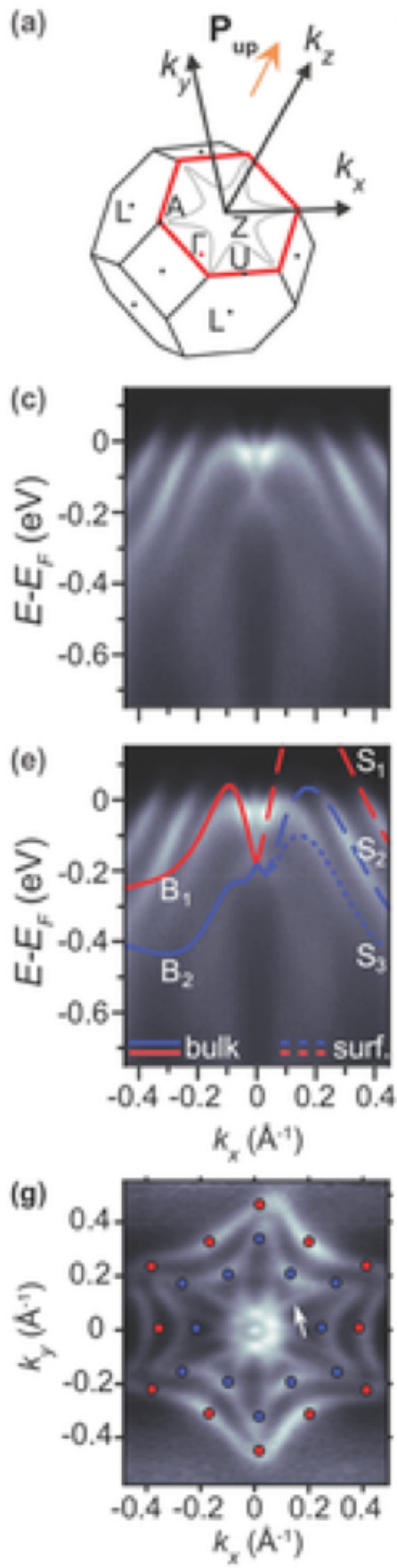

(b)
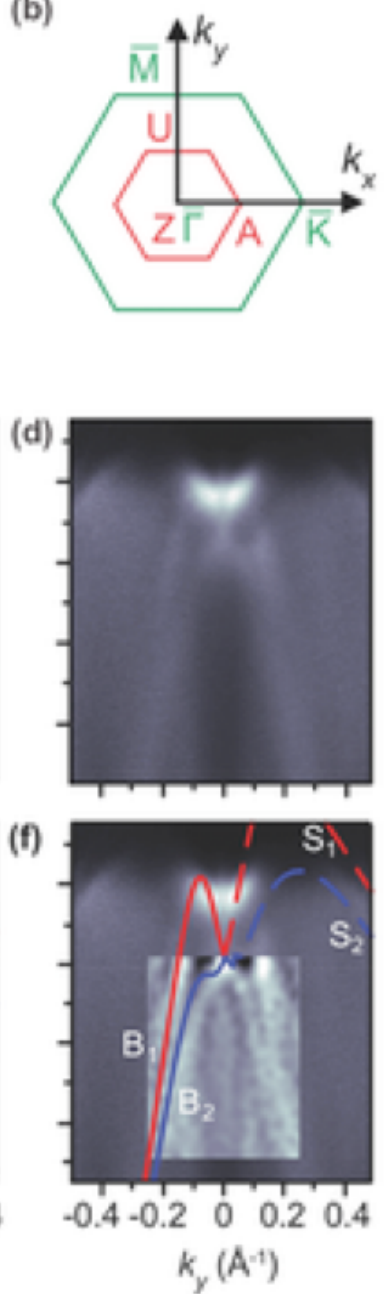

(h)

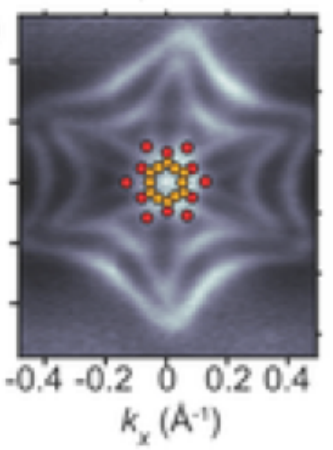

Figure 13: Rashba effects in GeTe. a) GeTe Brillouin zone. The direction corresponding to polarization, the related perpendicular plane and the relevant A-Z-U directions are highlighted. b) Surface Brillouin zone (green hexagon) and projection of the hexagonal face of the bulk Brillouin zone (red hexagon). c) ARPES data along $\Gamma-\mathrm{K}(\mathrm{ZA})$ at photon energy $h \nu=20 \mathrm{eV}$. d) Same as (c) along $\Gamma-\mathrm{M}$ (ZU). e) Same as (c) with overlaid DFT-calculated bulk bands (full lines labeled $\mathrm{B}_{i}$, left side of the panel) and surface bands (dashed/dotted lines labeled $S_{i}$, right side of the panel) from the relaxed slab calculation. f) Same as (e) along $\Gamma-\mathrm{M}(\mathrm{ZU})$, partly using two-dimensions curvature representation ${ }^{137}$ (see inset). g,h) Photoelectron intensity distribution in the $\left(k_{x}, k_{y}\right)$ plane at $E_{F}$ with overlaid DFT-calculated (g) surface states and (h) bulk states (dots) along the high symmetry directions. Reproduced with permission from Ref., ${ }^{138}$ Copyright 2016 Wiley-VCH. 
a Transition metal Chalcogen $2 \mathrm{H}$

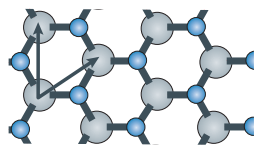

${ }^{A}$ की
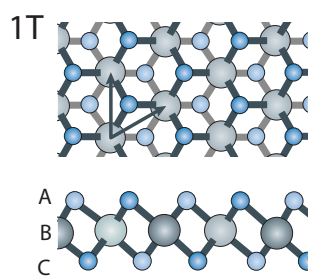

$1 \mathrm{~T}^{\prime}$

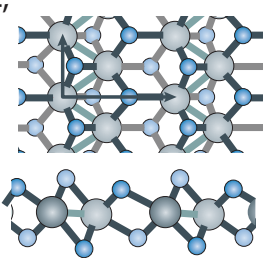

b
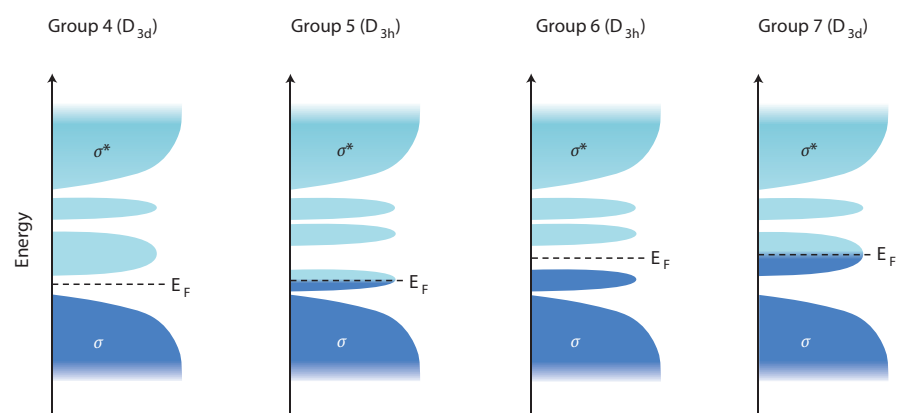

C

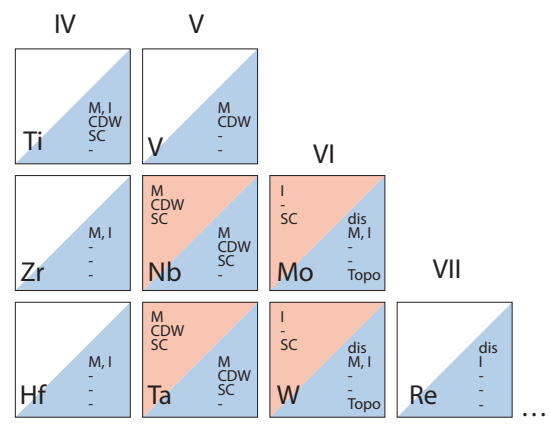

\begin{tabular}{ll}
\hline 2H & $\square 1 T$ \\
dis & Distorted \\
M & Metallic \\
I & Insulating \\
CDW & Charge density wave \\
SC & Superconducting \\
Topo & Topological
\end{tabular}

Figure 14: Transition-metal dichalcogenides. (a) Top and side view crystal structures of the $2 \mathrm{H}, 1 \mathrm{~T}$, and 1T' polymorphs of TMDs. Only one of two layers within the bulk unit cell is shown for the $2 \mathrm{H}$ structure. (b) Schematic evolution of electronic structure across the series, dominated by the varying occupation of d-states located between the bonding $(\sigma)$ and antibonding $\left(\sigma^{\prime}\right)$ chalcogen-derived bands. $\mathrm{D}_{3 \mathrm{~d}}$ and $\mathrm{D}_{3 \mathrm{~h}}$ refer to the point group of the transitionmetal site in a trigonal prismatic or trigonal antiprismatic (approximating to octahedral) coordination within a monolayer geometry. (c) Wide variety of materials properties that are realised for different transition-metal choices across the $\mathrm{MX}_{2}$ series. Panels (a,c) adapted with permission from Ref., ${ }^{142}$ copyright 2017 Springer Nature; panel(b) adapted with permission from Ref., ${ }^{143}$ Copyright 2013 Springer Nature.

polarization and reinforcing the overall picture of a huge RE.

\subsection{Transition-metal dichalcogenides}

The transition-metal dichalcogenides (TMDs, Figure 14) host a rich and diverse structural chemistry based on a large number of polytypes, and a wide range of electronic behaviours. ${ }^{142}$ In the $2 \mathrm{H}$ structure adopted by $d^{1}$ and $d^{2}$ dichalcogenides of group 5 and 6 and typified by $M o S_{2}$, the chalcogen X atoms form hexagonal layers stacked in a sequence AA BB AA 
etc. The metal atoms M (conventionally represented by lower case letters in the chemical literature, although this convention is not used in many recent publications) occupy sites with trigonal $D_{3 h}$ symmetry between the AA or BB layers and the unit cell contains two $\mathrm{MX}_{2}$ units, the overall ordering running $\mathrm{AbA} \mathrm{BaB}$ in $2 H_{1}$ and $\mathrm{AcA} \mathrm{BcB}$ in $2 H_{2}$. The structural variant $3 \mathrm{R}$ has an anion sequence $\mathrm{AA}$ BB CC and a cation sequence $b c a$. The alternative single layer $1 \mathrm{~T}$ structure (usually referred to as the $C d I_{2}$ structure in the chemical literature) again involves hexagonal chalcogen layers, but now stacked in a sequence AB AB. This gives overall hexagonal close packing of the anions in three dimensions. The cations occupy trigonal antiprismatic c sites of $D_{3 d}$ symmetry between the AB planes, approximating to $\mathrm{O}_{\mathrm{h}}$ locally and so the overall stacking sequence is AcB AcB. The cations therefore form 1D columns, thus allowing the possibility of metal-metal bonding as in the distorted $1 T^{\prime}$ structure. The M-X bonds are predominantly covalent, while those between neighbouring $\mathrm{MX}_{2}$ layers are predominantly van der Waals in character.

As shown schematically in Figure 14, the essentially octahedral ligand field in the $1 \mathrm{~T}$ structure splits the $d$ levels into a lower 3 -fold degenerate $t_{2 g}$ band derived from $d_{x y}, d_{x z}$, and $d_{y z}$ orbitals and a 2-fold degenerate $e_{g}$ band composed of $d_{z^{2}}$ and $d_{x^{2}-y^{2}}$ orbitals: lowering the symmetry to $D_{3 d}$ further splits $t_{2 g}$ into $a_{1 g}+e_{g}$ (although this splitting is expected to be very small and is ignored in the schematic energy level diagram of Figure 14), while the upper $e_{g}$ manifold remains unsplit. In a $D_{3 h}$ coordination environment a different 1:2:2 splitting pattern is found with an orbital sequence $a_{1}^{\prime}\left(d_{z^{2}}\right), e^{\prime}\left(d_{x y}, d_{x^{2}-y^{2}}\right)$, and $e^{\prime \prime}\left(d_{x z}, d_{y z}\right)$. Note that Figure 14 assumes that the metal d bands all lie above bonding chalcogen-derived $p$ bands. As will be discussed below, this is not always the case and the possibility of band overlap adds an extra level of complexity to unravelling the electronic structure of these materials.

The chalcogen site is normally occupied by one of S, Se, or Te, although alloys and in some cases ordered layer-by-layer combinations of different chalcogens can be created. ${ }^{144}$ The transition-metal site can be filled by a wide array of different elements, leading to a 
rich and diverse materials chemistry in this system. Broadly classified by their d-electron occupation (Figure 14b), ${ }^{143}$ these systems range from metals to semiconductors, and are found to exhibit a host of exotic physical properties including superconductivity, chargedensity wave order, and non-trivial band topology (Figure 14c). ${ }^{142,143,145-155}$ The layered nature of the crystal structure allows them to be cleaved easily, enabling preparation of flat and clean surfaces. As such, the TMDs have provided ideal systems for study using angleresolved photoemission, which has in turn played a crucial role in elucidating the electronic structure and interacting states in such systems. Moreover, being easy to exfoliate even down to the single-layer limit, TMDs form the parent compounds for a wide range of 2D materials beyond graphene. ${ }^{142,143,145}$ They have thus become prototypical systems in which to test the rapidly-developing capabilities of spatially-resolved, so-called "nano-ARPES" experiments. In this section, we will discuss some of the insights that can be gained into this rich materials system by ARPES studies, including those which exploit spin-, spatial-, or temporal- resolution.

\subsubsection{Chemical trends}

As introduced above, a significant number of the TMDs are semiconducting (Figure 14). ARPES provides direct access only to the occupied electronic states, and as such can be used to directly probe the momentum-space location and structure of the uppermost valence bands in the system. Moreover, via some form of chemical doping, it can yield similar information regarding the bottom of the conduction band, placing constraints on band gap sizes and, importantly, resolving the direct or indirect nature of such semiconducting band gaps, and chemical trends of these.

As an example, we show in Figure 15, ARPES measurements of the group-IV chalcogenides $\mathrm{TiS}_{2}, \mathrm{TiSe}_{2}$, and $\mathrm{TiTe}_{2}$. Within an ionic picture, the chalcogen would be expected in a $2^{-}$charge state, rendering the Ti in a $4^{+}$state and a nominal $3 d^{0}$ configuration. This simple picture would suggest that these systems are semiconductors, with a valence band derived 

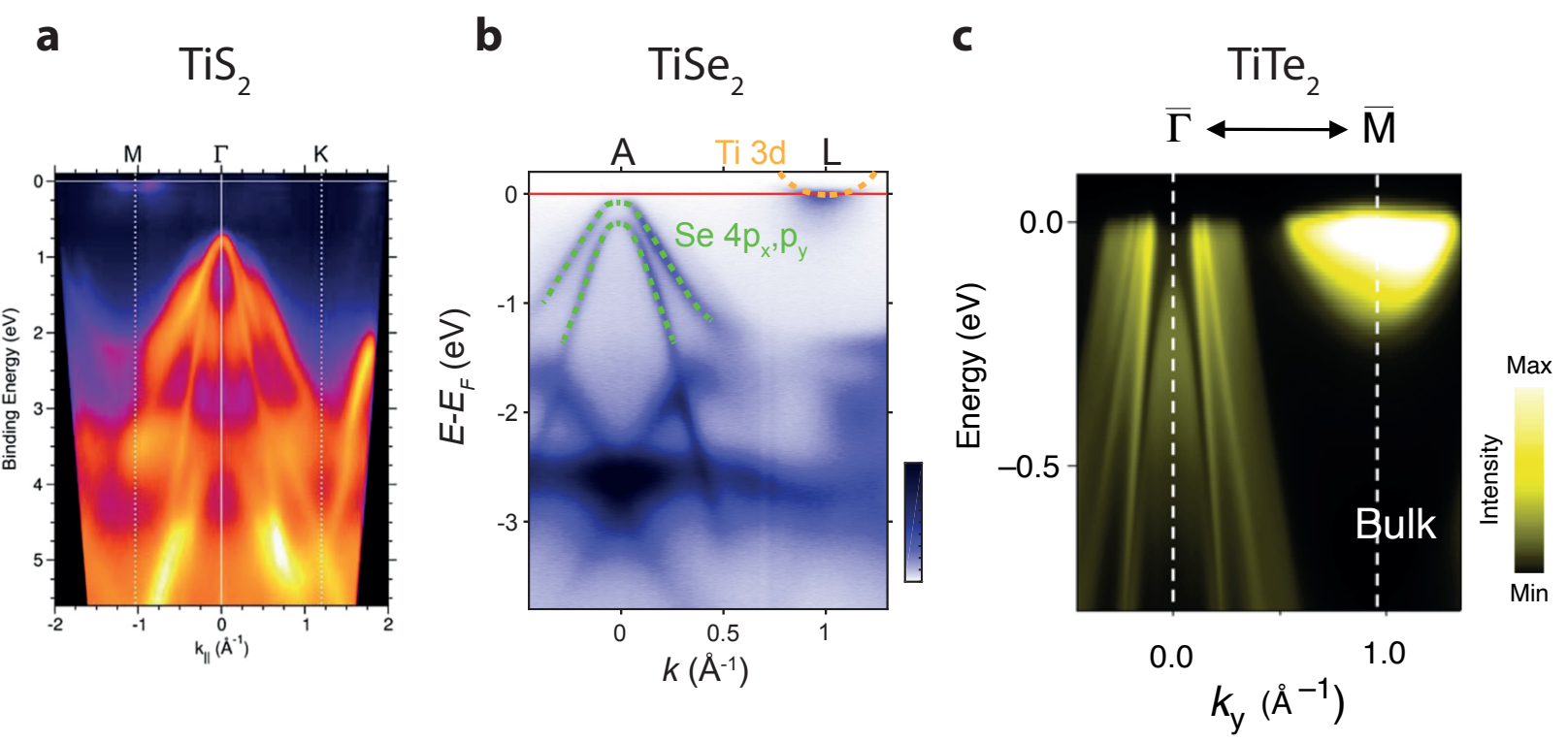

Figure 15: Band structure evolution across the $\mathrm{TiX}_{2}$ series. ARPES measurements showing the electronic structure of (a) $\mathrm{TiS}_{2}$, (b) $\mathrm{TiSe}_{2}$ and (c) $\mathrm{TiTe}_{2}$. Reproduced with permission: panel (a) from Ref. ${ }^{156}$ and panel (c) from Ref., ${ }^{157}$ both under Creative Commons International License, panel (b) from Ref. ${ }^{158}$ )

from chalcogenide $p$-orbital states and an empty Ti $3 d$-derived conduction band. Transport measurements, however, typically indicate temperature-dependent resistivities indicative of a metallic state across the family of $\mathrm{TiX}_{2}$ systems, ${ }^{159-162}$ which called into question the nature of the ground state in these systems.

Figure 15a shows how in $\mathrm{TiS}_{2}$ the metallic transport derives from unintentional electron doping in the system. ${ }^{156}$ ARPES measurements show a highly dispersive set of states at the Brillouin zone centre, which can be attributed to the p-orbital states of S. The band top of these states is almost $1 \mathrm{eV}$ below the Fermi level. At the M-point (centre of the Brillouin zone face), a barely-occupied band is visible at the Fermi level. This has a much flatter dispersion, indicating a heavier effective mass, and can be assigned as the bottom of the Ti d-derived conduction band. While this is nominally unoccupied, unintentional doping leads to a partial occupation of these states and hence metallic transport. Fundamentally, however, the ARPES measurements indicate that this system should be considered as an indirect band-gap semiconductor, with conduction and valence band extrema located at 
different k-space locations. In $\mathrm{TiTe}_{2}$, on the other hand, the Te-derived valence band width can be expected to be larger due to the larger Te $5 p$-orbitals as compared to the $\mathrm{S} 3 p$-orbitals in $\mathrm{TiS}_{2}$, while the lower electronegativity of Te will promote $d-p$ charge transfer. ${ }^{163}$ ARPES measurements of $\mathrm{TiTe}_{2}$ (e.g. Figure 15c, Ref. ${ }^{157}$ ) indicate that this causes the top of the Te $5 p$-orbital derived manifold to be moved above the bottom of the Ti $3 d$-derived states. The top of the valence band thus moves above the Fermi level, while the conduction band minimum is now intrinsically located below the Fermi level, and the system is thus best described as a semimetal, where the metallic transport is now truly intrinsic.

The Se-based member of the family is an interesting case. From the chemical trends evident from the S- and Te-based systems, it is not clear if the system would be nominally semiconducting as in $\mathrm{TiS}_{2}$, or semimetallic as in $\mathrm{TiTe}_{2}$. The presence of unintentional doped carriers and the indirect nature of the band gap in $\mathrm{TiS}_{2}$ precludes both transport and optical spectroscopy from providing an unambiguous answer to this question. In fact, ARPES measurements indicate that $\mathrm{TiSe}_{2}$ lies right on the borderline between the two cases, with previous ARPES measurements concluding results ranging from an indirect band gap of $150 \mathrm{meV}$ to a band overlap of $70 \mathrm{meV} .{ }^{158,164-168}$ Recent measurements using photon energies chosen to probe the true high-symmetry points of the three-dimensional Brillouin zone (see Figure 15b) show that there is in fact a small but non-vanishing indirect band gap of $74 \pm 8 \mathrm{meV}$ at room temperature, ${ }^{158}$ and indeed the proximity of $\mathrm{TiSe}_{2}$ to a zero-gap semiconductor has caused significant interest in $\mathrm{TiSe}_{2}$ as a possible host of a so-called excitonic insulator phase. ${ }^{169}$ As for $\mathrm{TiS}_{2}$, unintentional doping leads to a finite occupation of the conduction band, and hence metallic transport at low temperature, while temperaturedependent chemical potential variations, leading to changes in electron and hole populations, complicate the interpretation of transport at elevated temperatures. ${ }^{162}$

Rather than metallicity arising from unintentional doping, the group-V TMDs would be expected to be robust electrical conductors, with the transition metal in a nominal $d^{1}$ metallic configuration (Figure 14(b)). Resistivity measurements of $2 \mathrm{H}-\mathrm{NbSe}_{2}$ and $2 \mathrm{H}-\mathrm{TaSe}_{2}$ indeed 
a

$$
2 \mathrm{H}-\mathrm{TaSe}_{2}
$$

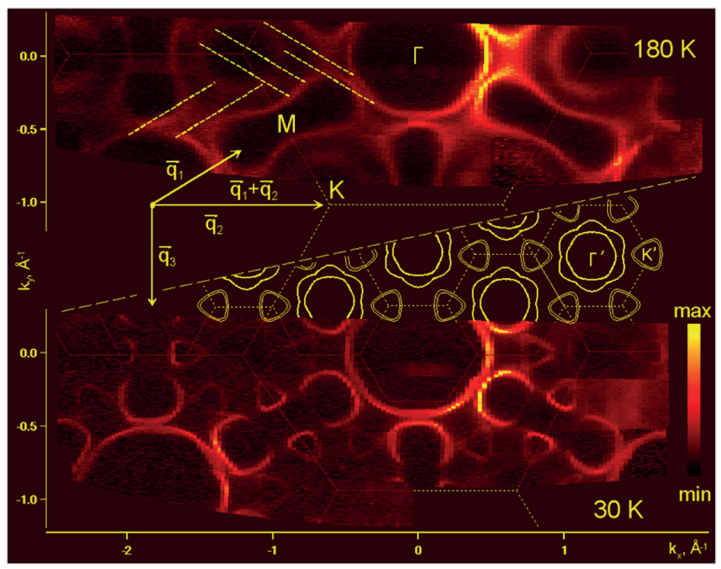

b

\section{$2 \mathrm{H}-\mathrm{NbSe}_{2}$}

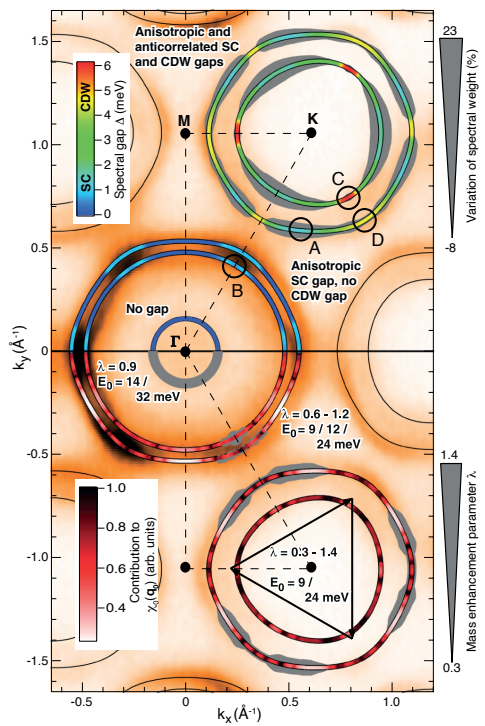

Figure 16: (a) Fermi surface of $2 \mathrm{H}-\mathrm{TaSe}_{2}$ above (top) and below (bottom) the CDW transition, showing the induced Fermi surface reconstruction. Reproduced with permission from ref., ${ }^{148}$ Copyright 2008 American Physical Society. (b) Fermi surface of $2 \mathrm{H}-\mathrm{NbSe}_{2}$ measured using ARPES. The underlying momentum-dependent spectral gaps $(\Delta)$, superconducting (SC) coherent spectral weight, mass enhancement parameter from electron-phonon coupling $(\lambda)$ and the contributions to the bare susceptibility $\left[\chi_{0}\left(\mathbf{q}_{0}\right)\right]$ are indicated, as extracted from detailed analysis of temperature-dependent ARPES data discussed in Ref. ${ }^{170}$ Reproduced with permission from Ref. ${ }^{170}$ Copyright 2012 American Physical Society.

show metallic temperature-dependence. ${ }^{171}$ With cooling, both systems transition first into a charge density-wave state and then become superconducting below a transition temperature of $7.3 \mathrm{~K}$ and $\approx 0.2 \mathrm{~K}$, respectively. As such, they have generated intense interest as systems in which to probe the co-existence or competition of superconductivity and charge-order. ARPES has played a crucial role in such studies. Figure 16, for example, shows the evolution of the Fermi surface of $2 \mathrm{H}-\mathrm{TaSe}_{2}$ upon cooling through the CDW transition temperature. $2 \mathrm{H}-\mathrm{TaSe}_{2}$ exhibits an incommensurate $\mathrm{CDW}$ at a temperature of $122 \mathrm{~K}$, followed by a lock-in transition to a commensurate $3 \times 3 \mathrm{CDW}$ at $90 \mathrm{~K} .{ }^{171}$ The multi-band nature of the normalstate Fermi surface is immediately clear. Its momentum-space structure does not show large parallel sections of the form that are often assumed to trigger a CDW transition via a 
Peierls-like mechanism. Yet, a clear and strong Fermi surface reconstruction is evident below $T_{\mathrm{CDW}}$, with a momentum-space periodicity reflecting the $3 \times 3$ real-space ordering. There has been extensive investigation into whether nesting hot spots can explain the origin of the transition, with recent studies favoring a momentum-dependent electron-phonon coupling as the key driver of the phase transition. ${ }^{172-175}$

The superconducting transition temperature of $2 \mathrm{H}-\mathrm{TaSe}_{2}$ is too low to be accessible in an ARPES experiment. In $2 \mathrm{H}-\mathrm{NbSe}_{2}$, on the other hand, the superconducting transition temperature is higher, but the CDW temperature is lower. The energy-scale of the corresponding CDW gaps opened at the Fermi level is thus smaller than in $\mathrm{TaSe}_{2}$. In a tour de force experiment performed at $1 \mathrm{~K}$ (which is at the lowest currently-available temperature for performing ARPES), Rahn et al. ${ }^{170}$ were able to resolve the full momentum-space gap structure of both the superconducting and CDW gaps and the $\mathbf{k}$-dependent electron-phonon coupling strength. A summary of their results, extracted from extensive temperature and momentum-dependent ARPES investigations, is shown in Figure 16b. Of particular importance, these highlight a key role of momentum-space anisotropy: the CDW and superconductivity both lead to a gapping of the Fermi surface. It is evident from the upper panel in Fig. 16b, however, that the CDW gap (the gaps that open of larger magnitude) is localised in isolated momentum-space regions. This thus leaves most of the Fermi surface 'available' for superconductivity, helping to explain the relatively high superconducting $T_{\mathrm{c}}$ in this system.

ARPES has also played a valuable role in helping to explain where systems deviate from the expected chemical trends shown in Figure 14(b). For example, from the simple crystal field arguments introduced above, the group-VII TMDs would be expected to be metallic. In contrast, systems such as $\mathrm{ReS}_{2}$ and $\mathrm{ReSe}_{2}$ are known to be semiconductors. For these material, the formation of Re-Re metal-metal bonds as discussed earlier leads to a distortion of the $1 \mathrm{~T}$ structure ${ }^{178,179}$ and formation of zigzag Re chains. ARPES measurements shown in Figure 17a indicate the influence of this pronounced structural distortion on the electronic structure. ${ }^{176,180,181}$ As well as becoming gapped, and thus semiconducting, the electronic 


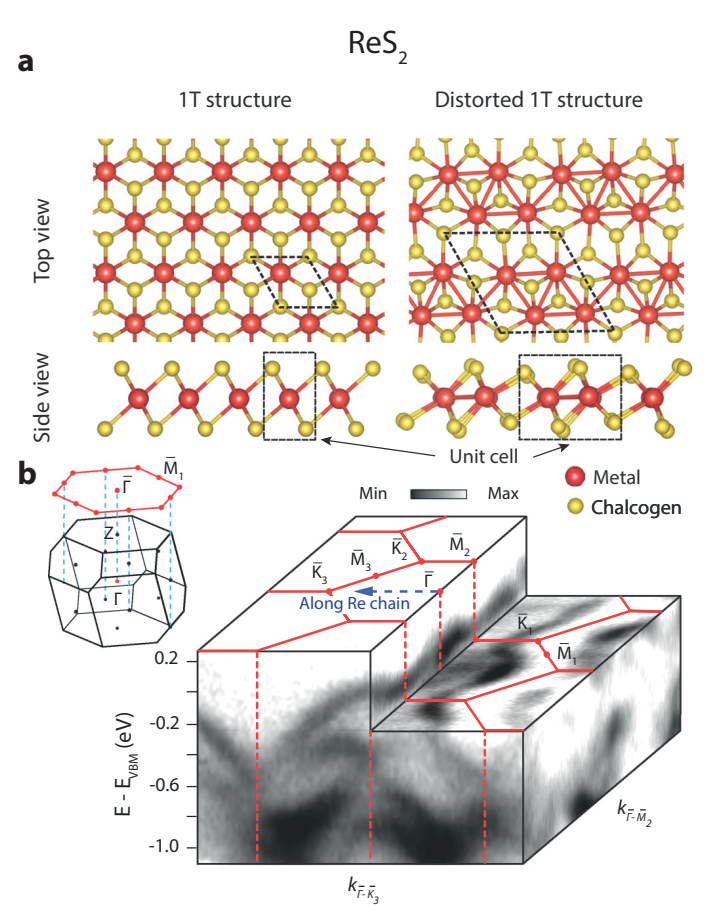

c $\quad \mathrm{PtSe}_{2}$

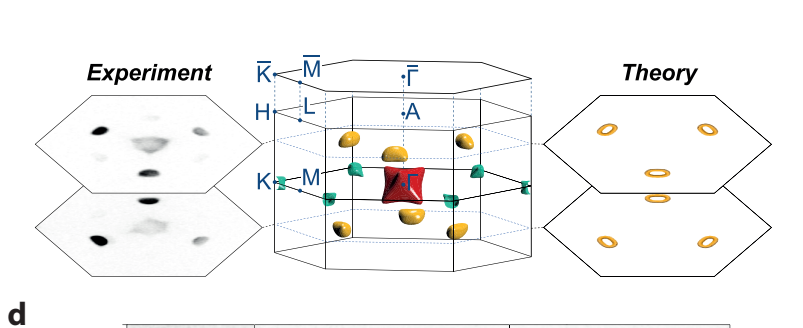

d

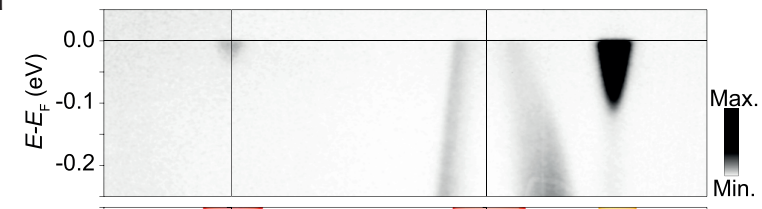

Figure 17: (a) Hypothetical pristine 1T crystal structure and actual distorted 1T crystal structure of $\mathrm{ReS}_{2}$. (b) Corresponding valence band electronic structure, showing quasi-1D band dispersions indicative of the chain-like structure in the distorted phase. Reproduced with permission from Ref. ${ }^{176}$ Copyright 2017 American Physical Society. (c) Experimental and theoretical Fermi surface of $\mathrm{PtSe}_{2}$ and (d) corresponding near- $E_{F}$ band structure. Reproduced with permission from Ref. ${ }^{177}$ Copyright 2019 American Physical Society.

structure now becomes strongly 2-fold symmetric. Quasi-1D dispersions reflect a much higher probability of electron hopping along the Re-Re chain direction than between the chains. This in turn, helps to explain the extremely anisotropic optical and electronic transport properties of these systems. ${ }^{182,183}$

In the opposite sense, in the simplest picture, $1 \mathrm{~T}$ group $\mathrm{X}$ systems such as $\mathrm{PtSe}_{2}$ and $\mathrm{PdTe}_{2}$ should be non-metallic with a completely filled $t_{2 g}$ band arising from the $5 d^{6}$ configuration. In contrast, as seen for $\mathrm{PtSe}_{2}$ in Figure $17 \mathrm{c}, \mathrm{d}$, dispersive states cross the Fermi level. ${ }^{177}$ From comparison with DFT calculations, which show excellent agreement with the measured electronic structure, these can be assigned as having dominantly Se $4 p$ oribtal character. There is strong hybridisation, but the transition-metal states do not play the major role at the Fermi level; rather the nominally bonding and anti-bonding branches of the 
chalcogen $p$-states overlap, leading to a semi-metal state. While typically it is the transitionmetal $d$ states of the TMDs which receive the most attention, the $p$-states can also thus play an important role in the materials physics and chemistry of the TMDs, and we will return to this point when discussing topological phases below.

\subsubsection{Dimensional-crossovers}

For the group-VI TMDs in the $2 \mathrm{H}$ polytype, the Fermi level sits in a gap of the crystal field-split Mo/W $d$-electron states, between $a_{1}^{\prime}$ and $e^{\prime}$ bands. As expected, these systems are therefore semiconducting (Figure 14). However, they exhibit a particularly interesting evolution of the band gap as a function of changing material thickness. In the bulk, they are known to exhibit only weak photoluminescence, as a result of the indirect nature of their fundamental band gap. A marked increase in luminescence efficiency is, however, observed when they are thinned to a single layer (one $\mathrm{MX}_{2}$ unit) in thickness, which has been attributed as resulting from a crossover to a band gap which is direct in momentum space. ${ }^{149,184}$

ARPES can again give fundamental insights into the origins of this behaviour. Figure 18a shows the measured electronic structure of bulk $\mathrm{WSe}_{2}$ along the $\bar{\Gamma}-\overline{\mathrm{K}}$ direction of the surfaceprojected Brillouin zone (Brillouin zone centre to corner). ${ }^{60}$ The uppermost valence bands have a multi-valley structure, with local maxima present at different locations in momentum

space. At the Brillouin zone corners ( $\overline{\mathrm{K}}$ and its time-reversed partner, $\left.\overline{\mathrm{K}^{\prime}}\right)$, a pair of sharp bands are visible, vertically shifted in energy by around $500 \mathrm{meV}$. Approaching the Brillouin zone centre, these bands disperse towards each other, before evolving into another band maximum at the $\bar{\Gamma}$ point, albeit with a spectral weight distribution in the ARPES which is significantly broader than at the Brillouin zone corners. This reflects an intrinsic difference in the dimensionality of the uppermost valence states at different locations throughout the Brillouin zone, as evident in the DFT calculations also shown in Figure 18a. The states at the zone corners show essentially no dispersion with changing value of the out-of-plane 

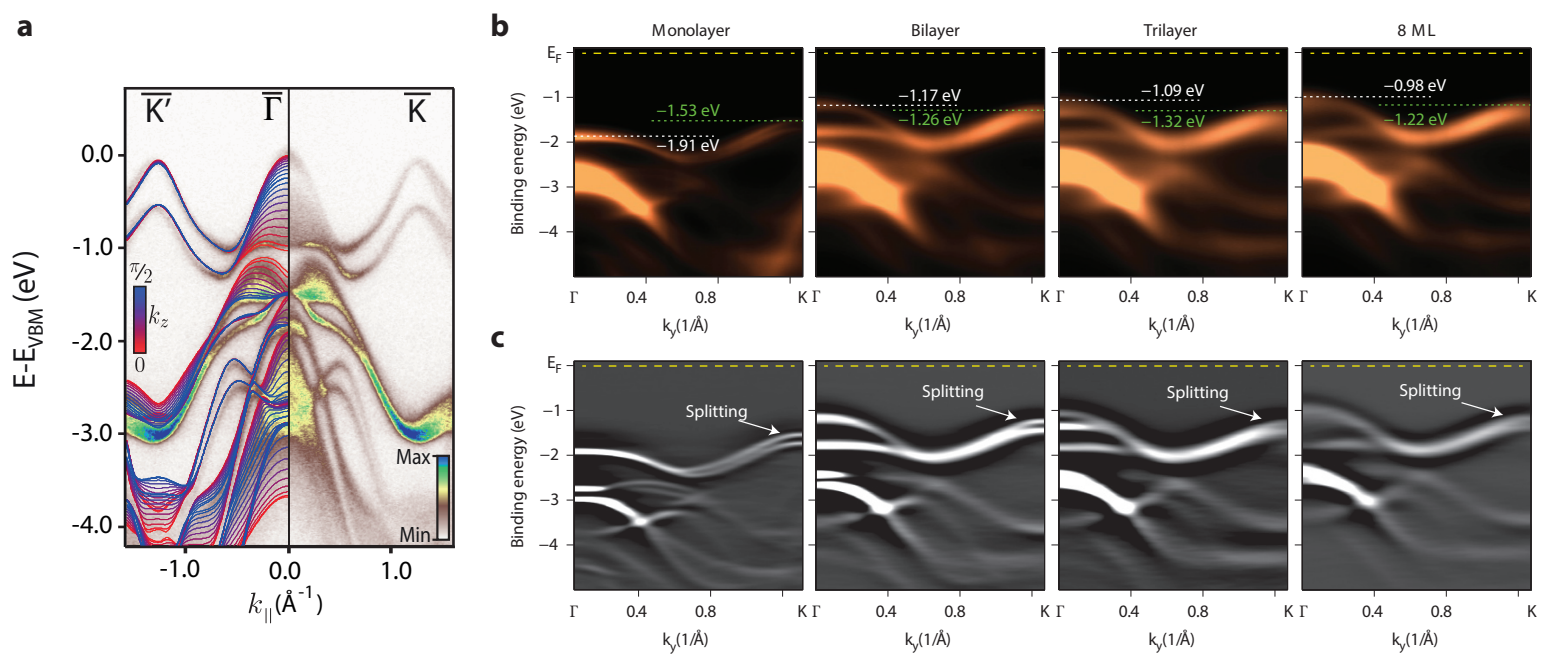

Figure 18: Thickness-dependent electronic structure of $\mathrm{WSe}_{2}$ and $\mathrm{MoSe}_{2}$. (a) ARPES measurements of the bulk band dispersions of $\mathrm{WSe}_{2}$. The results of DFT calculations are also shown on the left hand side, with each coloured line reflecting the calculated band dispersion for a different value of the out-of-plane momentum. Adapted with permission from Ref. ${ }^{60}$ Copyright 2014 Springer Nature. (b) ARPES measurements of thin-film $\mathrm{MoSe}_{2}$ grown by molecular-beam epitaxy, as a function of film thickness from 8 layer to monolayer (one ML $=$ one $\mathrm{MoSe}_{2}$ layer). (c) Corresponding second-derivative analysis of the data shown in (b), better highlighting the band dispersions. (b,c) Adapted with permission from Ref. ${ }^{185}$ Copyright 2014 Springer Nature.

momentum (different coloured lines), and so can be considered as two-dimensional. The states nearer to the zone centre, on the other hand, exhibit large changes in their corresponding calculated band dispersion with changing out-of-plane momentum. This traces back to a variation in orbital content of the bands throughout the Brillouin zone, with the states at the zone corners largely derived from the in-plane $5 d$ states of $e^{\prime}$ symmetry $\left(d_{x y}\right.$, $\left.d_{x^{2}-y^{2}}\right),{ }^{60}$ which thus have only weak interlayer hopping and thus limited dispersion. The zone-centre states, on the other hand, derive from the orbitals whose lobes point out of the $\mathrm{MX}_{2}$ plane $\left(a_{1}{ }^{\prime} d_{z^{2}}\right)$ orbitals, thus promoting increased interlayer hopping and a strongly three-dimensional character of the corresponding electronic states.

These effects also explain the broad appearance of the states at the zone centre in the measured spectrum shown in Figure 18a. As diucssed above, due to the intrinsic surface 
sensitivity of photoemission, it has a poor resolution in the out-of-plane momentum direction, thus leading to the broadening evident here for the electronic states which exhibit dispersion in this direction (i.e. the three-dimensional states). Despite the poor $k_{z}$ resolution, insight on the out-of-plane dispersions can still be obtained by performing measurements at different photon energies (Section 2.3), where measurements at different photon energies probe a Lorentzian distribution of $k_{z}$ values, but whose centre value varies with changing photon energy. Exploiting this, the above conclusions of dimenisonality of the underlying states have been validated explicitly, with photon-energy-dependent measurements indicating that the states at the zone centre and strongly dispersive along $k_{z}$, and are thus highly threedimensional, while those at the Brillouin zone corner exhibit essentially no out-of-plane dispersion with changing photon energy. ${ }^{60}$ The latter thus give rise to sharp spectral features as evident in Figure 18, as the poor out-of-plane resolution has no effect on states which do not disperse in that direction.

The multiple band extrema, which are close-by in energy and formed from states with different effective electronic dimensionality, underpin the transition between indirect and direct band gaps with reducing material thickness. In the bulk (Figure 18(a)), the band maximum at the $\bar{\Gamma}$-point is slightly higher than at the $\overline{\mathrm{K}}$-points, and thus the valence band maximum is formed from the three-dimensional states at $\Gamma$, with the two-dimensional states at $\mathrm{K}$ forming a secondary local maximum. A similar phenomenon occurs in the conduction bands, with the conduction band minimum formed by more three-dimensional states, in this case located away from a high-symmetry point, while two-dimensional states again form secondary band extrema at the zone-corner K-points. As the material is thinned down towards the monolayer limit, the states which are already strongly two-dimensional in character (and thus have their electronic wavefunctions largely localised within single layers of the structure) will be only little affected. The three-dimensional states, on the other hand, will experience a strong quantum confinement effect, pushing their band edges to higher energy. This is evident in measurements of thin $\mathrm{MoSe}_{2}$ films grown by molecular-beam epitaxy (Figure 18b,c, Ref. ${ }^{185}$ ). 
As the thickness is reduced, the states near to $\overline{\mathrm{K}}$ remain largely unchanged. In contrast, those at the Brillouin zone centre are pushed down in energy, with evident quantum well state creation: instead of a continuum of states there, individual defined states are observed, with a number corresponding to the number of $\mathrm{MX}_{2}$ layers.

A similar effect can be expected to occur for the conduction bands, again leaving the lowest-energy states as the two-dimensional states at the $\mathrm{K}$ points. Together with the valence band changes, this therefore drives the indirect-to-direct band gap crossover. To confirm that this is the case, however, it is necessary to also image the conduction band states. Besides bulk doping of the system, there are three ways in which this can be achieved. Exploiting the surface sensitivity of photoemission, a chemical dopant can be added at the surface, in order to dope the near-surface region of the material. This is commonly achieved by the deposition of small quantities (sub-monolayer) of alkali-metal atoms at the surface. Due to their low electronegativity, they will readily be ionized, donating their electrons into the near-surface region and thus achieving a tuneable electron doping up to on the order of $10^{14}$ carriers $/ \mathrm{cm}^{2}$ (e.g. Ref. ${ }^{186}$ ). In this way, the direct band gap of monolayer MoSe 2 and $\mathrm{MoS}_{2}$ thin films and exfoliated flakes have been confirmed via direct momentum-resolved ARPES measurements, ${ }^{185,187}$ and modifications of the electronic structure, such as due to carrier-mediated band-gap renormalization, ${ }^{186}$ have been observed. Care must be taken with this approach, however. The surface alkali atoms act as scattering centres, broadening the measured spectra. More importantly, they can intercalate into the material, form ordered superstructures, and can induce surface band-bending potentials. While these processes can in turn induce novel surface chemistry and physics, ${ }^{177,186,188,189}$ they can substantially complicates the interpretation of such data. Certainly, extreme care must be taken in interpreting the results of surface alkali-dosing as simply via a rigid shift of the chemical potential.

An alternative to chemical doping is photodoping. By illuminating the sample with an ultra-short laser pulse of above band-gap radiation, carriers can be optically excited across the band gap. ARPES measurements can then be performed with a second probe 
a

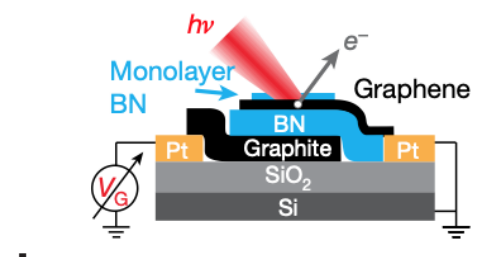

b
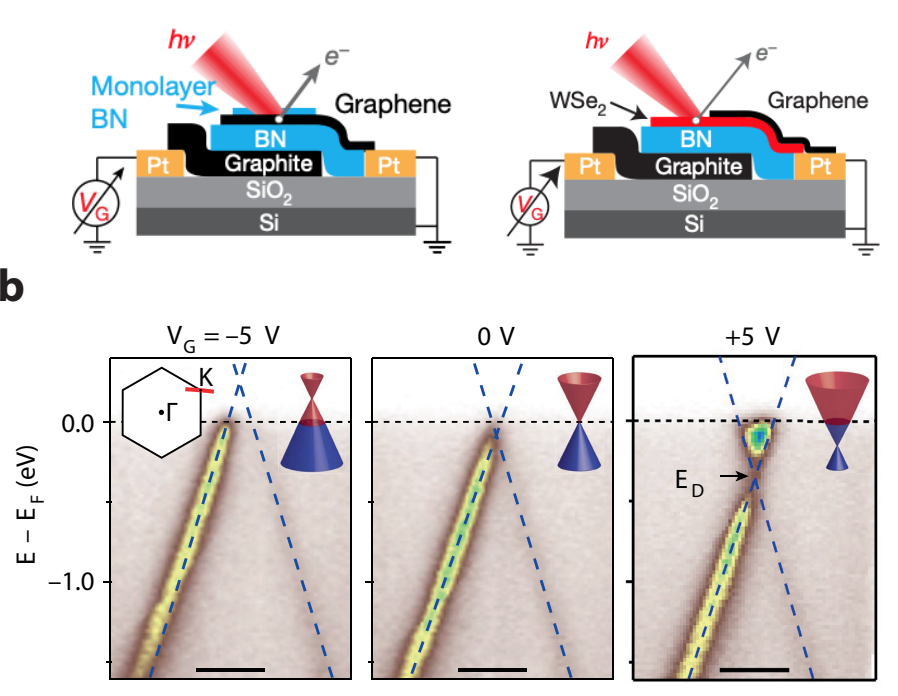

C

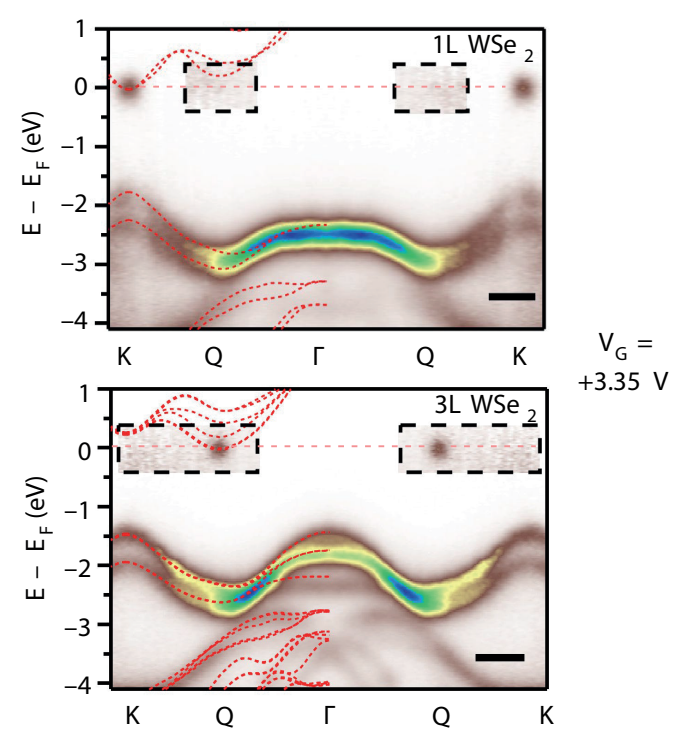

Figure 19: ARPES measurements with electrostatic gating. (a) Schematic of a 2D graphene (left) and $\mathrm{WSe}_{2}$ (right) heterostructure devices used for the ARPES studies. (b) ARPES measurements of the Dirac cone of graphene from the left device shown in (a), as a function of applied gate voltage. (c) Measurements of $1 \mathrm{ML}$ and $3 \mathrm{ML} \mathrm{WSe}_{2}$ under an applied gate voltage of $3.35 \mathrm{~V}$. Adapted with permission from Ref. ${ }^{194}$ Copyright 2019 Springer Nature.

pulse a short time later, before the photoexcited carriers have relaxed back to the valence band, allowing access to transiently photodoped states with carrier concentrations on the order of $10^{13} \mathrm{~cm}^{-2}$. While energy resolution is necessarily compromised as compared to ARPES measurements of occupied states, such time-resolved ARPES measurements provide much more than "just" a view of the nominally unoccupied band structure. By monitoring the ARPES signal at various time delays, excited state dynamics can be followed with momentum-space resolution, ${ }^{190-192}$ while carrier-induced band gap renormalization can be probed as a function of time delay, ${ }^{193}$ with the photoemission itself providing a simultaneous measure of the transient carrier population, electronic temperature and corresponding band structure changes.

A final method has recently been developed, which employs ARPES measurements simultaneously with electrostatic gating of samples (Figure 19). ${ }^{194}$ Ambipolar doping has been realised to date for graphene, while for monolayer TMDs, electrical injection of charge carri- 
ers into the conduction band has been achieved up to carrier concentrations of ca. $10^{13} \mathrm{~cm}^{-2}$. This is comparable to the carrier densities achieved using optical pumping approaches, although still around an order of magnitude lower than what can be achieved with surface doping, although it arguably provides a cleaner tuning parameter. As shown in Figure 19b, a clear electrostatic doping can be achieved using this method, while its application to fewlayer $\mathrm{WSe}_{2}$ provides another confirmation of the indirect-to-direct band gap crossover and to carrier-mediated band gap renoramlization effects discussed above (Figure 19c), in a device geometry that is comparable to that employed for measurements using other techniques (e.g. optical studies). ${ }^{194}$ While technically challenging, these measurements are now possible thanks to recent advances in both spatial-focussing of the light spot for ARPES, as well as the fabrication of device structures from exfoliated flakes. This provides an exciting opportunity for future studies of in operando devices, and for enabling direct comparisons between ARPES measurements and other studies on the same device structures.

As well as tuning semiconducting properties, the collective states of TMDs can also be expected to be sensitive to dimensional effects in the thin limit. Studies have, however, proved controversial, with varying claims of strengthening or suppression of different correlated orders, and the emergence of new ones, as samples transition from bulk to monolayer. An interesting example is $\mathrm{VSe}_{2}$. Despite its layered nature, photon energy-dependent soft X-ray ARPES measurements (Figure 20a) have successfully revealed a significant out-ofplane dispersion of the dominantly $\mathrm{V} 3 d$-electron derived bulk Fermi surface, pointing to significant interlayer hopping. In bulk form, $\mathrm{VSe}_{2}$ is known to host a weak-coupling charge density wave order. ${ }^{197,198}$ The ordering wavevector is again three-dimensional, and has been associated with a Fermi surface nesting, consistent with the three-dimensional character of the bulk Fermi surface (Figure 20b). Such a Fermi surface nesting will necessarily be modified as the system is thinned to the monolayer limit, and its electronic structure becomes strictly two-dimensional.

ARPES measurements of monolayer epitaxial $\mathrm{VSe}_{2}$ samples ${ }^{196,199,200}$ have shown how 

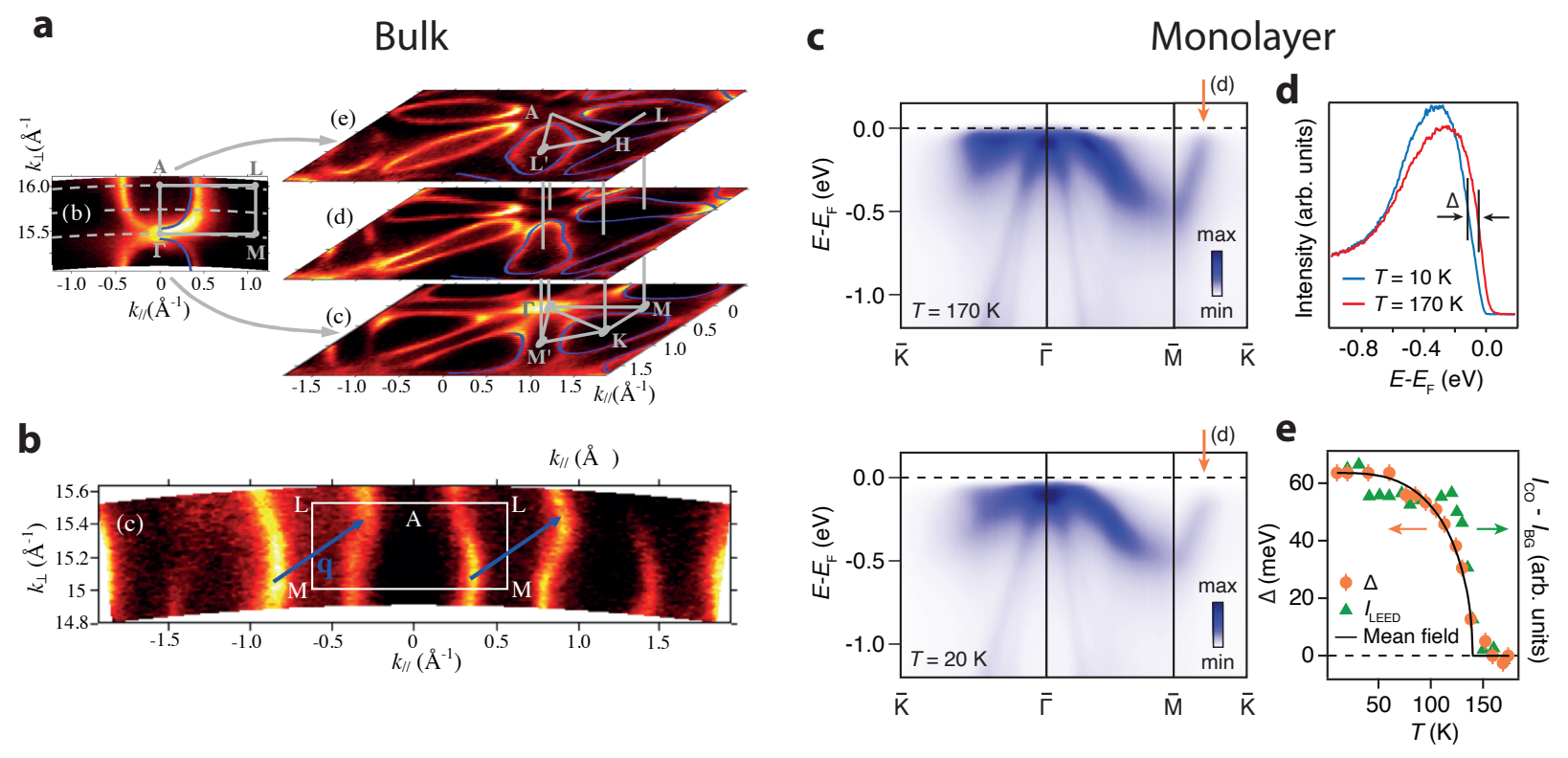

Figure 20: Soft X-ray ARPES measurements of bulk $\mathrm{VSe}_{2}$ showing (a) slices through the bulk Fermi surface and (b) the Fermi surface evolution in the plane of the CDW vector, with the nesting vector indicated. (a,b) Reproduced with permission from Ref. ${ }^{195}$ (c) ARPES measurements of MBE-grown monolayer $\mathrm{VSe}_{2}$ at $170 \mathrm{~K}$ (top) and $20 \mathrm{~K}$ (bottom), indicating the opening of a CDW gap as shown via energy-distribution curves in (d), and with the temperature-dependent gap in (e). (c-e) Aadapted with permission from Ref. ${ }^{196}$ Copyright (2018) American Chemical Society.

the CDW order not only persists, but is in fact strengthened as compared to the bulk. Figure 20b shows temperature-dependent measurements indicating the opening of a CDW gap at an enhanced transition temperature of $145 \mathrm{~K}$, as compared to $110 \mathrm{~K}$ in the bulk. ${ }^{196}$ More importantly, the energy gaps that open are considerably larger than in the bulk. Moreover, momentum-resolved measurements show how they extend across the full Brillouin zone, driving a metal-insulator-like transition. The direct measurements of these energy gaps from the ARPES allows one to estimate a value of $2 \Delta / k_{B} T_{c} \sim 10$, which places the CDW in monolayer $\mathrm{VSe}_{2}$ firmly in the strong-coupling limit. Volume-sensitive probes have claimed the presence of a robust (room-temperature) ferromagnetic state in monolayer $\mathrm{VSe}_{2}$, triggering much attention. ${ }^{201}$ This conclusion is not, however, supported by spectroscopic characterisation from ARPES - which shows an evident lack of the exchange splitting which would be expected to accompany such ferromagnetism. This conclusion is further supported by a lack of any 
X-ray magnetic circular dichroism, which is an elemental-specific probe of magnetism. ${ }^{196}$ Indeed, in contast to volumne-based studies, the spectroscopic studies indicates that ferromagnetism is not an intrinsic property of stoichiometric $\mathrm{VSe}_{2}$, although it can be induced via proximity-coupling with a neighbouring ferromagnetic layer. ${ }^{202,203}$ These results points to a particular advantage of ARPES and related surface-sensitive spectroscopic probes in characterising the collective states of $2 \mathrm{D}$ materials, where bulk-sensitive probes can more easily be complicated by extrinsic contamination effects in the substrate which dominate the volume of the measured sample.

\subsubsection{Spin-momentum locking}

It is not only for putative magnetic systems that the electron spin plays a crucial role in governing the properties of TMDs. Indeed, a number of TMD materials host spin-split electronic states, with the spin locked to the quasiparticle momentum, but with a momentum-space texture that ensures the system remains overall non-magnetic, similar to the Rashba effects discussed in Section 5.1.2. The visualisation of such momentum-dependent spin textures inherently require a probe that is both spin- and momentum-space sensitive. Spin-resolved ARPES provides exactly this capability, and as such has played a crucial role in uncovering non-trivial spin textures in TMDs.

An intriguing example is a so-called spin-valley-layer locking. ${ }^{146}$ As evident in Figure 14a, a single monolayer of the $2 \mathrm{H}$-type TMDs possesses no centre of inversion symmetry. In such a non-centrosymmetric environment, spin-orbit coupling will generically induce a momentumdependent lifting of the spin-degeneracy of electronic states. For the $4 d$ and $5 d$ transitionmetal based systems, spin-orbit coupling is strong, and significant effects can be expected. Xiao et al. ${ }^{150}$ showed how this leads to a pronounced spin-splitting developing particularly in the valence band states around the Brillouin zone corners in $\mathrm{MoS}_{2}$ and related group-IV TMDs. At these points, symmetry constrains the spin polarisation to be aligned normal to the monolayer, with a sign that reverses between neighbouring Brillouin zone corners 


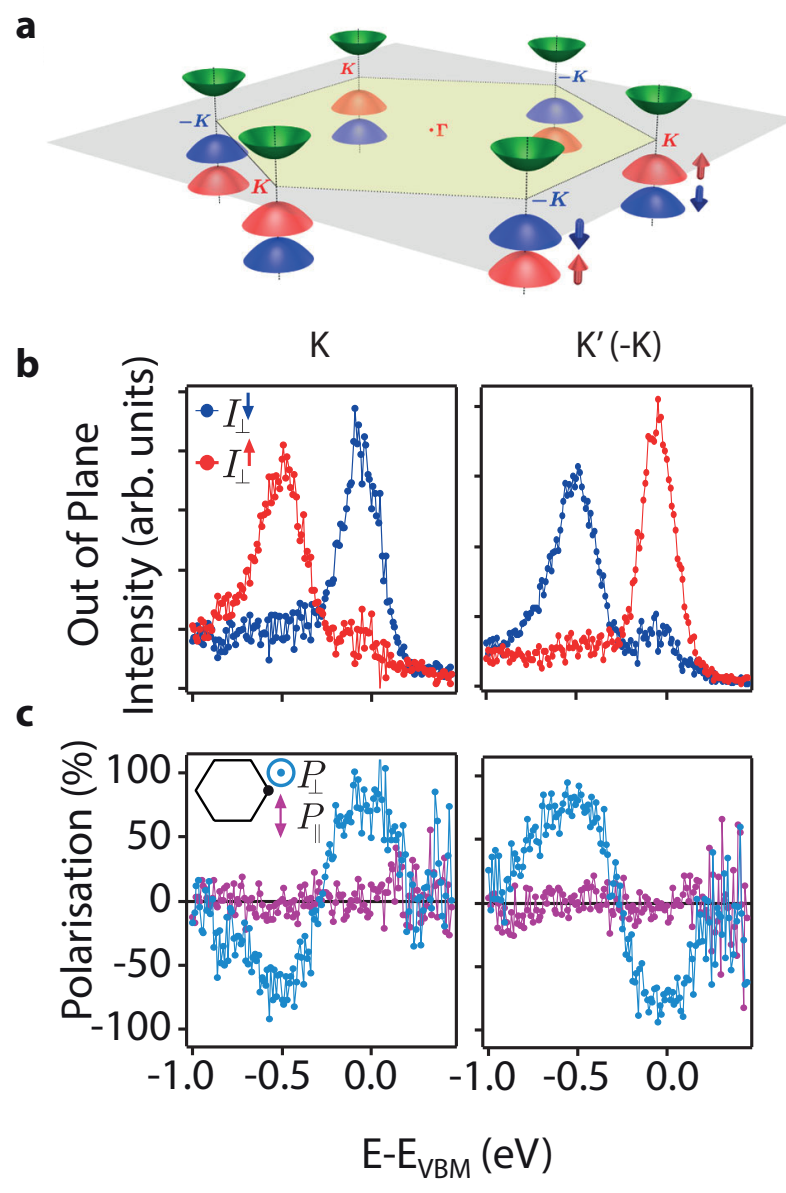

Figure 21: (a) Schematic of spin-valley coupled band-edge electronic structure of monolayer $\mathrm{MoS}_{2}$ and related group-IV TMDs. Reproduced with permission from Ref. ${ }^{150}$ (b) Spinresolved energy distribution curves measuring the out-of-plane component of the electron spin at the $K$ and $K^{\prime}$ points of bulk $\mathrm{WSe}_{2}$. (c) The corresponding out-of-plane spin polarisation is shown together with the in-plane polarisation extracted from complementary measurements. (b,c) Adapted with permission from Ref. ${ }^{60}$ Copyright 2014 Springer Nature.

(Figure 21a). This is termed a spin-valley locking, and is thought to underpin a host of novel optoelectronic properties of monolayer TMDs, including a pronounced circular polarisation of photoluminescence following photoexcitation, ${ }^{204,205}$ and a valley Hall effect controlled by circularly polarised optical excitation. ${ }^{206}$

Bulk 2H TMDs recover centrosymmetry, with a centre of inversion located between neighbouring $\mathrm{MX}_{2}$ monolayers which are stacked with $180^{\circ}$ relative rotation. The conventional expectation is that all spin-orbit driven spin splittings should vanish in this centrosymmetric environment. Nonetheless, spin-resolved ARPES measurements revealed strongly 
spin-polarised valence band states in bulk $2 \mathrm{H}-\mathrm{WSe}_{2}$ (Figure 21b). ${ }^{60}$ These measurements revealed a spin structure equivalent to the spin-valley locking expected in monolayer $\mathrm{MoS}_{2^{-}}$ type systems. Not only did this confirm the momentum-space spin texture characteristic of a spin-valley locking, but additional photon energy-dependent measurements combined with theoretical calculations indicated that the spin texture reverses from layer to layer in the crystal, representing a rich intertwining of momentum and real-space spin structures via a spin-valley-layer locking, ${ }^{146,207}$ and a novel route by which local inversion symmetry breaking (i.e. at the level of the point group) is able to generate spin-split electronic states even for a system with a centrosymmetric space group. ${ }^{208}$ The observation of such spin-polarised electronic states by ARPES exploits the extreme surface sensitivity of the technique, allowing it to be most sensitive to the topmost layer-localised spin structure. Subsequent measurements have utilised this effect to probe the influence of spin-valley coupling on intervalley relaxation effects using pump-probe ARPES, ${ }^{191}$ have shown that such spin-valley coupling can be observed in the monolayer limit, ${ }^{209}$ and have shown that a similar spin-valley locking is present in the Fermi surface sheets of the group $\mathrm{V}$ metals, ${ }^{210}$ where it underpins the stabilisation of an unusual so-called Ising superconducting state which is remarkably resiliant against application of in-plane magnetic fields. ${ }^{211}$

As discussed in more detail in Section 5.1.1, momentum-dependent spin textures are also a characteristic signature of non-trivial topological order via the formation of spin-helical Dirac cone surface states. A rather general route for generating such states was found for the $1 \mathrm{~T}$ and $2 \mathrm{H}$ family of TMDs, ${ }^{155,212}$ as shown in Figure 22 . The chalcogen sites of the TMDs experience a trigonal crystal field. This splits their $p$-orbital energy levels into a $p_{z}$ state and a $p_{x / y}$ doublet, which is further split by spin-orbit coupling. As there are two atoms per $\mathrm{MX}_{2}$ unit, bonding and anti-bonding combinations are formed, allowing the possibility to generate even and odd-parity states. The $p_{z}$ states have their lobes pointing between the layers, and so naturally experience higher inter-layer hopping than for the $p_{x / z}$ states, leading to a larger out-of-plane dispersion of the $p_{z}$-derived states. Consequently, as long 
a

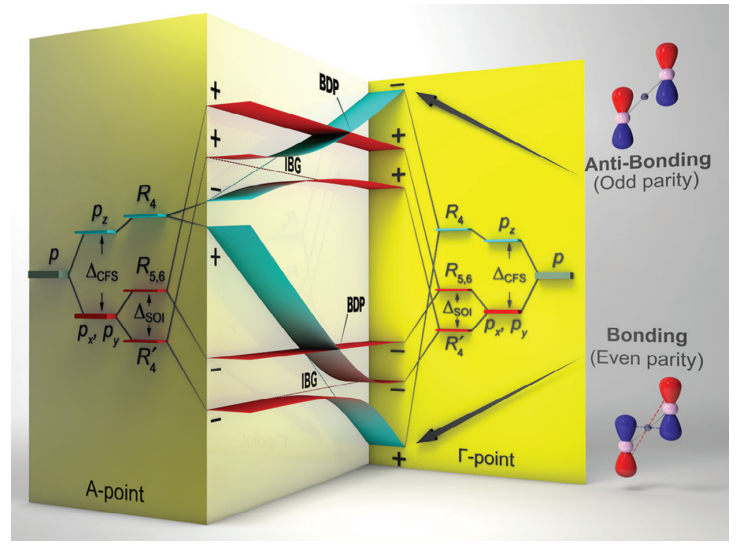

b

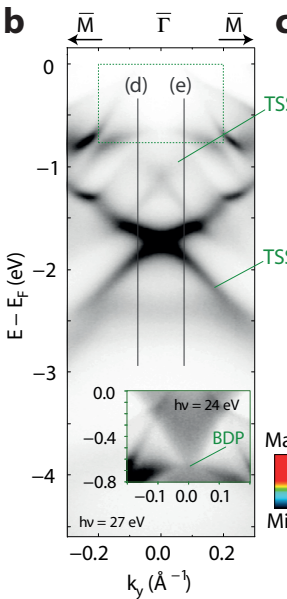

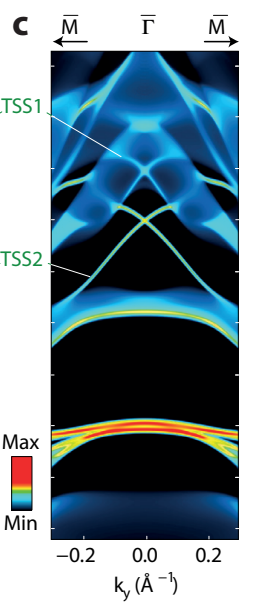

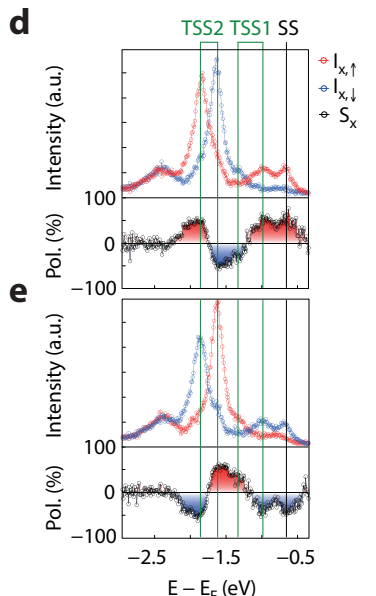

Figure 22: (a) Schematic of influence of bonding, crystal field splitting, spin-orbit coupling and out-of-plane dispersion of $p$-orbital states in TMDs, showing the formation of protected bulk Dirac points and topologically non-trivial inverted band gaps. Reproduced with permission from Ref. ${ }^{212}$ (b) ARPES measurements and (c) corresponding supercell calculations from density-functional theory of the occupied electronic structure of $\mathrm{PdTe}_{2}$, showing the formation of topological surface states within the invertd band gap, and the formation of a bulk Dirac cone (see inset). (d,e) Spin-resolved EDCs measured along the cuts indicated in (b), showing the spin texture characteristic of a ladder of topological surface states. (b-d) Adapted with permission from Ref. ${ }^{155}$ Copyright 2017 Springer Nature.

as the interlayer hopping is larger than the crystal-field splitting, the $p_{z}$-derived states will cross through the $p_{x / y}$ pair as a function of out-of-plane momentum.

Photon energy-dependent ARPES measurements have shown how some of these crossings remain protected, forming three-dimensional bulk Dirac cones, while others hybridise, generating parity-inverted band gaps which host topological surface states and surface resonances . ${ }^{55,212-220}$ An example of this is shown for $\mathrm{PdTe}_{2}$ in Figure 22b), where the above ladder of topological states is found, in good agreement with density-functional calculations (e.g. Figure 22c). Spin-resolved ARPES has been utilised to probe the spin texture of the resulting surface states, to confirm its topological origin (Figure 22d) and to disentangle the complex surface electronic structure of these systems. This mechanism has been shown to be generic to the $2 \mathrm{H}$ and $1 \mathrm{~T}$ TMDs, ${ }^{155}$ and has been experimentally observed in at least 8 separate TMDs to date. This therefore represents a particularly flexible material system in which to probe topological physics, and to probe the interplay with the wide variety of other orders 

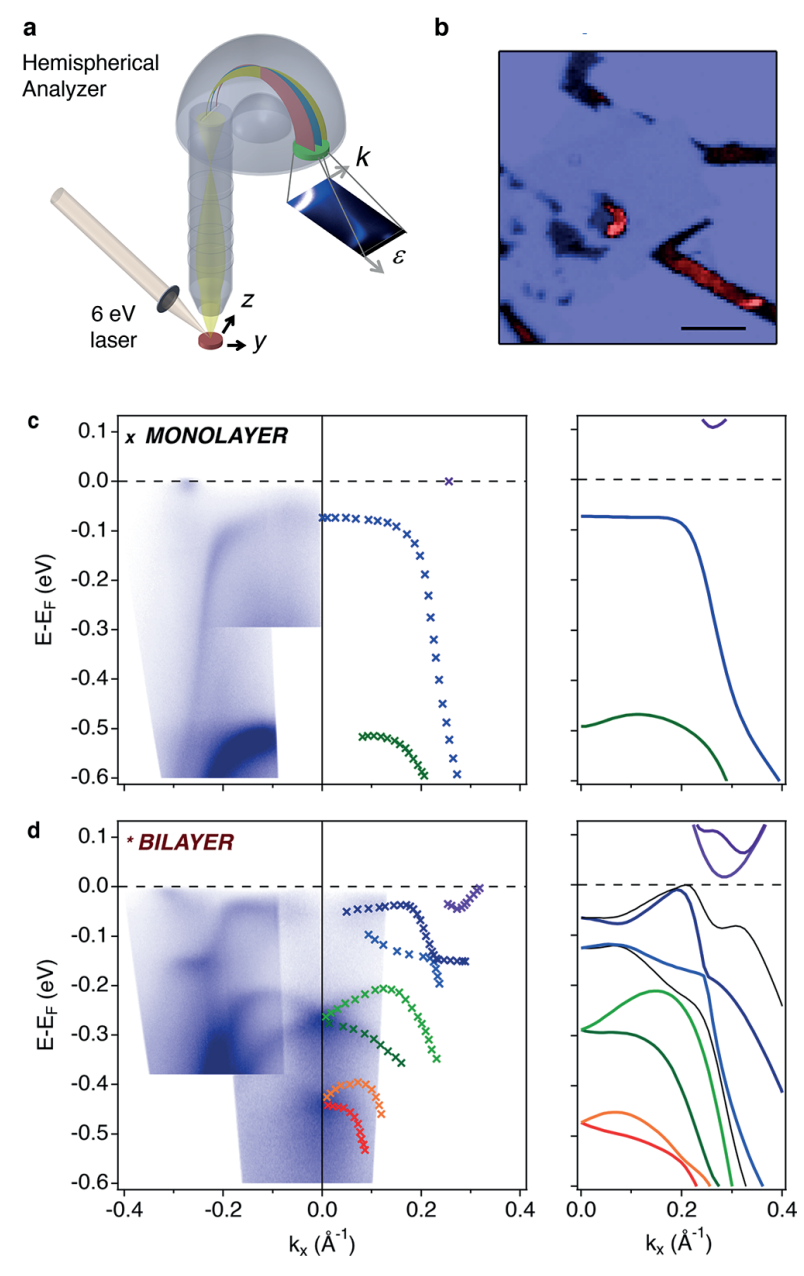

Figure 23: (a)Schematic of focused laser $\mu$-ARPES setup and (b) real-space $\mu$-ARPES intensity map (scale bar: $100 \mu \mathrm{m}$ ). (c,d) $\mu$-ARPES measurements and extracted band dispersions (left) and DFT calculations (right) of monolyaer (c) and bilayer (d) 1T'-WTe 2 . Adapted with permission from Ref. ${ }^{221}$ Copyright (2019) American Chemical Society.

which this materials family hosts. Their discovery again reflects the power of utilising the momentum-space and spin- sensitive probe of ARPES together with state-of-the-art density functional theory in order to disentangle the underlying origins of the topological behaviour in solids, and the possibilities to gain chemical control over this.

As well as for the $2 \mathrm{H}$ and $1 \mathrm{~T}$ TMDs, the distorted $1 \mathrm{~T}^{\prime}$ structures have been intensely investigated for their topological properties, as potential hosts of Weyl fermions with so-called type-II Weyl points. ${ }^{154}$ Their electronic structure is predicted to show a strong evolution with materials thickness. Thin-film samples have been investigated by ARPES using a combined 
MBE-ARPES approach, ${ }^{222}$ but suffer from the occurrence of multiple structural domains with different orientations within the probing light spot area. Cucchi et al. ${ }^{221}$ have recently demonstrated a focussed laser approach, where they are able to resolve separately the electronic states corresponding to bilayer and monolayer patches of exfoliated and encapsulated flakes of $1 \mathrm{~T}^{\prime}-\mathrm{WTe}_{2}$ (Figure 23). A clear transition is observed from a gapped state in the monolayer (with weak occupation of the bottom of the conduction band), to a system with negligible band gap but band splittings characteristic of Rashba-type interactions, representing a broken inversion symmetry, in the bilayer. Crucially, the observation of such fine features, particularly around the Fermi level, rely both on the preparation of high-quality samples with suitable encapsulation, but also the high energy and momentum resolutions afforded by laser-based $\mu$-ARPES as compared to synchrotron-based approahces for nanoARPES. While this is accompanied by limited momentum-space range and poorer spatial resolution than can be achieved at synchrotrons, such laser-based approaches are likely to find increasing application in the study of mesoscale systems in the coming years, and to be expanded to enable, e.g., spin-resolved or time-resolved measurements to also be performed from small sample regions.

\subsection{Transition metal Oxides}

Transition metal oxides (TMOs) are a challenge for both chemistry and physics. ${ }^{223-225}$ Their extemely rich phase diagrams span across a wide spectrum of magnetic and electronic properties, and the comparable energy scale of their bandwidth with electron correlations make them ideal systems to explore the new landscape of quantum material 'properties on demand' via advanced parameters of growth (e.g. doping, heterostructures, strain) and methods of synthesis. ${ }^{226-228}$ Quantum properties provide many opportunities to engineer unprecedented electronic states in TMOs, and may lead to novel properties and functionalities. Unravelling what ultimately drives the electronic and magnetic properties in TMOs is strictly linked to a direct and detailed measurement of the electronic energy distribution of the metal orbitals. 

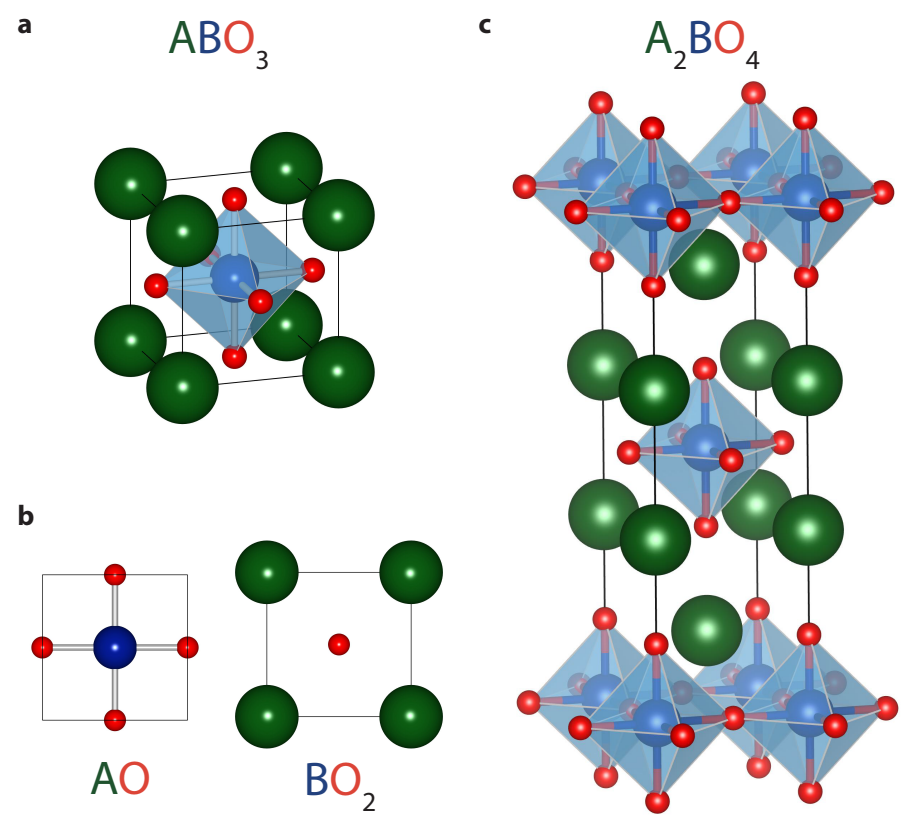

Figure 24: Crystal structure of cubic and layered perovskite TMOs. (a) Cubic $\mathrm{ABO}_{3}$ systems, comprised of alternating stacks of (b) $\mathrm{AO}$ and $\mathrm{BO}_{2}$ planes. (c) In the layered $\mathrm{A}_{2} \mathrm{BO}_{4}$ structure, perovskite $\mathrm{ABO}_{3}$ blocks are separated by rocksalt $\mathrm{AO}$ blocks, with weak bonds between neighbouring AO planes making this a natural cleavage plane, rendering such systems particularly suitable for ARPES measurements.

Here PES plays an important role, being (i) a local probe sensitive to the atom environment, (ii) able to resolve different oxidation states and (iii) able to disentangle and to follow vs external parameters (e.g. temperature) the presence of mixed valence and electronic correlation. ${ }^{18,27,229,230}$

Some of the most common TMOs are based around the parent crystal structure of strictly cubic $\mathrm{ABO}_{3}$ perovskites (space group $\mathrm{Pm}^{-} 3 \mathrm{~m}$ ), shown in Figure 24. The B cations are 6coordinate with the neighbouring oxygen atoms in an octahedral arrangement, while the larger A cations are 12-coordinate. The structure can alternatively be described in terms of alternating. $\mathrm{BO}_{2}$ and $\mathrm{AO}$ layers. The valencies of $\mathrm{A}$ and $\mathrm{B}, V_{A}$ and $V_{B}$, must satisfy a sum rule $V_{A}+V_{B}=6$. When $V_{A}=2$ and $V_{B}=4$ the $\mathrm{AO}$ and $\mathrm{BO}_{2}$ layers are each charge neutral. Layered perovskite variants exist. For example, the so-called single-layered compound of general formula $\mathrm{A}_{2} \mathrm{BO}_{4}$ involve a layer sequence $(\mathrm{AO})(\mathrm{AO})\left(\mathrm{BO}_{2}\right)(\mathrm{AO})(\mathrm{AO})\left(\mathrm{BO}_{2}\right)$ etc., while intermediate Ruddleston-Popper phases involve both $(\mathrm{AO})(\mathrm{AO})$ bilayers and $(\mathrm{AO})$ monolay- 
ers, thus giving a rich structural diversity. Both distortions or tilting of the octahedron often occurs, leading to an almost limitless phase diagram with a large number of physical and chemical properties, spanning from the well known high $T_{c}$ superconductivity in cuprates and giant magnetoresistance in manganites to multiferroicity.

The geometrical arrangement, the occupancy of the transition metal $3 d$ orbitals and their hybridization (or Coulomb repulsion) with $p$ electronic states of oxygen are the main reason of their diversity. Consequently, the last two decades have seen a huge amount of research devoted to perovskite-based TMOs, where the limit of the most advanced growth techniques was pushed forward in producing heterostructures with specific properties. Both characterization and theoretical analysis were challenged by the two extrema of interpretation: on the one hand the so called 'ionic-limit', where hybridization is not considered, and on the other hand the number of electrons involved in the band and the ability of electrons to travel in the solid. Photoemission probes the excited state of a solid, i..e the solid in presence of a hole, and collective effects has been of paramount importance in the understanding of the complex physics of TMOs, where both cations and anions in their ground state may have very different valence from their formal oxidation state. A general approach to this area is provided by the Zaanen, Allen, Sawatzky model, which provides a basis for distinguishing between metallic and non metallic transition metal perovskites oxides. ${ }^{231,232}$

\subsubsection{In-gap and extended states at $E_{f}$}

Looking at catalytic properties, the large diversity of observation in TMOs can be often explained in terms of the behavior of photoexcited carriers both in the bulk and at the surface of these materials. ${ }^{233-236}$ In particular the role of oxygen vacancies has been strongly debated in the last decade. ${ }^{40,45,46,237}$ In fact, important changes in the electronic structure have been observed as induced by the chemical doping arising from the formation of oxygen vacancies; an oxygen vacancy can act as a two electron donor and effects such as the formation of in-gap defect states, electron accumulation and surface band bending have been linked to vacancies. 
A further relevant observation often found at the surface of TMOs with a large band gap (or at their interface with other TMOs) is that the introduction of carriers into localized energy levels within the band gap (e.g. by oxygen vacancies and/or cation off-stoichiometry) often produces both the stabilization of a dispersive bi-dimensional electron gas (2DEG) at the surfaces, and the formation of localized non-dispersive states, so called 'defect' in-gap (IG) states. ${ }^{39,45,48,238-241}$ Starting from the first observation of 2DEG at the LAO/STO interface and at STO single crystals, a rich variety of such states have been reported both in parent materials and in engineered heterostructures. ${ }^{47,48,242}$ Different behavior and character are found: some analyses reveal that in STO these states are deep-level traps associated with defects resulting from interstitial oxygen suggesting defect interconversion, ${ }^{41}$ while other reports highlight in LAO/STO the importance of acceptors and donors states as well as the difference between crystalline and amorphous systems. ${ }^{238}$ It has been now made clear, both theoretically and experimentally, that understanding and controlling the behavior of oxygen vacancies and excess electrons is essential to improve performances for future applications.

It is important to mention that the use of tuneable synchrotron radiation in combination with ARPES provides a valuable tool for disentangling the electronic contribution in the valence band of oxides. By using resonant PES in the valence band, i.e., measuring the valence band PES of a system while varying the photon energy across an absorption edge, one is able to obtain not only information about atoms with a localized vs. delocalized electronic character, but also to discriminate two oxidation state in the same atomic species, in the case of mixed valence compounds, i.e. obtain site-selective information. ${ }^{46,243-245}$ In recent years, soft X-ray ARPES and resonant ARPES have been developed at large scale facilities; thanks to the increased flux and energy resolution of modern beamlines, identification of both itinerant and localized character in TMOs and analysis of buried interface have been possible. We refer the reader to detailed review papers and books on these specific experimental methodologies. ${ }^{244}$ 

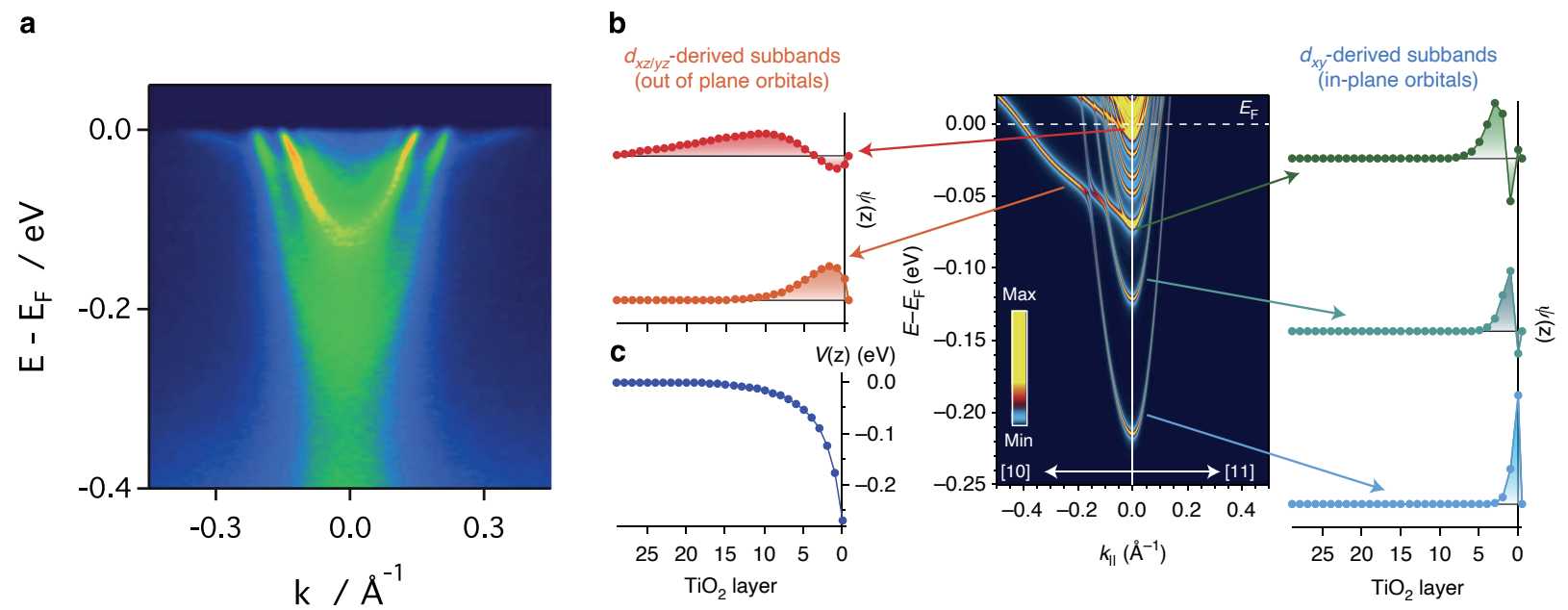

Figure 25: (a) Measured surface electronic structure of a 2DEG formed on the (001) surface of $\mathrm{SrTiO}_{3}$. Reproduced with permission from Ref. ${ }^{246}$ Copyright 2015 Wiley-VCH. (b) Corresponding self-consistent tight-binding supercell calculation. The spatial dependence of the subband wavefunctions along the confinement direction, $\Psi(z)$, reveal a pronounced realspace orbital ordering as a direct consequence of near-surface band bending (potential shown in $(c))$. (b,c) reproduced with permission from Ref. ${ }^{247}$ Copyright 2014 Springer Nature.

\subsection{2 $\quad \mathrm{SrTiO}_{3}$}

Due to the surface sensitivity of ARPES, studying the buried 2DEG at the LAO/STO interface is challenging. There have been several pioneering reports, in particular exploiting resonant enhancement of photoemission from the Ti $3 d$-orbital states to make it possible to probe the 2DEG and in-gap states below the LAO top layer. ${ }^{248,249}$ In addition, the discovery that the 2DEG can also be formed at the bare $\mathrm{SrTiO}_{3}$ surface, ${ }^{250,251}$ has also opened possibilities for high-resolution measurements of the electronic structure of the $\mathrm{SrTiO}_{3}$ 2DEG states. Figure 25a shows ARPES measurements of the 2DEG states in a high carrier-density 2DEG induced by oxygen vacancy creation at a cleaved $\mathrm{SrTiO}_{3}$ surface, with the oxygen vacancies induced by exposure to the synchrotron beam. Several points of note are immediately obvious from inspection of the measured dispersion. First, multiple electronic states can be resolved, separated in energy. Secondly, states of very different effective masses are observed, with ladders of light bands having relatively large bandwidths, and an additional state with much heavier effective mass being located closer to the Fermi level. All of these features can 
be attributed to the effects of quantum confinement in the near-surface quantum well which defines the 2DEG (Figure 25c). In a nominal $d^{0}$ configuration, the unoccupied conduction band of stoichiometric $\mathrm{SrTiO}_{3}$ is composed of Ti $d$-orbitals. Apart from very small energetic splittings due to spin-orbit coupling and the effects of a tetragonal structural distortion, the three $t_{2 g}$ orbitals $\left(d_{x y}, d_{x z}, d_{y z}\right)$ are degenerate at the bottom of the conduction band in bulk $\mathrm{SrTiO}_{3}$. However, when subjected to a near-surface band bending, the states corresponding to $d_{x z}$ and $d_{y z}$ orbitals whose lobes point out of the plane (i.e. along the confinement direction) "feel" the effects of the confining potential most pronouncedly, being pushed upwards in energy relative to the in-plane $d_{x y}$ orbitals (Figure 25b). In contrast, the $d_{x y}$-derived states "sit down" in the resulting quantum well, leading to a set of subband states that are reminiscent of the ladder of quantum well states familiar from semiconductor quantum wells. The result is a creation of an effective orbital ordering with respect to the bulk system, and the formation of the complex multi-subband structure of both heavy and light states shown in Figure 25.

ARPES has been used to identify the orbital character of these states from intensity differences arising from transition-matrix element variations when measured using light linearly polarized in both vertical and horizontal planes, ${ }^{250}$ finding orbital assignments in line with the above considerations. Moreover, the ARPES band structure gives a direct measure of the near-surface carrier density from the area of the corresponding Fermi surfaces; a quantity which is challenging to determine from other measurements given the surface-localised and multi-band nature of this system, and allows correlating the changes in higher-energy features (in particular a shift of the valence band to higher energies and the development of in-gap states) with the formation of the 2DEG states at the Fermi level. This has been exploited to probe the underlying origin of the 2DEG observed at the surface of $\mathrm{SrTiO}_{3}$. In particular, with exposure to atomic oxygen, the valence band was observed to shift back to lower binding energies, the in-gap states to be largely quenched, and the 2DEG states to vanish. This indicates that the origin of the surface 2DEG is indeed the creation of 

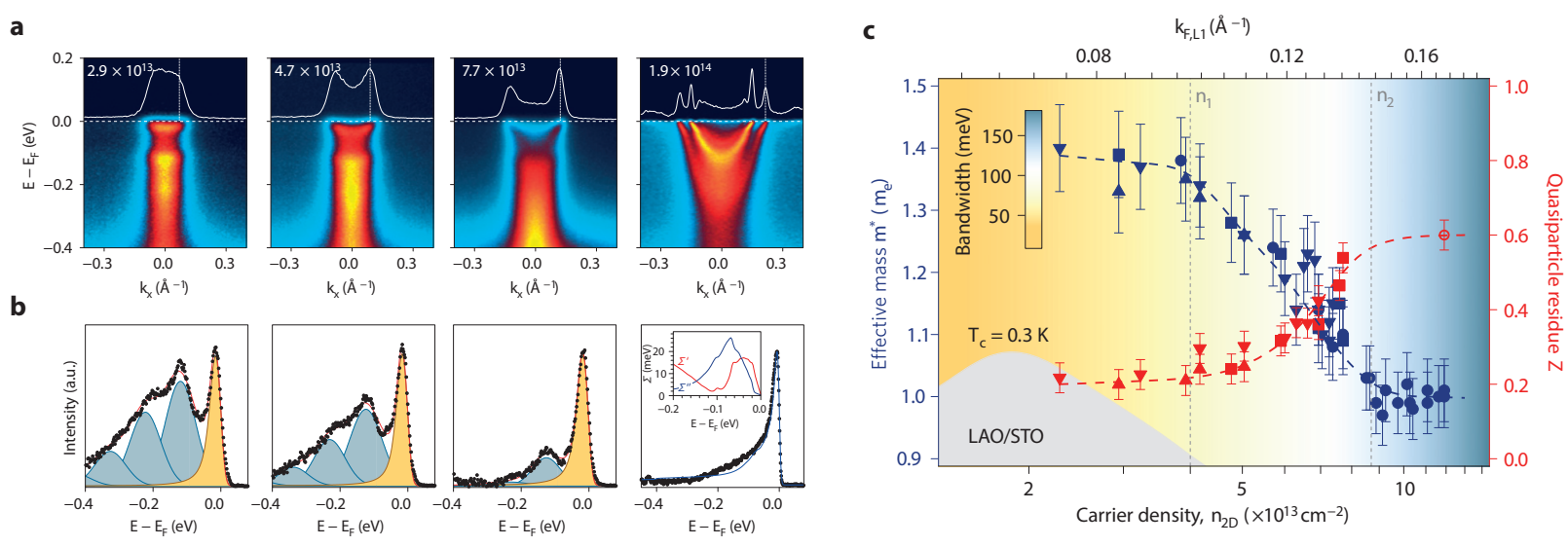

Figure 26: (a) ARPES measurements as a function of increasing surface carrier density of the surface 2DEG of $\mathrm{SrTiO}_{3}$. (b) Corresponding energy distribution curves showing the evolution of a satellite structure indicative of polaron formation. (c) Extracted effective mass and quasiparticle-residue extracted from the ARPES measurements, overlaid on a schematic phasse diagram of the LAO/STO interface 2DEG system. All panels adapted with permission from Ref. ${ }^{254}$ Copyright 2016 Springer Nature.

oxygen vacancies, largely induced by exposure to the synchrotron beam during the ARPES experiment. ${ }^{246,251-253}$

Beyond simply identifying the basic band structure of the $\mathrm{SrTiO}_{3} 2 \mathrm{DEG}$, however, ARPES also provides valuable insights into the nature of electronic interactions in the resulting electron liquid. Already in Figure 25a, a decrease in the slope of the rapidly dispersing states is evident in the vicinity of the Fermi level (a so-called "kink" in the dispersion). Quantitative analysis ${ }^{247}$ has shown that this arises due to a moderately-strong electron-phonon coupling. Interestingly, the strength and nature of the electron-phonon coupling changes markedly with varying carrier density of the 2 DEG states. ${ }^{254,255}$ In the low carrier density limit (i.e. for 2DEG states corresponding to a smaller surface carrier density), a pronounced satellite structure is observed in the measured electronic structure (Figure 26a,b, composed of replica bands separated from the lowest-energy band with a spacing of $100 \mathrm{meV} \cdot{ }^{254-256}$ This is equal to the longitudinal optical phonon mode frequency, and the form of the satellite structure indicates the formation of polarons driven by a long-range Fröhlich coupling to a single phonon mode. Similar replica features have been observed in other transition-metal 
oxides including $\mathrm{Sr}_{2} \mathrm{TiO}_{4},{ }^{257} \mathrm{TiO}_{2},{ }^{258}$ and $\mathrm{EuO},{ }^{259}$ indicating that they are a likely a generic feature of lightly-doped polar oxides. With increasing carrier density, the preferential coupling to the single LO mode becomes weaker, and the electron-phonon coupling thus becomes weaker and more short-ranged, leading to the loss of the defined satellites, and the emergence of the near- $E_{F}$ "kink" structure disucssed above (Figure 26a,b). First-principles calculations including electron-phonon coupling have shown how this occurs due to increased screening of the long-range electron-phonon interaction as the plasma frequencuy becomes greater than the mode energy of the LO phonon with increasing doping. ${ }^{260}$ Additional experiments and calculations on doped $\mathrm{EuO}$ have also identified how polaron features can arise due to coupling of the free charge carriers with the plasmon modes of the electron gas themselves, ${ }^{259}$ and it seems likely that similar features may also be present for the $\mathrm{SrTiO}_{3} 2 \mathrm{DEG}$, although they have not been resolved to date.

The determination of the role of these many-body effects on the electronic structure of the 2DEG provides important insights for interpreting the origin of other physical properties of the interacting electron system. For example, the 2DEG at the STO/LAO interface is known to exhibit a dome of superconductivity with increasing carrier doping. Quantitative extraction of the effective mass and quasiparticle residue, encoding the strength of the electron-phonon coupling, from the measured ARPES data of the surface 2DEG of $\mathrm{SrTiO}_{3}$ are shown in Figure 26. They highlight how the polaronic regime may be intricately connected to the occurance of superconductivity, and highlight how the complex evolution of electron-phonon coupling with carrier doping in this system may play a key role in controlling the doping-dependent phase diagram of this system.

\subsubsection{Anatase $\mathrm{TiO}_{2}$}

A related system, titanium dioxide $\mathrm{TiO}_{2}$, has three main polymorphs, namely rutile, anatase and brookite, and has aroused ongoing interest over many years as a photocatalyst, in processes such as water splitting. ${ }^{236,261,262}$ In the specific case of anatase $\mathrm{TiO}_{2}$, an insulator 
with a $3.2 \mathrm{eV}$ band-gap, one again observes under specific conditions of growth and/or photoirradiation the presence of both non-dispersive in-gap states (IG) as well as dispersive 2DEG electronic states near the Fermi level. ${ }^{39,45,238}$ A number of issues remain to be resolved, including the relationship between the 2DEG and localised IG states and how best to control their formation and quenching. PES and ARPES give important information on these matters. Figure 27 summarizes the electronic properties along the $\Gamma-X$ direction of the surface projected Brillouin zone (BZ) for an optimal anatase (001) film. Dispersive and sharp features are detected in the valence band from $4 \mathrm{eV}$ to $8 \mathrm{eV}$ binding energies (right panel ), consistent with the $3.2 \mathrm{eV}$ energy band gap value expected. Nevertheless, additional spectral weight is present within the energy gap, as clearly shown by the zoom of the integrated DOS and by the corresponding ARPES spectrum (top-left ). Two features are readily identified: the broad, non-dispersive, IG state located at $E_{B} 1.6 \mathrm{eV}$ and a dispersive metallic 2DEG reaching the Fermi energy. Panel (c) in Figure 27 displays the parabolic dispersion of the 2DEG as measured in the 2nd BZ. In this case, no suppression of intensity due to symmetry rules is observed (see Figure 4 and Appendix A). An inner replica, arising from lateral confinements of a surface reconstruction, is also visible. ${ }^{46}$ The parabolic fit of the two metallic states is also reported as red-dashed lines on the left-side of the ARPES spectrum.

A further interesting aspect is the connection (or not) between IG and 2DEG: while in $\mathrm{SrTiO}_{3}$, oxygen vacancies provide electrons to both the IG and 2DEG states, the different reactivity of specific oxygen sites (surface or subsurface) may lead to 2DEG formation with negligible amount of localized IG-states, or stabilization of IG-states alone. ${ }^{39,238}$ In the case of $\mathrm{TiO}_{2}$, one observes variation of the energy position and an evolution vs. annealing temperature. Valence band PES spectra in Figure 28 show that IG states significantly change as a function of the substrate used for the growth, a behavior often found in other large band gap TMOs. ${ }^{239}$ The stabilisation of the 2DEG dispersive state is however not affected by the overall structure, as one observes the presence of states close to the Fermi energy 
a)

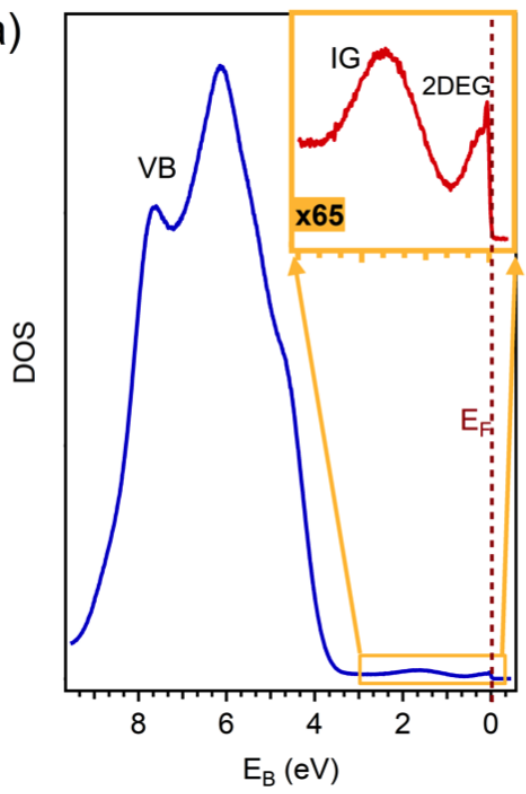

b)

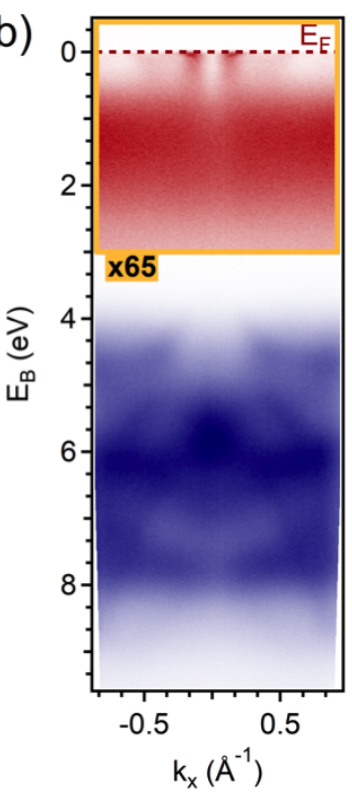

C)

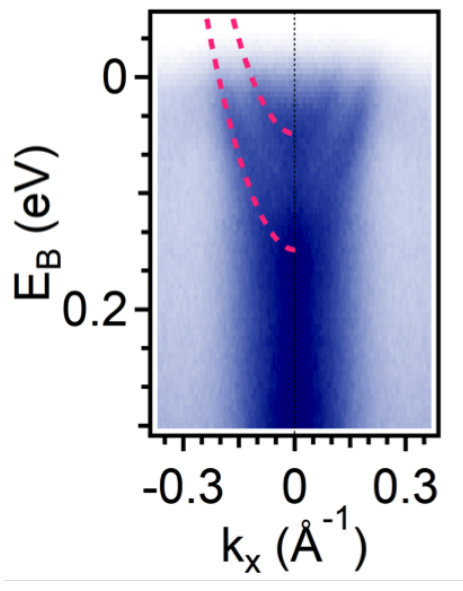

Figure 27: ARPES band dispersion of an optimised anatase $\mathrm{TiO}_{2} / \mathrm{LaNiO}_{3} / \mathrm{LaAlO}_{3}$ thin film. Spectra have been acquired at $\mathrm{hv}=46 \mathrm{eV}$ and horizontal polarisation. a): valence band density of states (DOS); inset: zoom ( 65 times) of the zone close to the Fermi level, showing the localised IG and the metallic 2DEG. Panel b), ARPES spectra of panle a): the non-dispersive IG state corresponds to the flat broad states locate between $1 \mathrm{eV}$ and $2 \mathrm{eV}$ of binding energies. Conversely, the 2DEG is characterised by parabolic electron-like dispersion obeying $t 2 g$ symmetry. c) the parabolic dispersion of the 2DEG as measured in the 2 nd BZ. The parabolic fit of the two metallic states is also reported as red-dashed lines on the left-side.

irrespective of the specific substrate, albeit the sharpest peak is measured for the sample grown with minimum lattice mismatch (i.e. $\mathrm{LaAlO}_{3}$ ). In general, defect states created by oxygen vacancies as IG and 2DEG may have different electronic character, and also in $\mathrm{TiO}_{2}$ such difference may be highlighted by resonant-ARPES. Angular resolved resonant ARPES experiments reveal that 2DEG features and non-dispersive states located at 1 ev of BE resonate at different photon energies, corresponding respectively to $e_{g}$ and $T i^{3+}$ sites: ${ }^{39,263,264}$ this suggests that only the latter state is strongly localized in correspondence of oxygen vacancies and can be considered an impurity state. ${ }^{238,265}$ 

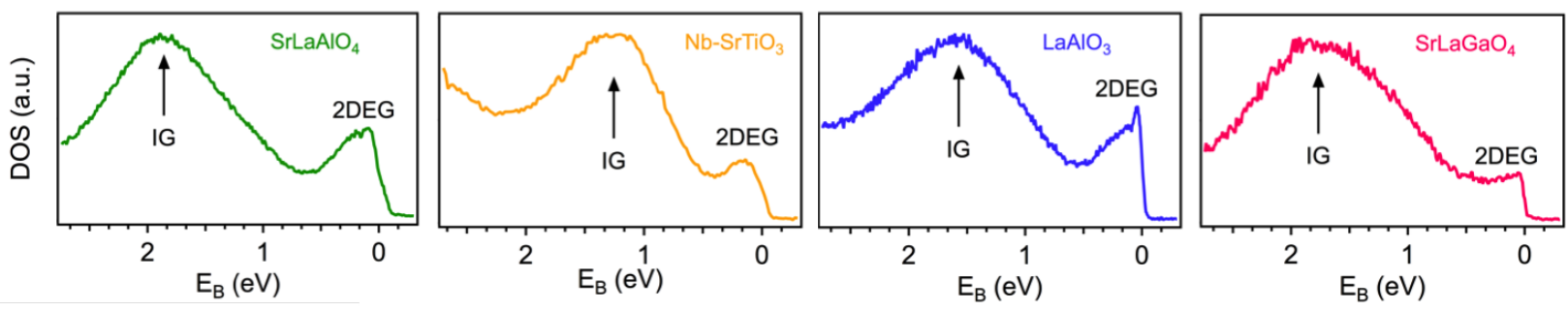

Figure 28: Photoemission Spectra of the DOS in the vicinity of the Fermi level for $\mathrm{TiO}_{2}$ anatase films grown on different substrates, measured at $\mathrm{hv}=46 \mathrm{eV}$. The energy position of the IG states varies and the fine structure of the 2DEG is revealed, as marked by the arrows.

\subsubsection{Metallic oxides}

The previous sections discussed studies of nominally insulating three-dimensional oxides, where metallic two-dimensional states were created at their surfaces. ARPES has also found extensive application in the study of bulk oxide metals with a quasi-two-dimensional layered structure. Perhaps most famously, it has been instrumental in establishing the Fermiology, superconducting gap structure, and doping-dependent phase diagrams of the hightemperature cuprate superconductors. As extensive reviews have been written on this topic (e.g. Refs. ${ }^{14,266-268}$ ) we do not treat this further here. We will, however, discuss here a different layered oxide, $\mathrm{Sr}_{2} \mathrm{RuO}_{4}$, which has served as a benchmark material for establishing the validity of photoemission as a bona fide probe of the interacting electronic structures of solids.

$\mathrm{Sr}_{2} \mathrm{RuO}_{4}$ is a textbook Fermi liquid below $T \approx 25 \mathrm{~K},{ }^{269}$ with quantum oscillation measurements indicating substantial effective mass enhancements as a result of electronic correlations, ${ }^{269,270}$ while it has been extensively investigated as a putative unconventional supercon-

ductor. ${ }^{271,272}$ Despite a pronounced surface reconstruction complicating the interpretation of photoemission spectra from pristine samples, it has now been firmly established that surface treatments can be utilised to suppress ARPES intensity coming from the surface states, with the spectra instead dominated by the bulk states. ${ }^{273}$

Figure 29a shows the Fermi surface as measured in a recent laser-ARPES experiment, 

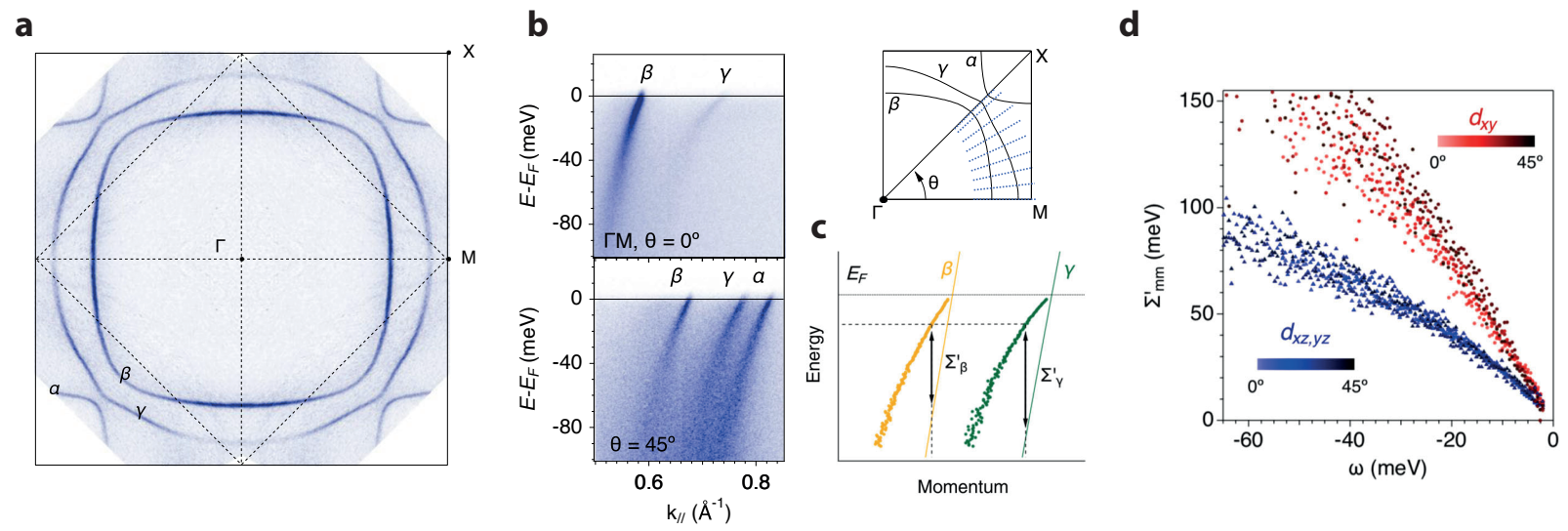

Figure 29: Laser-ARPES measurements of $\mathrm{Sr}_{2} \mathrm{RuO}_{4} \cdot{ }^{274}$ (a) Fermi surface and (b) dispersions measured along high-symmetry directions (see inset). (c) Extracted dispersions for $\theta=0$, and illustration of the procedure to extract the real part of the complex self-energy in the band basis. (d) Corresponding real part of the self energy extracted from several such cuts at different angles around the Brillouin zone, and transformed to the orbital basis as described in Ref. ${ }^{274}$ All panels reproduced with permission from Ref. ${ }^{274}$ under a Creative Commons Attribution 4.0 International License.

utilising a latest-generation $11 \mathrm{eV}$ light source. Profiting from the low photon energy, an excellent momentum resolution is evident, allowing an extremely clear measurement of the Fermi surface of $\mathrm{Sr}_{2} \mathrm{RuO}_{4}$. It is predominantly derived from the three $t_{2 g}$ orbitals of the $\mathrm{Ru} 4 d$ electrons, from the $\mathrm{RuO}_{2}$ plane of this layered perovskite system (see Figure 24(b)). ${ }^{275}$ The $d_{x y}$ orbitals contribute a nearly circular Fermi surface pocket, while the $d_{x z}$ and $d_{y z}$ orbitals lead to quasi-one-dimensional Fermi surface sheets, reflecting their real-space anisotropy. Pronounced spin-orbit coupling leads to substantial orbital mixing, and the opening of hybridisation gaps along the Brillouin zone diagonals (Figure 29a). ${ }^{276}$

Several ARPES studies to date have observed effective mass enhancements for $\mathrm{Sr}_{2} \mathrm{RuO}_{4}$, consistent with the findings of quantum oscillations. ${ }^{269,270,273,274,277-282}$ A particularly detailed study was carried out in Ref. ${ }^{274}$ As evident in Figure 29b, an increased spectral broadening (indicating a reduced lifetime, see Section 2.3) is evident in the measured dispersions with increasing binding energy. This is a characteristic spectral signature of electron-electron interactions, and occurs in tandem with the reduction of the Fermi velocity discussed above. The quasiparticle renormalization, as referenced to the non-interacting band structure, and 
the decrease of the lifetime are encoded in a complex self-energy (Eqn. 5). This self-energy can be extracted from high-resolution measurements of the form shown here, albeit with some calculations of, or assumptions for, the hypothetical non-interacting "bare" band. This procedure is shown for the real part of the self energy in Figure 29c. This analysis procedure thus provides direct insight into the nature and strength of the electronic interactions in the solid. Intriguingly, the self-energy extracted in this way was shown to have a strongly varying magnitude for different cuts throughout the Brillouin zone. ${ }^{274}$ This might naively suggest a strong momentum-dependence to the interaction. Nonetheless, recasting the extracted selfenergy from the band basis to the orbital basis, accounting for the variable orbital mixing around the Fermi surface discussed above, the extracted self energies at all momenta collapse onto single orbital-specific curves (Figure 29d). ${ }^{274}$ This important result indicates that the self energies are in fact local in the orbital basis in $\mathrm{Sr}_{2} \mathrm{RuO}_{4}$, validating, at least for this model system, an assumption of $\mathbf{k}$-independent self energies that is often applied in the analysis of photoemission data.

The multi-band nature of $\mathrm{Sr}_{2} \mathrm{RuO}_{4}$ has further rendered it an attractive candidate for tuning its electronic properties and many-body interactions via chemical and pressure control, with ARPES providing valuable feedback to the efficacy of each method. Of particular interest is a saddle-point in the $\gamma$-band electronic structure, located at the M point of the Brillouin zone. Driving this van Hove singularity, with its large peak in the electronic density of states, to the Fermi level can be expected to have substantial consequences for the many-body interactions and the electronic phases of this system. ${ }^{283}$

As shown in Figure 30a(i), the top of the hole-like dispersion along $\Gamma-\mathrm{M}$, which marks the location of the van Hove singularity, is located above $E_{\mathrm{F}}$. As ARPES is a filled-state spectroscopy, the top of the band is not visible in measurements performed at low temperature. However, it is possible to utilise thermal population of states above the chemical potential. Figure 30a(ii) shows equivalent measurements performed at a temperature of $100 \mathrm{~K}$, with the measured spectra divided by the Fermi-Dirac distribution function for this 


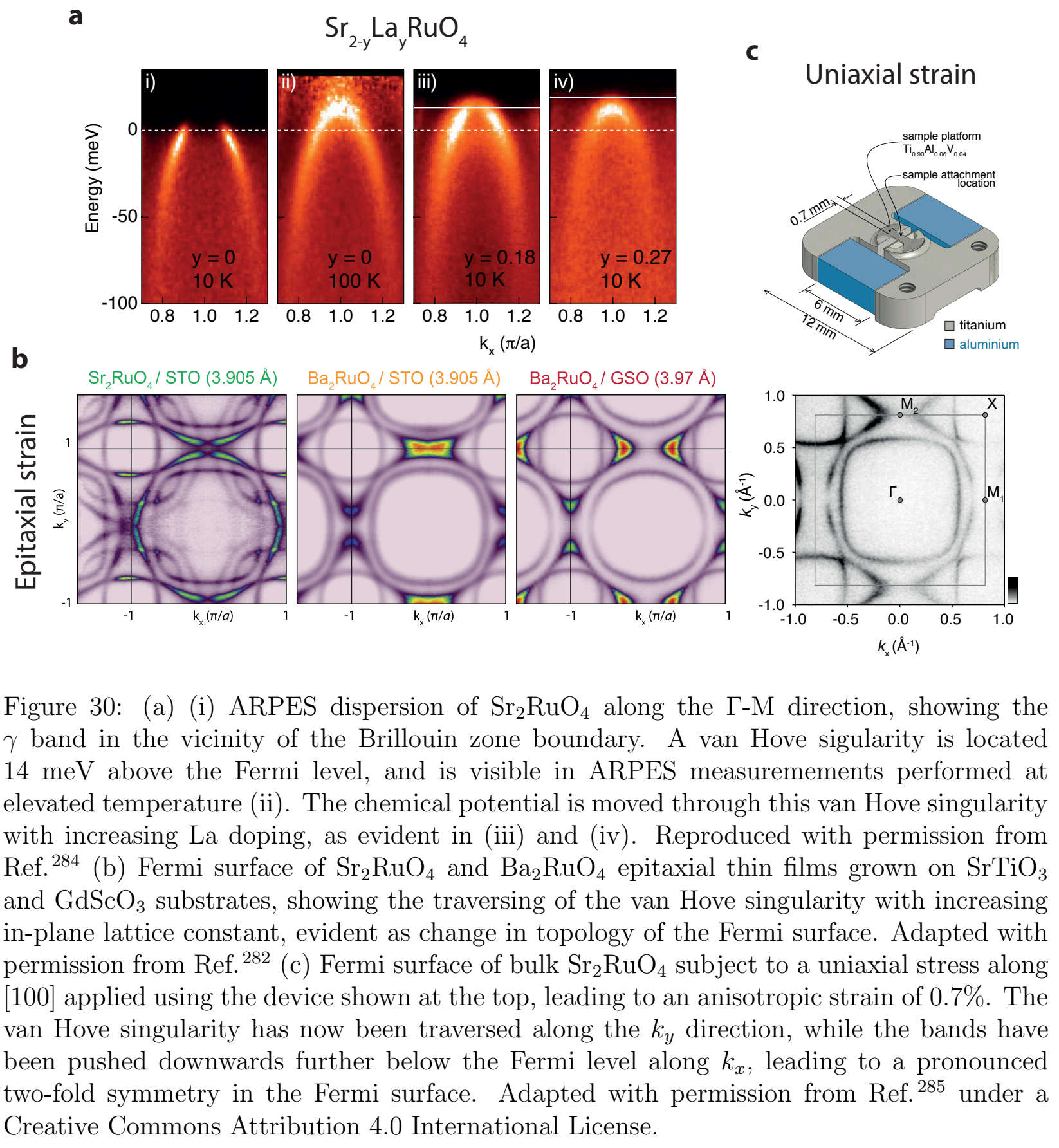

elevated temperature. In this way, it becomes possible to observe the band top along this direction, and via this, to locate the van Hove singularity in $\mathrm{Sr}_{2} \mathrm{RuO}_{4}$ at $14 \mathrm{meV}$ above the Fermi level. ${ }^{284}$ This is close enough to $E_{\mathrm{F}}$ to be accessible via chemical doping, where again ARPES can be used to track the evolution of the position of the van Hove singularity. Figure 30a(iii,iv) shows that a substitution of La for Sr leads to the expected electron doping, 
with detailed analysis showing that this leads to an effective rigid band shift. ${ }^{284}$ A composition of $\mathrm{Sr}_{1.82} \mathrm{La}_{0.18} \mathrm{RuO}_{4}$ brings the van Hove singularity very close to the Fermi level (solid line in Figure 30a(iii), while the van Hove singularity is clearly pushed below the Fermi level for a composition of $\mathrm{Sr}_{1.73} \mathrm{La}_{0.27} \mathrm{RuO}_{4}$, accompanied by a topological change of the $\gamma$ Fermi surface from electron-like to hole-like. ${ }^{284}$

A similar change in the Fermi surface topology can be observed in Figure 30b, where epitaxial strain is employed as the tuning parameter. ${ }^{282}$ For $\mathrm{Sr}_{2} \mathrm{RuO}_{4}$ coherently strained to a $\mathrm{SrTiO}_{3}$ substrate (applying tensile strain), the $\gamma$ Fermi surface sheets are now much closer to touching at the Brillouin zone boundary than for bulk $\mathrm{Sr}_{2} \mathrm{RuO}_{4}$ (Figure 29a), indicating the closer proximity of the van Hove singularity to the Fermi level. With additional chemical pressure from the replacement of $\mathrm{Sr}$ with $\mathrm{Ba}$, the van Hove singularity is moved just below the Fermi level, while additional epitaxial strain induced by a $\mathrm{GdScO}_{3}$ substrate makes the Lifshitz transition clear, with hole-like $\gamma$ Fermi surfaces now located around the Brillouin zone corners. This is a clear indication of the power of thin-film growth approaches for tuning the electronic structure of complex oxides, and of the benefit of combining these with in situ ARPES capabilities to provide direct spectroscopic feedback of the evolution of the electronic structure and quantum many-body interactions. ${ }^{282,286-288}$

As a final method for tuning the electronic structure of $\mathrm{Sr}_{2} \mathrm{RuO}_{4}$, we show in Figure 30c Fermi surface measurements of a sample subject to a uniaxial stress. Achieving large uniaxial strains in single-crystal samples, within the size and vacuum constraints of a typical photoemission experiment, is technically challenging. Nonetheless, several spring- and piezo-based sample rigs have been developed to allow detwinning of multi-domain samples, which have been extensively used to study the electronic structure of Fe-based supreconductors. ${ }^{289-292}$ Recently, several sample rigs have also been developed, based on mechanical actuation or differential thermal contraction of components on the sample plate, in order to apply large and tuneable uniaxial stress to single-crystal samples, in a geometry that leaves optical access to the sample and is suitable for performing high-resolution ARPES experiments. ${ }^{285,293,294}$ One 
such device (Figure 30c) has recently been applied to study the uniaxial strain-evolution of the electronic structure of $\mathrm{Sr}_{2} \mathrm{RuO}_{4} \cdot{ }^{285}$ Uniaxial strain is a particularly clean tuning parameter, and has been shown to lead to a remarkable increase (by more than factor of two) and peak in the superconducting transition temperature of $\mathrm{Sr}_{2} \mathrm{RuO}_{4} \cdot{ }^{283}$ ARPES measurements, performed with a uniaxial strain, $\varepsilon_{x x}-\varepsilon_{y y}$ as high as $-0.7 \%$, allow tracking the change in Fermiology that underpins this. Figure 30c shows that the Fermi surface has again undergone a Lifshitz transition as compared to unstrained $\mathrm{Sr}_{2} \mathrm{RuO}_{4}$, with a van Hove singularity moving below the Fermi level. Now, however, this occurs only for the direction corresponding to induced tensile strain, while the $\gamma$ sheet Fermi surface moves further away from the Brillouin zone boundary for the direction corresponding to applied compressive strain, indicating that the van Hove singularity moves further above the Fermi level for this direction. The Fermi surface thus develops a pronounced two-fold symmetry. Quantitative comparison with strain-driven evolution of the electronic structure shows some pronounced discrepancies with the predictions of first-principles calculations in the vicinity of the Lifshitz transition, indicating a possible changing role of many-body interactions as the van Hove singularity is approached. ${ }^{285}$ Such studies of strain-driven changes in electronic structure are in their infancy, and we would expect more elaborate strain-ARPES approaches to be developed over the coming years. These can be expected to provide powerful insights on how externallyapplied, as well as epitaxially-induced strain, manipulate the many-body states and phases of quantum materials.

\section{$6 \quad$ Future directions and technical challenges}

As already evident from some of the results presented above, recent technological developments have continued to expand the range of applicability of ARPES and the information that can be obtained. Here, we briefly summarise some of these developments and the opportunities and challenges for exploiting them in future. 


\subsection{Micro and Nano-ARPES}

Traditionally, ARPES measurements are performed by illuminating the sample with a spot of typical dimensions from ca. $50 \mu \mathrm{m}$ to ca. $1 \mathrm{~mm}$, depending on the setup. This means that the information obtained reflects a spatial average over relatively large length scales. There has been increased interest in focussing the light spot used for photoemission, which can then be scanned across the sample in order to provide real-space selectivity. The result is a form of spectro-microscopy, where (AR)PES measurements provide a powerful contrast mechanism. This has found increasingly widespread use, from studies of electronically-inhomogeneous phases $^{295}$ through to the investigation of materials with limited spatial dimensions (e.g. exfoliated flakes of transition-metal dichalcogenides - see Figures 19 and 23). Current approaches have reached sub- $\mu \mathrm{m}$ spatial resolution.

The ongoing technical development of synchrotron storage rings, including many planned future upgrades worldwide, have targeted so-called diffraction-limited performances, where the increased brightness due to the reduction of the source dimension, among other specific advantages, will allow optimization of extremely focused radiation. ${ }^{296}$ Although the useful flux for ARPES experiment is intrinsically limited by so-called space charge effects (arising from the Coulomb repulsion between the photoemitted electrons), a small spot will bring significant advantages in effective energy and angular resolution, and one may expect spectromicroscopy will be routinely used to monitor the evolution of size dependent phenomena and nanostructures, as e.g. embedded nanoparticles, photosensitive reactions and catalytic effects, multi and stripe-phase orders.

As a function of the achieved spot at the sample position, different technical solutions have been adopted, leading to micro-ARPES and nano-ARPES spectro-microscopy, ${ }^{297}$ both of them building on specific requirements of optic elements. Looking at the more representative approaches, the field of spectro-microscopy at synchrotron radiation beamlines has been

initiated by the pioneering work of Kirkpatrick and Baez, ${ }^{298}$ where a geometrical tandem arrangement of two toroidal mirrors is used to obtain typically a few micron of focal spot. 
The drawback of this approach when applied to ARPES is the unpractical short distance (in the range of some $\mathrm{cm}$ ) between the optics (and their mechanical supports and kinematics) and the sample.

Submicrometric spot size is also achievable with the use of Schwarzschild objectives, a reflective microscope objective optimized for high reflectivity. ${ }^{299}$ Although well adapted to ARPES setups, such approach suffers the lack of photon energy tuneability. Thanks to the increased brilliance of sources and in perspective of future upgrades of synchrotron radiation facilities, the dimension of the spot at the sample position is expected to scale down to the nm range by the use of special diffractive objectives, the zone plates, in combination with dedicated focusing mirrors and miniaturization efforts. ${ }^{297}$ When highest transmission and a somehow relaxed spatial resolution (on the order of hundreds of nm to a few microns) are needed, a recent complementary approach has been demonstrated by the use of capillary optics, typically based on ellipsoidal coated mirrors. ${ }^{300}$ Important examples of spectro-microscopy setups have not only been developed at synchrotron sources, but also in a laboratory environment, where laser-based Micro and Nano-ARPES instruments have been succesfully developed with high energy and spatial resolution. ${ }^{301-304}$

It is important to underline that non only spectro-microscopy, i.e. the use of a small spot combined with a classic detector (see section 2) but also micro-spectroscopy, consisting in the illumination of the sample with a larger spot and the achievement of micro/nano lateral resolution by means of electrostatic/magnetic optics, has been successfully developed in recent years with outstanding examples in PEEM/LEEM spectroscopy, and more recently with the so-called momentum microscope. We refer the reader to specific publications and review books focused on this argument. ${ }^{305-311}$

The reduced spot is also expected to boost so-called in-operando spectroscopy, including ARPES. In-operando experiments are one of the next frontier of experiments with X-rays since the intermediate steps in synthesis and assembly of nano and quantum materials can in principle be monitored in real time yielding information that will allow designing more 
effective and efficient synthesis routes. So far, this approach has been successfully developed for the so-called ambient pressure photoemission and soft X-ray ARPES. ${ }^{312-314}$ In light of the theoretical difficulties in predicting pathways, this capability is urgently needed for developing efficient nano-fabrication with tailored functionalities. Most systems are synthesized under non-equilibrium conditions, thus performing analytic measurements in complex and "real" conditions, such as in gas-liquids and gas-solid environment will be a major achievement in the field. In particular, for nano-catalysis, a key issue is to develop the ability to monitor nano-particle chemical reactions in real time. ${ }^{315}$ The study of processes at the nanoscale "while they occur" is of core relevance for synthesis and chemical analysis, both at large facilities and in lab-environment. ${ }^{314,316}$ In addition, in-operando studies as a function of electrical control, e.g. electrical gating (Figure 19) are also likely to find increasingly widespread use in the coming years.

\subsection{Time Resolved ARPES}

ARPES is a filled-state spectroscopy, and as such provides access only to the electronic structure below the Fermi level. As discussed in Section 5.2.2, it is possible to transiently occupy the nominally unoccupied states via photoexcitation, and to make use of stroboscopic ARPES measurements from this excited state to track how the resulting excited state carrier dynamics evolve. This is typically achieved using ultrafast laser-based setups. A pump pulse is used to photo-excite the system, while a separate probe pulse, separated by a well-defined and variable time delay, is used to induce photoemission. The probe pulse must therefore be of sufficiently high photon energy, and is typically generated by either sequential stages of frequency doubling or sum-frequency generation of pulsed laser sources using non-linear optical crystals, or via high-harmonic generation (HHG) in the gas phase. ${ }^{303}$ The former are typically limited to a single lower energy, but can often be achieved with more compact

setups, and with higher repetition rates and higher energy resolutions. ${ }^{303}$ The later give access to higher photon energies (and thus access to a larger portion of the Brillouin zone), 
which are often tuneable. The pulse length is normally on the order of a few tens or hundreds of fs, allowing access to ultrafast carrier dynamics. The time-bandwidth product, however, means that the shorter the pulse duration, the worse the achievable energy resolution. A choice must therefore be made between required temporal and energy resolution. Energetic shifts and broadening of the measured spectra due to space-charge effects can be particularly problematic for time-resolved ARPES, especially for instruments where the repetition rate is low and thus a high photon number per pulse is required. ${ }^{317}$ This has proved a major constraint for realising ARPES studies using free electron lasers (FELs), ${ }^{318}$ which typically generate ultrashort ultra-intense pulses with low repetition-rate. However, the first time and momentum-resolved ARPES studies utilising FELs have now been reported, ${ }^{306}$ and this is likely to become increasingly utilised as high-repetition-rate FELs become more available. Meanwhile, recent advances in the generation of XUV photons from HHG sources, and the more wide-spread availability of these, is currently strongly increasing the impact and applicability of TR-ARPES experiments in the laboratory setting. ${ }^{304,319-324}$ Optical pumpprobe setups are also being combined with efficient electron detection schemes such as timeof-flight analysers ${ }^{324}$ and momentum-microscopes, ${ }^{307}$ giving access to an impressive amount of information on non-equilibrium states in solids.

In general, accessing the time-domain parameter space in ARPES experiments opens up a wide range of avenues for controlling and imaging the electronic structures of solids. Beyond photodoping to occupy conduction band states, it allows the tracking and tuning of many-body interactions, ${ }^{325,326}$ driving phase transitions, ${ }^{327,328}$ establishing the characteristic timescales for the melting of electronic orders, ${ }^{329,330}$ creating and imaging photon-dressed states, ${ }^{331}$ and more. A full treatment of this exciting area is beyond the scope of this review, and we refer the interested reader to several review articles that are specifically focused on laser-based and time-resolved photoemission. ${ }^{303,332}$ We note here, however, that given the breadth and complexity of the systems and phenomena that can be studied in this way, time-resolved ARPES is likely to receive significant and increasing utilisation in the coming 
years.

\subsection{Integrating growth and ARPES}

One of the future directions of the next decade is to develop the ability to create 'on-demand' quantum materials. ${ }^{227}$ Both single-layer controllability and design of hybrid hetero-structures are needed, and quantum material synthesis is rapidly progressing in this direction. ${ }^{10,333,334}$ Controlled defect-chemistry and controlled doping, i.e. defying the solubility limit, are also expected to play an important role, following results and achievements obtained with metal oxides, ${ }^{335}$ and more recently in molecular graphene nanostructures. ${ }^{336}$ ARPES will have a critical role in the development of 'ad-hoc' synthesis of quantum materials, exploiting on the one hand its direct access to $k$-space and electronic structure, and on the other hand the comparison with theoretical prediction on novel interfacial electronic phases and states of matter which it facilitates. ${ }^{286}$ This, however, requires a full integration of growth (e.g. molecular beam epitaxy or pulsed layer deposition) and characterisation, in large and complex experimental setups. Several systems to achieve this have now been developed both

in university labs and integrated with synchrotron beamlines at large scale facilities ${ }^{36,337-339}$ (see e.g. Figures 18, 20, and 30 of this review for some recent results obtained exploiting such capabilities). The development of common protocols in controlled environment (e.g. in-situ transfer) will be beneficial to further the increase of reliability and repeatability of such experiments.

\subsection{The future of spin-resolved ARPES}

As outlined in previous sections, the capability of disentangling spin-related information in quantum materials has been the 'fil-rouge' of technological developments and scientific outcomes; since the breakthrough experimental determination of spin-momentum locking in the first family of topological insulators such as $B i_{2} S e_{3}$ and $B i_{2} T e_{3}$, the interplay between spin, topology and magnetism in many relevant systems has reinforced the need for efficient 
and reliable spin detectors. ${ }^{52-55,58}$ New effects and new quantum quasiparticles have been recently predicted and observed. These include amongst many others axion insulators, Chern insulators, single and few-layers van der Waals ferromagnets, and layered antiferromagnetic insulators. In all these cases, knowledge of spin properties (both in magnetic and nonmagnetic environments) is a fundamental prerequisite for technological applications. We believe that spin resolved ARPES will continue to play a relevant role in this field and we anticipate that multi channel spin filter apparatus will be developed further with respect the present state0of-the-art, reaching higher efficiency by means of parallel acquisition. ${ }^{59}$ Multidimensional ARPES, where for example spin, momentum and time resolution will be jointly at hand is certainly one major axis of development in ARPES-based science.

\section{Conclusions and Perspectives}

One of the next frontiers in research on the quantum properties of solids will be developing the ability of understanding, designing, and controlling, i.e. 'building' systems with tailored structural, electronic and magnetic properties, directly connected with a whole new world of emergent new collective phenomena. To name but a few, self-assembly and self-ordering, quantum size confinement, wave-like transport, orbitally/magnetically driven effects, spinorbit coupled phases, non-trivial topologies, and strong electronic correlations must all be understood, integrated, and their interplay controlled. The study of condensed matter systems exhibiting emergent effects not only in their bulk, but also in thin layers and via interfacial phenomena provides a promising research field with a wide potential and multi-disciplinary research objectives.

Angle-resolved photoelectron spectroscopy provides invaluable insights into the ordered states of quantum materials, by directly probing how their electrons - at the heart of their complex phases - move and interact in the solid. We have presented a few recent examples focused on topological insulators/spin-orbit coupled states, interface and bulk 2-dimensional 
metallic phases in transition-metal oxides, and orbitally/spin driven effects in transition metal dichalcogenides. The range of applicability of the technique is, however, much broader, from studying halide perovskites used in solar cells ${ }^{340}$ to probing the fundamental physics of high-temperature superconductors, ${ }^{268}$ and from studying the surface physics and chemistry of nanowires ${ }^{328}$ to inducing and probing new hybrid light-matter states. ${ }^{331}$ Angle-resolved photoelectron spectroscopy can thus be expected to play an important role in the study of the electronic properties of solids across a broad array of materials systems and phases. Indeed, quantum materials are characterised by their complex chemistry, physics, and the link between these. In order to reach a step forward in the control of their functionalities, a fundamental understanding of the link between synthesis processes and functional behaviour must be gained. To address such a goal, advanced synthesis and growth capabilities must be developed in combination with analytical tools and spectroscopies, and we consider that angle-resolved photoelectron spectroscopy has an important role to play in that area.

\section{Biographies}

Phil King is Professor of Physics at the University of St Andrews, UK. He received his MPhys and PhD degrees from the Universities of Oxford and Warwick, UK, respectively, before undertaking postdoctoral research in St Andrews and Cornell. Since 2013 he has been on the faculty at St Andrews, where his research has focussed on the application of angle-resolved photoemission spectroscopy to the study of quantum materials, in particular transition-metal oxides and dichalcogenides.

Silvia Picozzi received her BSc in Physics at Univ. L'Aquila, Italy (1994) and her PhD in Materials Science from Univ. Camerino, Italy (1998). She is Director of Research at Consiglio Nazionale delle Ricerche (CNR), Institute for Superconducting and Innovative materials and Devices (SPIN) in Chieti (Italy). Her activity is related to materials modeling (mostly simulations based on density functional theory, but also symmetry analysis 
and model Hamiltonian) in the field of functional materials (such as ferroelectrics, ferromagnets, multiferroics) and materials with strong spin-orbit interaction (i.e. Rashba-Dresselhaus effects, topological matter).

Russell G. Egdell Russell G. Egdell is Emeritus Professor of Inorganic Chemistry in the University of Oxford and Emeritus Fellow of Trinity College, Oxford. He has worked on both gas phase and solid state photoelectron spectroscopy for over 45 years. His recent

research is at the borderline between solid state chemistry and solid state physics, with a focus on the use of X-ray spectroscopies to probe the electronic properties of metal oxides and their surfaces.

Giancarlo Panaccione is Director of Research at the Consiglio Nazionale delle Ricerche (CNR), IOM Institute in Trieste (Italy). He received in 1995 his $\mathrm{PhD}$ in Material Science at the University Paris-VI (France). He moved from France to Italy on 1998, hired by Istituto Nazionale Fisica per la Materia (INFM). His activity is mostly devoted to the exploitation of X-ray based electron spectroscopies (including Synchrotron Radiation) for the study of correlated systems and novel quantum materials, following three main axes: electronic and magnetic properties of low dimensional systems (surfaces and interfaces), electron confinement and complex oxides.

\section{Acknowledgement}

This work is dedicated to Charles S. (Chuck) Fadley, who passed away on August 1 ${ }^{\text {st }}, 2019$. The authors gratefully acknowledge valuable discussions with numerous collaborators and group members with whom they have had the privilege to work. GP acknowledges valuable discussions with Ralph Claessen, Tien-Lin Lee and Annabella Selloni, and the support by the scientific and the technical staff of APE-NFFA beamlines and SPRINT-NFFA laboratory. This work has been partly performed in the framework of the Progetto Internazionale Nanoscience Foundry and Fine Analysis (NFFA-MIUR) facility. PK gratefully acknowledges 
The Royal Society for support. SP acknowledges support from the Italian Ministry of University and Research (MIUR) under the PRIN-2017 project No. 2017YCTB59 (TWEET: ToWards fErroElectricity in Two-dimensions).

\section{References}

(1) Geim, A. K.; Grigorieva, I. V. Van der Waals Heterostructures. Nature 2013, 499, 419-425.

(2) Ajayan, P.; Kim, P.; Banerjee, K. Two-dimensional van der Waals Materials. Phys. Today 2016, 69, 38-44.

(3) Cao, Y.; Fatemi, V.; Fang, S.; Watanabe, K.; Taniguchi, T.; Kaxiras, E.; JarilloHerrero, P. Unconventional Superconductivity in Magic-Angle Graphene Superlattices. Nature 2018, 556, 43-50.

(4) Cao, Y.; Fatemi, V.; Demir, A.; Fang, S.; Tomarken, S. L.; Luo, J. Y.; SanchezYamagishi, J. D.; Watanabe, K.; Taniguchi, T.; Kaxiras, E.; Ashoori, R. C.; JarilloHerrero, P. Correlated Insulator Behaviour at Half-Filling in Magic-Angle Graphene Superlattices. Nature 2018, 556, 80-84.

(5) Hasan, M. Z.; Kane, C. L. Colloquium: Topological Insulators. Rev. Mod. Phys. 2010, 82, 3045-3067.

(6) Manchon, A.; Koo, H. C.; Nitta, J.; Frolov, S. M.; Duine, R. A. New Perspectives for Rashba Spin-Orbit Coupling. Nat. Mater. 2015, 14, 871-882.

(7) Kim, B. J.; Jin, H.; Moon, S. J.; Kim, J.-Y.; Park, B.-G.; Leem, C. S.; Yu, J.; Noh, T. W.; Kim, C.; Oh, S.-J.; Park, J.-H.; Durairaj, V.; Cao, G.; Rotenberg, E. Novel Jeff=1/2 Mott State Induced by Relativistic Spin-Orbit Coupling in $\mathrm{Sr}_{2} \mathrm{IrO}_{4}$. Phys. Rev. Lett. 2008, 101, 076402. 
(8) Kim, B. J.; Ohsumi, H.; Komesu, T.; Sakai, S.; Morita, T.; Takagi, H.; Arima, T. Phase-Sensitive Observation of a Spin-Orbital Mott State in $\mathrm{Sr}_{2} \mathrm{IrO}_{4}$. Science 2009, 323, 1329-1332.

(9) Mannhart, J.; Schlom, D. G. Oxide Interfaces : An Opportunity for Electronics. Science 2010, 327, 1607-1611.

(10) Hwang, H. Y.; Iwasa, Y.; Kawasaki, M.; Keimer, B.; Nagaosa, N.; Tokura, Y. Emergent Phenomena at Oxide Interfaces. Nat. Mater. 2012, 11, 103-113.

(11) Fadley, C. S. Atomic-level Characterization of Materials with Core- and Valence-Level Photoemission: Basic Phenomena and Future Directions. Surf. Interface Anal. 2008, 40, 1579-1605.

(12) Fadley, C. In in Electron Spectroscopy: Theory, Techniques, and Applications, vol. II, Chap. 1; Brundle, R., Baker, A., Eds.; Academic Press: London, 1978; Vol. II.

(13) Hüfner, S. in Photoelectron Spectroscopy: Principles and Applications (3rd ed), Springer Series in Solid-State Sciences; Springer, Berlin: Berlin, Heidelberg, 2003; Vol. 82.

(14) Damascelli, A.; Hussain, Z.; Shen, Z. X. Angle-resolved Photoemission Studies of the Cuprate Superconductors. Rev. Mod. Phys. 2003, 75, 473-541.

(15) Damascelli, A. Probing the Electronic Structure of Complex Systems by ARPES. Phys. Scripta T 2004, T109, 61-74.

(16) Hüfner, S.; Schmidt, S.; Reinert, F. Photoelectron Spectroscopy - An Overview. Nucl. Instrum. Methods Phys. Res., A 2005, 547, 8-23.

(17) Grioni, M. In A Photoemission Primer, in Magnetism and Synchrotron Radiation. Lecture Notes in Physics; Beaurepaire, E., Kappler, J. P., Scheurer, F., Eds.; Springer, Berlin, Heidelberg: Berlin, Heidelberg, 2001; Vol. 565; pp 109-128. 
(18) Sing, M.; Claessen, R. In Photoelectron Spectroscopy of Transition-Metal Oxide Interfaces, in Spectroscopy of Complex Oxide Interfaces; Cancellieri, C., Strocov, V., Eds.; Springer International Publishing: Boston (MA), 2018; Vol. 266.

(19) Klein, A.; Mayer, T.; Thissen, A.; Jaegermann, W. Photoelectron Spectroscopy in Materials Science and Physical Chemistry :. Bunsen- Magazin 2008, 124-139.

(20) The Nobel Nomination Archive, https://www.nobelprize.org/nomination/archive/.

(21) Robinson, H. R.; Young, C. L. The Influence of Chemical State on Critical X-ray Absorption Frequencies. The London, Edinburgh and Dublin Philosophical Magazine and Journal of Science 1930, 10, 71-75.

(22) Siegbahn, K.; Nordling, C.; ; Johansson, C.; Hedman, J.; P.-F., H.; Hamrin, K.; Gelius, U.; Bergmark, T.; Werme, L.; Manne, Y.; Baer, Y. ESCA Applied to Free Molecules,; North-Holland: Amsterdam, The Netherlands,, 1969.

(23) Siegbahn, K.; Nordling, C.; Fahlman, A.; Hamrin, K.; Hedman, J.; Nordberg, R.; Johansson, C.; Bergmark, T.; Karlsson, S. E.; Lindgren, I.; B. Lindberg, B. Atomic, Molecular and Solid- state Structure Studied by Means of Electron Spectroscopy. Nova Acta Regiae Soc. Sci. Ups. 1967, 201-282.

(24) Einstein, A. Concerning an Heuristic Point of View Toward the Emission and Transformation of Light. Annalen der Physik 1905, 17, 132-148.

(25) In fact, $\phi$ is the work function of the spectrometer used to measure the kinetic energy, as the spectrometer is in direct electrical contact with the solid sample.

(26) Gedik, N.; Vishik, I. Photoemission of Quantum Materials. Nat. Phys. 2017, 13, 1029-1033.

(27) Walsh, A.; Sokol, A. A.; Buckeridge, J.; Scanlon, D. O.; Catlow, C. R. A. Oxidation States and Ionicity. Nat. Mater. 2018, 17, 958-964. 
(28) Gray, A. X.; Papp, C.; Ueda, S.; Balke, B.; Yamashita, Y.; Plucinski, L.; Minár, J.; Braun, J.; Ylvisaker, E. R.; Schneider, C. M.; Pickett, W. E.; Ebert, H.; Kobayashi, K.; Fadley, C. S. Probing Bulk Electronic Structure with Hard X-ray Angle-Resolved Photoemission. Nat. Mater. 2011, 10, 759-764.

(29) Ishida, Y.; Shin, S. Functions to map Photoelectron Distributions in a Variety of Setups in Angle-Resolved Photoemission Spectroscopy. Rev. Sci. Instrum. 2018, 89, 043903.

(30) Hansson, G. V.; Uhrberg, R. I. G.; Flodström, S. A. Electronic Structure of the Si(111)7x7 Surface studied by Angle-Resolved Photoelectron Spectroscopy. J. Vac. Sci. Technol. 1979, 16, 1287-1289.

(31) Sunko, V. et al. Maximal Rashba-like Spin Splitting via Kinetic-energy-coupled Inversion-Symmetry Breaking. Nature 2017, 549, 492-496.

(32) Aebi, P.; Fasel, R.; Naumović, D.; Hayoz, J.; Pillo, T.; Bovet, M.; Agostino, R. G.; Patthey, L.; Schlapbach, L.; Gil, F. P.; Berger, H.; Kreutz, T. J.; Osterwalder, J. Angle-scanned Photoemission: Fermi Surface Mapping and Structural Determination. Surf. Sci. 1998, 402-404, 614-622.

(33) Aebi, P.; Osterwalder, J.; Fasel, R.; Naumović, D.; Schlapbach, L. Fermi Surface Mapping with Photoelectrons at UV Energies. Surf. Sci. 1994, 307-309, 917-921.

(34) Rotenberg, E.; Theis, W.; Horn, K.; Gille, P. Quasicrystalline Valence Bands in Decagonal AlNiCo. Nature 2000, 406, 602-605.

(35) Lindroos, M.; Bansil, A. A novel Direct Method of Fermi Surface Determination using constant initial Energy Angle-scanned Photoemission Spectroscopy. Phys. Rev. Lett. 1996, 77, 2985-2988. 
(36) Hoesch, M. et al. A Facility for the Analysis of the Electronic Structures of Solids and their Surfaces by Synchrotron Radiation Photoelectron Spectroscopy. Rev. Sci. Instrum. 2017, 88, 013106.

(37) Baumberger, F.; Ingle, N. J. C.; Meevasana, W.; Shen, K. M.; Lu, D. H.; Perry, R. S.; Mackenzie, A. P.; Hussain, Z.; Singh, D. J.; Shen, Z.-X. Fermi Surface and Quasiparticle Excitations of $\mathrm{Sr}_{2} \mathrm{RhO}_{4}$. Phys. Rev. Lett. 2006, 96, 246402.

(38) Lazzeri, M.; Selloni, A. Stress-driven Reconstruction of an Oxide Surface: the Anatase $\mathrm{TiO}_{2}(001)-(1 \times 4)$ Surface. Phys. Rev. Lett. 2001, 87, 266105.

(39) Bigi, C. et al. Distinct Behavior of Localized and Delocalized Carriers in Anatase $\mathrm{TiO}_{2}(001)$ during reaction with $\mathrm{O}_{2}$. Phys. Rev. Mater. 2020, 4, 025801.

(40) Wang, Z.; Zhong, Z.; McKeown Walker, S.; Ristic, Z.; Ma, J.-Z.; Bruno, F. Y.; Riccò, S.; Sangiovanni, G.; Eres, G.; Plumb, N. C., et al. Atomically precise lateral modulation of a two-dimensional electron liquid in anatase $\mathrm{TiO}_{2}$ thin films. Nano Lett. 2017, 17, 2561-2567.

(41) Gobaut, B.; Orgiani, P.; Sambri, A.; di Gennaro, E.; Aruta, C.; Borgatti, F.; Lollobrigida, V.; Céolin, D.; Rueff, J.-P.; Ciancio, R., et al. Role of Oxygen Deposition Pressure in the formation of Ti Defect States in $\mathrm{TiO}_{2}(001)$ Anatase Thin Films. ACS Appl. Mater. E3 Inter. 2017, 9, 23099-23106.

(42) González, M. S. M.; Aguirre, M. H.; Morán, E.; Alario-Franco, M. Á.; Perez-Dieste, V.; Avila, J.; Asensio, M. C. In-situ Reduction of (100) $\mathrm{SrTiO}_{3}$. Solid State Sciences 2000, 2, 519-524.

(43) De Leon-Guevara, A.; Berthet, P.; Berthon, J.; Millot, F.; Revcolevschi, A.; Anane, A.; Dupas, C.; Le Dang, K.; Renard, J.; Veillet, P. Influence of Controlled Oxygen Vacancies on the Magnetotransport and Magnetostructural Phenomena in $L a_{0.85} S_{0.15} \mathrm{MnO}_{3-\delta}$ Single Crystals. Phys. Rev. B 1997, 56, 6031-6035. 
(44) Rödel, T.; Vivek, M.; Fortuna, F.; Le Fèvre, P.; Bertran, F.; Weht, R.; Goniakowski, J.; Gabay, M.; Santander-Syro, A. Two-dimensional Electron Systems in a $\mathrm{ATiO}_{3}$ Perovskites $(\mathrm{A}=\mathrm{Ca}, \mathrm{Ba}, \mathrm{Sr})$ : Control of Orbital Hybridization and Energy Order. Phys. Rev. B 2017, 96, 041121.

(45) Moser, S.; Moreschini, L.; Jaćimović, J.; Barišić, O.; Berger, H.; Magrez, A.; Chang, Y.; Kim, K.; Bostwick, A.; Rotenberg, E., et al. Tunable Polaronic Conduction in Anatase $\mathrm{TiO}_{2}$. Phys. Rev. Lett. 2013, 110, 196403.

(46) Plumb, N. C.; Kobayashi, M.; Salluzzo, M.; Razzoli, E.; Matt, C. E.; Strocov, V. N.; Zhou, K. J.; Shi, M.; Mesot, J.; Schmitt, T.; Patthey, L.; Radović, M. Evolution of the $\mathrm{SrTiO}_{3}$ Surface Electronic State as a function of $\mathrm{LaAlO}_{3}$ Overlayer Thickness. Appl. Surf. Sci. 2017, 412, 271-278.

(47) Santander-Syro, A.; Copie, O.; Kondo, T.; Fortuna, F.; Pailhes, S.; Weht, R.; Qiu, X.; Bertran, F.; Nicolaou, A.; Taleb-Ibrahimi, A., et al. Two-Dimensional Electron Gas with Universal Subbands at the Surface of $\mathrm{SrTiO}_{3}$. Nature 2011, 469, 189.

(48) King, P. D. C.; Walker, S. M.; Tamai, A.; De La Torre, A.; Eknapakul, T.; Buaphet, P.; Mo, S.-K.; Meevasana, W.; Bahramy, M.; Baumberger, F. Quasiparticle Dynamics and Spin-Orbital Texture of the $\mathrm{SrTiO}_{3}$ Two-Dimensional Electron Gas. Nat. Commun. 2014, 5, 3414 .

(49) Hsieh, D. et al. A Tunable Topological Insulator in the Spin Helical Dirac Transport Regime. Nature 2009, 460, 1101-1105.

(50) Zhu, Z. H.; Veenstra, C. N.; Levy, G.; Ubaldini, A.; Syers, P.; Butch, N. P.; Paglione, J.; Haverkort, M. W.; Elfimov, I. S.; Damascelli, A. Layer-by-layer Entangled Spin-Orbital Texture of the Topological Surface State in $B i_{2} S e_{3}$. Phys. Rev. Lett. 2013, 110, 1-5.

(51) Kessler, J. Polarized Electrons, 1st ed.; Springer-Verlag Berlin Heidelberg: Chichester, United Kingdom, 1976. 
(52) Hillebrecht, F. U.; Jungblut, R. M.; Wiebusch, L.; Roth, C.; Rose, H. B.; Knabben, D.; Bethke, C.; Weber, N. B.; Manderla, S.; Rosowski, U.; Kisker, E. High-efficiency Spin Polarimetry by Very-low-energy Electron Scattering from Fe(100) for Spin-Resolved Photoemission. Rev. Sci. Instrum. 2002, 73, 1229-1234.

(53) Hoesch, M.; Greber, T.; Petrov, V. N.; Muntwiler, M.; Hengsberger, M.; Auwärter, W.; Osterwalder, J. Spin-polarized Fermi Surface Mapping. J. Electron Spectros. Relat. Phenomena 2002, 124, 263-279.

(54) Bigi, C.; Das, P. K.; Benedetti, D.; Salvador, F.; Krizmancic, D.; Sergo, R.; Martin, A.; Panaccione, G.; Rossi, G.; Fujii, J.; Vobornik, I. Very Efficient Spin Polarization Analysis (VESPA): New Exchange Scattering-based Setup for Spin-resolved ARPES at APE-NFFA Beamline at Elettra. J. Synchrotron Radiat. 2017, 24, 750-756.

(55) Okuda, T.; Takeichi, Y.; Maeda, Y.; Harasawa, A.; Matsuda, I.; Kinoshita, T.; Kakizaki, A. A New Spin-polarized Photoemission Spectrometer with very high Efficiency and Energy Resolution. Rev. Sci. Instrum. 2008, 79, 123117.

(56) Bertacco, R.; Onofrio, D.; Ciccacci, F. A novel Electron Spin-Polarization Detector with very large Analyzing Power. Rev. Sci. Instrum. 1999, 70, 3572-3576.

(57) Okuda, T.; Miyamaoto, K.; Miyahara, H.; Kuroda, K.; Kimura, A.; Namatame, H.; Taniguchi, M. Efficient Spin-resolved Spectroscopy Observation Machine at Hiroshima Synchrotron Radiation Center. Rev. Sci. Instrum. 2011, 82, 103302.

(58) Winkelmann, A.; Hartung, D.; Engelhard, H.; Chiang, C. T.; Kirschner, J. High Efficiency Electron Spin Polarization Analyzer based on Exchange Scattering at Fe/W(001). Rev. Sci. Instrum. 2008, 79, 083303.

(59) Medjanik, K. et al. Direct 3D Mapping of the Fermi Surface and Fermi Velocity. Nat. Mater. 2017, 16, 615-621. 
(60) Riley, J. M. et al. Direct Observation of Spin-Polarized Bulk Bands in an InversionSymmetric Semiconductor. Nat. Phys. 2014, 10, 835-839.

(61) Hohenberg, P.; Kohn, W. Inhomogeneous Electron Gas. Phys. Rev. 1964, 136, B864B871.

(62) Kohn, W.; Sham, L. J. Self-Consistent Equations Including Exchange and Correlation Effects. Phys. Rev. 1965, 140, A1133-A1138.

(63) Burke, K.; Wagner, L. O. DFT in a nutshell. International Journal of Quantum Chemistry 2013, 113, 96-101.

(64) Jones, R. O.; Gunnarsson, O. The Density Functional Formalism, its Applications and Prospects. Rev. Mod. Phys. 1989, 61, 689-746.

(65) Jones, R. O. Density Functional Theory: Its Origins, Rise to Prominence, and Future. Rev. Mod. Phys. 2015, 87, 897-923.

(66) Day, R. P.; Zwartsenberg, B.; Elfimov, I. S.; Damascelli, A. Computational Framework Chinook for Angle-Resolved Photoemission Spectroscopy. npj Quantum Mater. 2019, 4,54 .

(67) Moser, S. An Experimentalist's Guide to the Matrix Element in Angle Resolved Photoemission. J. Electron Spectros. Relat. Phenomena 2017, 214, 29-52.

(68) Das, P. K. et al. Role of Spin-Orbit Coupling in the Electronic Structure of $\operatorname{IrO}_{2}$. Phys. Rev. Mater. 2018, 2, 065001.

(69) Henk, J.; Schattke, W. A Subroutine Package for Computing Green's Functions of Relaxed Surfaces by the Renormalization Method. Computer Physics Commun. 1993, $77,69-83$. 
(70) Cerdá, J.; Van Hove, M.; Sautet, P. Efficient Method for the Simulation of STM Images. I. Generalized Green-function Formalism. Phys. Rev. B 1997, 56, 1588515899.

(71) Woicik, J., Ed. Hard X-ray Photoelectron Spectroscopy (HAXPES), Springer Series in Surface Sciences; Springer International Publishing: Switzerland, 2016; Vol. 59.

(72) Takegami, D.; Nicolai,, L.; Koethe, T. C.; Kasinathan, D.; Kuo, C. Y.; Liao, Y. F.; Tsuei, K. D.; Panaccione, G.; Offi, F.; Monaco, G.; Brookes, N. B.; Minár, J.; Tjeng, L. H. Valence Band Hard X-ray Photoelectron Spectroscopy on 3d TransitionMetal Oxides containing Rare-Earth Elements. Phys. Rev. B 2019, 99, 165101.

(73) Mo, S. K. et al. Prominent Quasiparticle Peak in the Photoemission Spectrum of the Metallic Phase of $\mathrm{V}_{2} \mathrm{O}_{3}$. Phys. Rev. Lett. 2003, 90, 186403.

(74) Sing, M.; Glawion, S.; Schlachter, M.; Scholz, M. R.; Goß, K.; Heidler, J.; Berner, G.; Claessen, R. Photoemission of a Doped Mott Insulator: Spectral Weight Transfer and a Qualitative Mott-Hubbard Description. Phys. Rev. Lett. 2011, 106, 056403.

(75) Schütz, P.; Di Sante, D.; Dudy, L.; Gabel, J.; Stübinger, M.; Kamp, M.; Huang, Y.; Capone, M.; Husanu, M. A.; Strocov, V. N.; Sangiovanni, G.; Sing, M.; Claessen, R. Dimensionality-Driven Metal-Insulator Transition in Spin-Orbit-Coupled $\mathrm{Sr} I r \mathrm{O}_{3}$. Phys. Rev. Lett. 2017, 119, 256404.

(76) Panaccione, G.; Kobayashi, K. Hard X-ray photoemission Spectroscopy: Variable Depth Analysis of Bulk, Surface and Interface Electronic Properties. Surf. Sci. 2012, $606,125-129$.

(77) Tanuma, S.; Powell, C. J.; Penn, D. R. Calculation of Electron Inelastic Mean Free Paths (IMFPs) VII. Reliability of the TPP-2M IMFP predictive Equation. Surf. Interface Anal. 2003, 35, 268-275. 
(78) Powell, C. J.; Jablonski, A. Surface Sensitivity of X-ray Photoelectron Spectroscopy. Nucl. Instrum. Methods Phys. Res., A 2009, 601, 54-65.

(79) Powell, C.; Tanuma, S. In Inelastic Mean Free Paths, Mean Escape Depths, Information Depths, and Effective Attenuation Lengths for Hard X-ray Photoelectron Spectroscopy in Hard X-ray Photoelectron Spectroscopy (HAXPES), Springer Series in Surface Sciences; Woicik, J., Ed.; Springer International Publishing: Switzerland, 2016; Vol. 59.

(80) Kiss, T.; Kanetaka, F.; Yokoya, T.; Shimojima, T.; Kanai, K.; Shin, S.; Onuki, Y.; Togashi, T.; Zhang, C.; Chen, C. T.; Watanabe, S. Photoemission Spectroscopic Evidence of Gap Anisotropy in an f-electron Superconductor. Phys. Rev. Lett. 2005, 94, 057001.

(81) Eguchi, R. et al. Bulk and Surface Sensitive High-resolution Photoemission Study of Mott-Hubbard Systems $\mathrm{SrVO}_{3}$ and $\mathrm{CaVO}_{3}$. Physica B: Condensed Matter 2006, 378-380, 330-331.

(82) Offi, F.; Iacobucci, S.; Vilmercati, P.; Rizzo, A.; Goldoni, A.; Sacchi, M.; Panaccione, G. Attenuation Lengths of Low-Energy Electrons in Solids: The case of CoO. Phys. Rev. B 2008, 77, 201101.

(83) Borgatti, F. et al. Revisiting the Origin of Satellites in Core-Level Photoemission of Transparent Conducting Oxides: The Case of n-doped $\mathrm{SnO}_{2}$. Phys. Rev. B 2018, 97, 155102.

(84) Lindau, I.; Pianetta, P.; Doniach, S.; Spicer, W. E. X-ray Photoemission Spectroscopy. Nature 1974, 250, 214-215.

(85) Sacchi, M. et al. Quantifying the Effective Attenuation Length in High-Energy Photoemission Experiments. Phys. Rev. B 2005, 71, 155117. 
(86) Scanlon, D. O.; Kehoe, A. B.; Watson, G. W.; Jones, M. O.; David, W. I.; Payne, D. J.; Egdell, R. G.; Edwards, P. P.; Walsh, A. Nature of the Band Gap and Origin of the Conductivity of $\mathrm{PbO}_{2}$ revealed by Theory and Experiment. Phys. Rev. Lett. 2011, $107,246402$.

(87) Payne, D. J.; Egdell, R. G.; Paolicelli, G.; Offi, F.; Panaccione, G.; Lacovig, P.; Monaco, G.; Vanko, G.; Walsh, A.; Watson, G. W.; Guo, J.; Beamson, G.; Glans, P. A.; Learmonth, T.; Smith, K. E. Nature of Electronic States at the Fermi Level of Metallic $\beta-\mathrm{PbO}_{2}$ revealed by Hard X-ray Photoemission Spectroscopy. Phys. Rev. B 2007, 75, 153102 .

(88) Trzhaskovskaya, M. B.; Nefedov, V. I.; Yarzhemsky, V. G. Photoelectron Angular Distribution Parameters for Elements $\mathrm{Z}=1$ to $\mathrm{Z}=54$ in the Photoelectron Energy Range 100-5000 eV. At. Data Nucl. Data Tables 2001, 7r, 97-159.

(89) Trzhaskovskaya, M. B.; Nefedov, V. I.; Yarzhemsky, V. G. Photoelectron Angular Distribution Parameters for Elements $\mathrm{Z}=55$ to $\mathrm{Z}=100$ in the Photoelectron Energy Range 100-5000 eV. At. Data Nucl. Data Tables 2002, 82, 257-311.

(90) Yarzhemsky, V. G.; Nefedov, V. I.; Trzhaskovskaya, M. B.; Band, I. M.; Szargan, R. The Influence of Core Hole Relaxation on the main-line Intensities in X-ray Photoelectron Spectra. J. Electron Spectros. Relat. Phenomena 2002, 123, 1-10.

(91) Payne, D. J.; Paolicelli, G.; Offi, F.; Panaccione, G.; Lacovig, P.; Beamson, G.; Fondacaro, A.; Monaco, G.; Vanko, G.; Egdell, R. G. A Study of Core and Valence Levels in $\beta-\mathrm{PbO}_{2}$ by Hard X-ray Photoemission. J. Electron Spectros. Relat. Phenomena 2009, 169, 26-34.

(92) Wang, W.; Deng, L.; Jiao, N.; Zhou, P.; Sun, L. Three-Dimensional Dirac Semimetal $\beta-\mathrm{PbO}_{2}$. Phys. Status Solidi 2017, 11, 1700271. 
(93) Chen, T.; Shao, D.; Lu, P.; Wang, X.; Wu, J.; Sun, J.; Xing, D. Anharmonic Effect driven Topological Phase Transition in $\mathrm{PbO}_{2}$ predicted by First-Principles Calculations. Phys. Rev. B 2018, 98, 144105.

(94) Gray, A. X.; Minár, J.; Ueda, S.; Stone, P. R.; Yamashita, Y.; Fujii, J.; Braun, J.; Plucinski, L.; Schneider, C. M.; Panaccione, G.; Ebert, H.; Dubon, O. D.; Kobayashi, K.; Fadley, C. S. Bulk Electronic Structure of the Dilute Magnetic Semiconductor $G a_{1-x} M n_{x} A s$ through Hard X-ray Angle-Resolved Photoemission. Nat. Mater. 2012, 11, 957-962.

(95) Gray, A. X. In Hard X-ray Angle Resolved Spectroscopy in Hard X-ray Photoelectron Spectroscopy (HAXPES), Springer Series in Surface Sciences; Woicik, J., Ed.; Springer International Publishing: Switzerland, 2016; Vol. 59.

(96) Takata, Y.; Kayanuma, Y.; Yabashi, M.; Tamasaku, K.; Nishino, Y.; Miwa, D.; Harada, Y.; Horiba, K.; Shin, S.; Tanaka, S.; Ikenaga, E.; Kobayashi, K.; Senba, Y.; Ohashi, H.; Ishikawa, T. Recoil Effects of Photoelectrons in a Solid. Phys. Rev. B 2007, 75, 233404.

(97) Dietl, T. A Ten-Year Perspective on Dilute Magnetic Semiconductors and Oxides. Nat. Mater. 2010, 9, 965-974.

(98) Braun, J.; Minár, J.; Mankovsky, S.; Strocov, V. N.; Brookes, N. B.; Plucinski, L.; Schneider, C. M.; Fadley, C. S.; Ebert, H. Exploring the XPS Limit in Soft and Hard X-ray Angle-Resolved Photoemission using a Temperature-Dependent one-step Theory. Phys. Rev. B 2013, 88, 205409.

(99) Keqi, A. et al. Electronic Structure of the Dilute Magnetic Semiconductor $G a_{1-x} M n_{x} P$ from Hard X-ray Photoelectron Spectroscopy and Angle-Resolved Photoemission. Phys. Rev. B 2018, 97, 155149. 
(100) Kobayashi, M.; Muneta, I.; Schmitt, T.; Patthey, L.; Ohya, S.; Tanaka, M.; Oshima, M.; Strocov, V. N. Digging up Bulk Band Dispersion buried under a Passivation Layer. Appl. Phys. Lett. 2012, 101, 242103.

(101) Papp, C.; Plucinski, L.; Minar, J.; Braun, J.; Ebert, H.; Schneider, C. M.; Fadley, C. S. Band Mapping in X-ray Photoelectron Spectroscopy: An Experimental and Theoretical Study of W(110) with $1.25 \mathrm{keV}$ excitation. Phys. Rev. B 2011, 84, 045433.

(102) Hasan, M. Z.; Kane, C. L. Colloquium: Topological insulators. Rev. Mod. Phys. 2010, 82, 3045-3067.

(103) Hasan, M. Z.; Moore, J. E. Three-Dimensional Topological Insulators. Annu. Rev. Condensed Matter Phys. 2011, 2, 55-78.

(104) Qi, X.-L.; Zhang, S.-C. Topological Insulators and Superconductors. Rev. Mod. Phys. 2011, 83, 1057-1110.

(105) Lv, B.; Qian, T.; Ding, H. Angle-resolved Photoemission Spectroscopy and its Application to Topological Materials. Nat. Rev. Phys. 2019, 1, 609-626.

(106) Xia, Y.; Qian, D.; Hsieh, D.; Wray, L.; Pal, A.; Lin, H.; Bansil, A.; Grauer, D.; Hor, Y. S.; Cava, R. J.; Hasan, M. Z. Observation of a Large-gap Topological-Insulator Class with a Single Dirac Cone on the Surface. Nat. Phys. 2009, 5, 398-402.

(107) Chen, Y. L.; Analytis, J. G.; Chu, J.-H.; Liu, Z. K.; Mo, S.-K.; Qi, X. L.; Zhang, H. J.; Lu, D. H.; Dai, X.; Fang, Z.; Zhang, S. C.; Fisher, I. R.; Hussain, Z.; Shen, Z.X. Experimental Realization of a Three-Dimensional Topological Insulator, $B i_{2} \mathrm{Te}_{3}$. Science 2009, 325, 178-181.

(108) Yan, B.; Felser, C. Topological Materials: Weyl Semimetals. Annu. Rev. Condensed Matter Phys. 2017, 8, 337-354. 
(109) Liu, Z. K.; Zhou, B.; Zhang, Y.; Wang, Z. J.; Weng, H. M.; Prabhakaran, D.; Mo, S.K.; Shen, Z. X.; Fang, Z.; Dai, X.; Hussain, Z.; Chen, Y. L. Discovery of a ThreeDimensional Topological Dirac Semimetal, $N a_{3}$ Bi. Science 2014, 343, 864-867.

(110) Neupane, M.; Xu, S.-Y.; Sankar, R.; Alidoust, N.; Bian, G.; Liu, C.; Belopolski, I.; Chang, T.-R.; Jeng, H.-T.; Lin, H.; Bansil, A.; Chou, F.; Hasan, M. Z. Observation of a Three-Dimensional Topological Dirac Semimetal Phase in High-Mobility $C d_{3} A s_{2}$. Nat. Commun. 2014, 5, 3786.

(111) Fu, L. Topological Crystalline Insulators. Phys. Rev. Lett. 2011, 106, 106802.

(112) Hsieh, T. H.; Lin, H.; Liu, J.; Duan, W.; Bansil, A.; Fu, L. Topological Crystalline Insulators in the SnTe Material Class. Nat. Commun. 2012, 3, 982.

(113) Fu, L.; Kane, C. L. Topological Insulators with Inversion Symmetry. Phys. Rev. B 2007, 76, 045302 .

(114) Murakami, S. Phase Transition between the Quantum Spin Hall and Insulator Phases in 3D: Emergence of a Topological Gapless Phase. New Journ. Phys. 2007, 9, 356-356.

(115) Moore, J. E. The Birth of Topological Insulators. Nature 2010, 464, 194-198.

(116) Moore, J. E.; Balents, L. Topological Invariants of Time-Reversal-Invariant Band Structures. Phys. Rev. B 2007, 75, 121306.

(117) Hsieh, D.; Xia, Y.; Wray, L.; Qian, D.; Pal, A.; Dil, J. H.; Osterwalder, J.; Meier, F.; Bihlmayer, G.; Kane, C. L.; Hor, Y. S.; Cava, R. J.; Hasan, M. Z. Observation of Unconventional Quantum Spin Textures in Topological Insulators. Science 2009, 323, $919-922$.

(118) Wang, Y. H.; Hsieh, D.; Pilon, D.; Fu, L.; Gardner, D. R.; Lee, Y. S.; Gedik, N. Observation of a Warped Helical Spin Texture in $\mathrm{Bi}_{2} \mathrm{Se}_{3}$ from Circular Dichroism Angle-Resolved Photoemission Spectroscopy. Phys. Rev. Lett. 2011, 10\%, 207602. 
(119) Hsieh, D.; Qian, D.; Wray, L.; Xia, Y.; Hor, Y. S.; Cava, R. J.; Hasan, M. Z. A Topological Dirac Insulator in a Quantum Spin Hall Phase. Nature 2008, 452, 970974.

(120) Scanlon, D. O.; King, P. D. C.; Singh, R. P.; de la Torre, A.; Walker, S. M.; Balakrishnan, G.; Baumberger, F.; Catlow, C. R. A. Controlling Bulk Conductivity in Topological Insulators: Key Role of Anti-Site Defects. Adv. Mater. 2012, 24, 21542158.

(121) Tokura, Y.; Yasuda, K.; Tsukazaki, A. Magnetic Topological Insulators. Nat. Rev. Phys. 2019, 1, 126-143.

(122) Sato, M.; Ando, Y. Topological Superconductors: a Review. Rep. Prog. Phys. 2017, $80,076501$.

(123) Dzero, M.; Xia, J.; Galitski, V.; Coleman, P. Topological Kondo Insulators. Annu. Rev. Condensed Matter Phys 2016, 7, 249-280.

(124) Rashba, E. I. Properties of Semiconductors with an Extremum Loop .1. Cyclotron and Combinational Resonance in a Magnetic Field perpendicular to the Plane of the Loop. Sov. Phys. Solid State 1960, 17, 1109-1122.

(125) Bychkov, Y. A.; Rashba, E. I. Properties of a 2D Electron Gas with Lifted Spectral Degeneracy. JETP Lett. 1984, 39, 78-81.

(126) Bihlmayer, G.; Rader, O.; Winkler, R. Focus on the Rashba Effect. New J. Phys. 2015, 17, 050202.

(127) Di Sante, D.; Barone, P.; Bertacco, R.; Picozzi, S. Electric Control of the Giant Rashba Effect in Bulk GeTe. Adv. Mater. 2013, 25, 509-513.

(128) Picozzi, S. Ferroelectric Rashba Semiconductors as a Novel Class of Multifunctional Materials. Front. Phys. 2014, 2, A010, 1-5. 
(129) LaShell, S.; McDougall, B. A.; Jensen, E. Spin Splitting of an Au(111) Surface State Band Observed with Angle Resolved Photoelectron Spectroscopy. Phys. Rev. Lett. 1996, 77, 3419-3422.

(130) Ast, C. R.; Henk, J.; Ernst, A.; Moreschini, L.; Falub, M. C.; Pacilé, D.; Bruno, P.; Kern, K.; Grioni, M. Giant Spin Splitting through Surface Alloying. Phys. Rev. Lett. 2007, 98, 186807.

(131) Hochstrasser, M.; Tobin, J. G.; Rotenberg, E.; Kevan, S. D. Spin-Resolved Photoemission of Surface States of W(110)-1x1-H. Phys. Rev. Lett. 2002, 89, 216802.

(132) Koroteev, Y. M.; Bihlmayer, G.; Gayone, J. E.; Chulkov, E. V.; Blügel, S.; Echenique, P. M.; Hofmann, P. Strong Spin-Orbit Splitting on Bi Surfaces. Phys. Rev. Lett. 2004, 93, 046403.

(133) Ishizaka, K. et al. Giant Rashba-type Spin Splitting in Bulk BiTeI. Nat. Mater. 2011, $10,521-526$.

(134) Crepaldi, A.; Moreschini, L.; Autès, G.; Tournier-Colletta, C.; Moser, S.; Virk, N.; Berger, H.; Bugnon, P.; Chang, Y. J.; Kern, K.; Bostwick, A.; Rotenberg, E.; Yazyev, O. V.; Grioni, M. Giant Ambipolar Rashba Effect in the Semiconductor BiTeI. Phys. Rev. Lett. 2012, 109, 096803.

(135) Nitta, J.; Akazaki, T.; Takayanagi, H.; Enoki, T. Gate Control of Spin-Orbit Interaction in an Inverted $\operatorname{In}_{0.53} \mathrm{Ga}_{0.47} \mathrm{As} / \mathrm{In}_{0.52} \mathrm{Al}_{0.48}$ As Heterostructure. Phys. Rev. Lett. 1997, 78, 1335-1338.

(136) Bawden, L. et al. Hierarchical Spin-Orbital Polarization of a Giant Rashba system. Sci. Adv. 2015, 1, e1500495.

(137) Zhang, P.; Richard, P.; Qian, T.; Xu, Y.-M.; Dai, X.; Ding, H. A precise method for 
visualizing dispersive features in image plots. Review of Scientific Instruments 2011, 82, 043712.

(138) Liebmann, M. et al. Giant Rashba-Type Spin Splitting in Ferroelectric GeTe(111). Adv. Mater. 2016, 28, 560-565.

(139) Chattopadhyay, T.; Boucherle, J. X.; vonSchnering, H. G. Neutron Diffraction Study on the Structural Phase Transition in GeTe. J. Phys. C: Solid State Phys. 1987, 20, $1431-1440$.

(140) Wang, R.; Boschker, J. E.; Bruyer, E.; Sante, D. D.; Picozzi, S.; Perumal, K.; Giussani, A.; Riechert, H.; Calarco, R. Toward Truly Single Crystalline GeTe Films: The Relevance of the Substrate Surface. J. Phys. Chem. 2014, 118, 29724-29730.

(141) Krempaský, J. et al. Disentangling Bulk and Surface Rashba Effects in Ferroelectric $\alpha$-GeTe. Phys. Rev. B 2016, 94, 205111.

(142) Manzeli, S.; Ovchinnikov, D.; Pasquier, D.; Yazyev, O. V.; Kis, A. 2D Transition Metal Dichalcogenides. Nat. Rev. Mater 2017, 2, 17033.

(143) Chhowalla, M.; Shin, H. S.; Eda, G.; Li, L.-J.; Loh, K. P.; Zhang, H. The Chemistry of Two-dimensional Layered Transition Metal Dichalcogenide Nanosheets. Nat. Chemistry 2013, 5, 263-275.

(144) Zhang, J.; Jia, S.; Kholmanov, I.; Dong, L.; Er, D.; Chen, W.; Guo, H.; Jin, Z.; Shenoy, V. B.; Shi, L.; Lou, J. Monolayer Transition-Metal Dichalcogenides. ACS Nano 2017, 11, 8192-8198.

(145) Wang, Q. H.; Kalantar-Zadeh, K.; Kis, A.; Coleman, J. N.; Strano, M. S. Electronics and Optoelectronics of Two-Dimensional Transition Metal Dichalcogenides. Na. Nanotechn. 2012, 7, 699-712. 
(146) Xu, X.; Yao, W.; Xiao, D.; Heinz, T. F. Spin and Pseudospins in Layered Transition Metal Dichalcogenides. Nat. Phys. 2014, 10, 343-350.

(147) Morosan, E.; Zandbergen, H. W.; Dennis, B. S.; Bos, J. W. G.; Onose, Y.; Klimczuk, T.; Ramirez, A. P.; Ong, N. P.; Cava, R. J. Superconductivity in $C u_{x} T i S e_{2}$. Nat. Phys. 2006, 2, 544-550.

(148) Borisenko, S. V.; Kordyuk, A. A.; Yaresko, A. N.; Zabolotnyy, V. B.; Inosov, D. S.; Schuster, R.; Büchner, B.; Weber, R.; Follath, R.; Patthey, L.; Berger, H. Pseudogap and Charge Density Waves in Two Dimensions. Phys. Rev. Lett. 2008, 100, 196402.

(149) Mak, K. F.; Lee, C.; Hone, J.; Shan, J.; Heinz, T. F. Atomically Thin $M o S_{2}$ : A New Direct-Gap Semiconductor. Phys. Rev. Lett. 2010, 105, 136805.

(150) Xiao, D.; Liu, G.-B.; Feng, W.; Xu, X.; Yao, W. Coupled Spin and Valley Physics in Monolayers of $\mathrm{MoS}_{2}$ and Other Group-VI Dichalcogenides. Phys. Rev. Lett. 2012, $108,196802$.

(151) Ugeda, M. M.; Bradley, A. J.; Shi, S.-F.; da Jornada, F. H.; Zhang, Y.; Qiu, D. Y.; Ruan, W.; Mo, S.-K.; Hussain, Z.; Shen, Z.-X.; Wang, F.; Louie, S. G.; Crommie, M. F. Giant Bandgap Renormalization and Excitonic Effects in a Monolayer Transition Metal Dichalcogenide Semiconductor. Nat. Mater. 2014, 13, 1091-1095.

(152) Luo, H.; Xie, W.; Tao, J.; Inoue, H.; Gyenis, A.; Krizan, J. W.; Yazdani, A.; Zhu, Y.; Cava, R. J. Polytypism, Polymorphism, and Superconductivity in $\operatorname{TaSe}_{2-x} T e_{x}$. Proc. Natl. Acad. Sci. U.S.A. 2015, 112, E1174-E1180.

(153) Li, L. J.; O’Farrell, E. C. T.; Loh, K. P.; Eda, G.; Özyilmaz, B.; Castro Neto, A. H. Controlling Many-Body States by the Electric-Field Effect in a Two-dimensional Material. Nature 2015, 529, 185-189. 
(154) Soluyanov, A. A.; Gresch, D.; Wang, Z.; Wu, Q.; Troyer, M.; Dai, X.; Bernevig, B. A. Type-II Weyl Semimetals. Nature 2015, 527, 495-498.

(155) Bahramy, M. et al. Ubiquitous Formation of Bulk Dirac Cones and Topological Surface States from a Single Orbital Manifold in Transition-Metal Dichalcogenides. Nat. Mater. 2017, 17, 21-28.

(156) Suga, S.; Tusche, C.; Matsushita, Y.-i.; Ellguth, M.; Irizawa, A.; Kirschner, J. Momentum Microscopy of the Layered Semiconductor $T i S_{2}$ and Ni intercalated $N i_{1 / 3} T i S_{2}$. New J. Phys. 2015, 17, 083010.

(157) Chen, P.; Pai, W. W.; Chan, Y.-H.; Takayama, A.; Xu, C.-Z.; Karn, A.; Hasegawa, S.; Chou, M. Y.; Mo, S.-K.; Fedorov, A.-V.; Chiang, T.-C. Emergence of Charge Density Waves and a Pseudogap in Single-layer TiTe 2 . Nat. Commun. 2017, 8, 516.

(158) Watson, M. D.; Clark, O. J.; Mazzola, F.; Marković, I.; Sunko, V.; Kim, T. K.; Rossnagel, K.; King, P. D. Orbital and $k_{z}$ Selective Hybridization of Se 4p and Ti 3d States in the Charge Density Wave Phase of TiSe $e_{2}$ Phys. Rev. Lett. 2019, 122, 076404.

(159) Katsuyasu, K.; Takahashi, J.; Nakata, R.; Sumita, M. Temperature Dependence of Resistivities in $\mathrm{TiS}_{2}$ and Its Cu-Intercalate. J. Phys. Soc. Jpn. 1989, 58, 949-955.

(160) Di Salvo, F. J.; Moncton, D. E.; Waszczak, J. V. Electronic Properties and Superlattice Formation in the Semimetal TiSe 2 . Phys. Rev. B 1976, 14, 4321-4328.

(161) Rajaji, V.; Dutta, U.; Sreeparvathy, P. C.; Sarma, S. C.; Sorb, Y. A.; Joseph, B.; Sahoo, S.; Peter, S. C.; Kanchana, V.; Narayana, C. Structural, Vibrational, and Electrical Properties of $1 \mathrm{~T}-\mathrm{TiTe}_{2}$ under Hydrostatic Pressure: Experiments and Theory. Phys. Rev. B 2018, 97, 085107. 
(162) Watson, M. D.; Beales, A. M.; King, P. D. C. On the Origin of the Anomalous Peak in the Resistivity of TiSe2. Phys. Rev. B 2019, 99, 195142.

(163) Doublet, M.-L.; Gallego-Planas, N.; Philipsen, P. H. T.; Brec, R.; Jobic, S. A New Theoretical Approach for the Electrical Properties of $\operatorname{TiX}_{2}(X=S, S e, T e)$ Phases with Density Functional Calculations. J. Chem. Phys. 1998, 108, 649-658.

(164) Kidd, T. E.; Miller, T.; Chou, M. Y.; Chiang, T.-C. Electron-Hole Coupling and the Charge Density Wave Transition in TiSe ${ }_{2}$. Phys. Rev. Lett. 2002, 88, 226402.

(165) Cercellier, H.; Monney, C.; Clerc, F.; Battaglia, C.; Despont, L.; Garnier, M. G.; Beck, H.; Aebi, P.; Patthey, L.; Berger, H.; Forró, L. Evidence for an Excitonic Insulator Phase in 1T-TiSe 2 . Phys. Rev. Lett. 2007, 99, 146403.

(166) Zhao, J. F.; Ou, H. W.; Wu, G.; Xie, B. P.; Zhang, Y.; Shen, D. W.; Wei, J.; Yang, L. X.; Dong, J. K.; Arita, M.; Namatame, H.; Taniguchi, M.; Chen, X. H.;

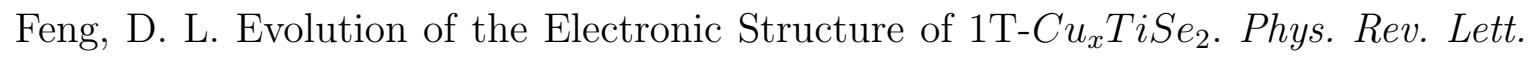
2007, 99, 146401.

(167) Rasch, J. C. E.; Stemmler, T.; Müller, B.; Dudy, L.; Manzke, R. 1T-TiSe 2 : Semimetal or Semiconductor? Phys. Rev. Lett. 2008, 101, 237602.

(168) Chen, P.; Chan, Y.-H.; Fang, X.-Y.; Mo, S.-K.; Hussain, Z.; Fedorov, A.-V.; Chou, M. Y.; Chiang, T.-C. Hidden Order and Dimensional Crossover of the Charge Density Waves in TiSe $e_{2}$ Sci. Rep. 2016, 6, 37910.

(169) Kogar, A.; Rak, M. S.; Vig, S.; Husain, A. A.; Flicker, F.; Joe, Y. I.; Venema, L.; MacDougall, G. J.; Chiang, T. C.; Fradkin, E.; Wezel, J. v.; Abbamonte, P. Signatures of Exciton Condensation in a Transition Metal Dichalcogenide. Science 2017, 358, 1314-1317. 
(170) Rahn, D. J.; Hellmann, S.; Kalläene, M.; Sohrt, C.; Kim, T. K.; Kipp, L.; Rossnagel, K. Gaps and Kinks in the Electronic Structure of the Superconductor $2 \mathrm{H}-\mathrm{NbSe}_{2}$ from Angle-Resolved Photoemission at 1 K. Phys. Rev. B 2012, 85, 224532.

(171) Wilson, J. A.; Di Salvo, F. J.; Mahajan, S. Charge-Density Waves in Metallic, Layered, Transition-Metal Dichalcogenides. Phys. Rev. Lett. 1974, 32, 882-885.

(172) Johannes, M. D.; Mazin, I. I.; Howells, C. A. Fermi-surface nesting and the origin of the charge-density wave in $\mathrm{NbSe}_{2}$. Phys. Rev. B 2006, 73, 205102.

(173) Inosov, D. S.; Zabolotnyy, V. B.; Evtushinsky, D. V.; Kordyuk, A. A.; BÃAchner, B.; Follath, R.; Berger, H.; Borisenko, S. V. Fermi Surface Nesting in several Transition Metal Dichalcogenides. New J. Phys. 2008, 10, 125027.

(174) Feng, Y.; van Wezel, J.; Wang, J.; Flicker, F.; Silevitch, D. M.; Littlewood, P. B.; Rosenbaum, T. F. Itinerant Density Wave Instabilities at Classical and Quantum Critical Points. Nat. Phys. 2015, 11, 865-871.

(175) Flicker, F.; van Wezel, J. Charge Order from Orbital-dependent Coupling Evidenced by $\mathrm{NbSe}_{2}$. Nat. Commun. 2015, 6, 7034 .

(176) Biswas, D. et al. Narrow-band Anisotropic Electronic Structure of Re $S_{2}$. Phys. Rev. $B$ 2017, 96, 085205.

(177) Clark, O. J.; Mazzola, F.; Feng, J.; Sunko, V.; Marković, I.; Bawden, L.; Kim, T. K.; King, P. D. C.; Bahramy, M. S. Dual Quantum Confinement and Anisotropic Spin Splitting in the Multivalley Semimetal PtSe . Phys. Rev. B 2019, 99, 045438.

(178) Kertesz, M.; Hoffmann, R. Octahedral vs. Trigonal-Prismatic Coordination and Clustering in Transition-Metal Dichalcogenides. J. Am. Chem. Soc. 1984, 106, 3453-3460.

(179) Tongay, S. et al. Monolayer Behaviour in Bulk $R e S_{2}$ due to Electronic and Vibrational Decoupling. Nat. Commun. 2014, 5, 3252. 
(180) Hart, L. S.; Webb, J. L.; Dale, S.; Bending, S. J.; Mucha-Kruczynski, M.; Wolverson, D.; Chen, C.; Avila, J.; Asensio, M. C. Electronic Bandstructure and van der Waals Coupling of $\mathrm{ReSe}_{2}$ Revealed by High-Resolution Angle-Resolved Photoemission Spectroscopy. Sci. Rep. 2017, 7, 5145.

(181) Gehlmann, M. et al. Direct Observation of the Band Gap Transition in Atomically Thin $\operatorname{ReS}_{2}$. Nano Lett. 2017, 17, 5187-5192.

(182) Friemelt, K.; LuxâSteiner, M.; Bucher, E. Optical Properties of the Layered Transition Metal Dichalcogenide $R e S_{2}$ : Anisotropy in the van der Waals Plane. J. Appl. Phys. 1993, 74, 5266-5268.

(183) Ho, C.; Hsieh, M.; Wu, C.; Huang, Y.; Tiong, K. Dichroic Optical and Electrical Properties of Rhenium Dichalcogenides Layer Compounds. J. Alloys and Compounds 2007, 442, 245-248.

(184) Splendiani, A.; Sun, L.; Zhang, Y.; Li, T.; Kim, J.; Chim, C.-Y.; Galli, G.; Wang, F. Emerging Photoluminescence in Monolayer MoS . Nano Lett. 2010, 10, 1271-1275.

(185) Zhang, Y. et al. Direct observation of the Transition from Indirect to Direct Bandgap in Atomically Thin Epitaxial $\mathrm{MoSe}_{2}$. Nat. Nanotechn. 2014, 9, 111-115.

(186) Riley, J. M.; Meevasana, W.; Bawden, L.; Asakawa, M.; Takayama, T.; Eknapakul, T.; Kim, T. K.; Hoesch, M.; Mo, S.-K.; Takagi, H.; Sasagawa, T.; Bahramy, M. S.; King, P. D. C. Negative Electronic Compressibility and Tunable Spin Splitting in $\mathrm{WSe}_{2}$. Nat. Nanotechn. 2015, 10, 1043-1047.

(187) Miwa, J. A.; Ulstrup, S.; Sørensen, S. G.; Dendzik, M.; Grubišić Čabo, A.; Bianchi, M.; Lauritsen, J. V.; Hofmann, P. Electronic Structure of Epitaxial Single-Layer $\mathrm{MoS}_{2}$. Phys. Rev. Lett. 2015, 114, 046802. 
(188) Eknapakul, T.; King, P. D. C.; Asakawa, M.; Buaphet, P.; He, R.-H.; Mo, S.-K.; Takagi, H.; Shen, K. M.; Baumberger, F.; Sasagawa, T.; Jungthawan, S.; Meevasana, W. Electronic Structure of a Quasi-Freestanding $M o S_{2}$ Monolayer. Nano Lett. 2014, 14 , $1312-1316$.

(189) Kang, M.; Kim, B.; Ryu, S. H.; Jung, S. W.; Kim, J.; Moreschini, L.; Jozwiak, C.; Rotenberg, E.; Bostwick, A.; Kim, K. S. Universal Mechanism of Band-Gap Engineering in Transition-Metal Dichalcogenides. Nano Lett. 2017, 17, 1610-1615.

(190) Grubišić Čabo, A.; Miwa, J. A.; Grønborg, S. S.; Riley, J. M.; Johannsen, J. C.; Cacho, C.; Alexander, O.; Chapman, R. T.; Springate, E.; Grioni, M.; Lauritsen, J. V.; King, P. D. C.; Hofmann, P.; Ulstrup, S. Observation of Ultrafast Free Carrier Dynamics in Single Layer $M o S_{2}$. Nano Lett. 2015, 15, 5883-5887.

(191) Bertoni, R.; Nicholson, C.; Waldecker, L.; Hübener, H.; Monney, C.; De Giovannini, U.; Puppin, M.; Hoesch, M.; Springate, E.; Chapman, R.; Cacho, C.; Wolf, M.; Rubio, A.; Ernstorfer, R. Generation and Evolution of Spin-, Valley-, and Layer-Polarized Excited Carriers in Inversion-Symmetric WSe 2 . Phys. Rev. Lett. 2016, 117, 277201.

(192) Ulstrup, S.; Grubišić Čabo, A.; Biswas, D.; Riley, J. M.; Dendzik, M.; Sanders, C. E.; Bianchi, M.; Cacho, C.; Matselyukh, D.; Chapman, R. T.; Springate, E.; King, P. D. C.; Miwa, J. A.; Hofmann, P. Spin and Valley Control of Free Carriers in Singlelayer $W S_{2}$. Phys. Rev. B 2017, 95, 041405.

(193) Ulstrup, S.; Grubišić Čabo, A.; Miwa, J. A.; Riley, J. M.; Grønborg, S. S.; Johannsen, J. C.; Cacho, C.; Alexander, O.; Chapman, R. T.; Springate, E.; Bianchi, M.; Dendzik, M.; Lauritsen, J. V.; King, P. D. C.; Hofmann, P. Ultrafast Band Structure Control of a Two-Dimensional Heterostructure. ACS Nano 2016, 10, 6315-6322.

(194) Nguyen, P. V.; Teutsch, N. C.; Wilson, N. P.; Kahn, J.; Xia, X.; Graham, A. J.; Kandyba, V.; Giampietri, A.; Barinov, A.; Constantinescu, G. C.; Yeung, N.; Hine, N. 
D. M.; Xu, X.; Cobden, D. H.; Wilson, N. R. Visualizing Electrostatic Gating Effects in Two-Dimensional Heterostructures. Nature 2019, 572, 220-223.

(195) Strocov, V. N.; Shi, M.; Kobayashi, M.; Monney, C.; Wang, X.; Krempasky, J.; Schmitt, T.; Patthey, L.; Berger, H.; Blaha, P. Three-Dimensional Electron Realm in $V \mathrm{Se}_{2}$ by Soft-X-Ray Photoelectron Spectroscopy: Origin of Charge-Density Waves. Phys. Rev. Lett. 2012, 109, 086401.

(196) Feng, J. et al. Electronic Structure and Enhanced Charge-Density Wave Order of Monolayer $\mathrm{VSe}_{2}$. Nano Lett. 2018, 18, 4493-4499.

(197) Tsutsumi, K. X-ray-diffraction Study of the Periodic Lattice Distortion associated with a Charge-Density-Wave in 1T-VSe $e_{2}$. Phys. Rev. B 1982, 26, 5756-5759.

(198) Eaglesham, D. J.; Withers, R. L.; Bird, D. M. Charge-Density-Wave Transitions in 1T-VSe $e_{2}$. J. Phys. C: Solid State Phys. 1986, 19, 359-367.

(199) Duvjir, G. et al. Emergence of a Metal-Insulator Transition and High-Temperature Charge-Density Waves in $\mathrm{VSe}_{2}$ at the Monolayer Limit. Nano Lett. 2018, 18, 54325438.

(200) Chen, P.; Pai, W. W.; Chan, Y.-H.; Madhavan, V.; Chou, M.; Mo, S.-K.; Fedorov, A.V.; Chiang, T.-C. Unique Gap Structure and Symmetry of the Charge Density Wave in Single-Layer $V S e_{2}$. Phys. Rev. Lett. 2018, 121, 196402.

(201) Bonilla, M.; Kolekar, S.; Ma, Y.; Diaz, H. C.; Kalappattil, V.; Das, R.; Eggers, T.; Gutierrez, H. R.; Phan, M.-H.; Batzill, M. Strong Room-Temperature Ferromagnetism in $V_{S e_{2}}$ Monolayers on van der Waals Substrates. Nature Nanotechnol. 2018, 13, 289293.

(202) Zhang, W.; Zhang, L.; Wong, P. K. J.; Yuan, J.; Vinai, G.; Torelli, P.; van der 
Laan, G.; Feng, Y. P.; Wee, A. T. S. Magnetic Transition in Monolayer $\mathrm{VSe}_{2}$ via Interface Hybridization. ACS Nano 2019, 13, 8997-9004.

(203) Vinai, G.; Bigi, C.; Rajan, A.; Watson, M. D.; Lee, T.-L.; Mazzola, F.; Modesti, S.; Barua, S.; Ciomaga Hatnean, M.; Balakrishnan, G.; King, P. D. C.; Torelli, P.; Rossi, G.; Panaccione, G. Proximity-induced Ferromagnetism and Chemical Reactivity in Few-Layer $\mathrm{VSe}_{2}$ Heterostructures. Phys. Rev. B 2020, 101, 035404.

(204) Zeng, H.; Dai, J.; Yao, W.; Xiao, D.; Cui, X. Valley Polarization in $M o S_{2}$ Monolayers by Optical Pumping. Nat. Nanotechnol. 2012, 7, 490-493.

(205) Mak, K. F.; He, K.; Shan, J.; Heinz, T. F. Control of Valley Polarization in Monolayer $M_{o S}$ by Optical Helicity. Nat. Nanotechnol. 2012, 7, 494-498.

(206) Mak, K. F.; McGill, K. L.; Park, J.; McEuen, P. L. The valley Hall Effect in $M o S_{2}$ Transistors. Science 2014, 344, 1489-1492.

(207) Jones, A. M.; Yu, H.; Ross, J. S.; Klement, P.; Ghimire, N. J.; Yan, J.; Mandrus, D. G.; Yao, W.; Xu, X. Spin-layer Locking Effects in Optical Orientation of Exciton Spin in Bilayer $\mathrm{WSe}_{2}$. Nat. Phys. 2014, 10, 130-134.

(208) Zhang, X.; Liu, Q.; Luo, J.-W.; Freeman, A. J.; Zunger, A. Hidden Spin-Polarization in Inversion-Symmetric Bulk Crystals. Nat. Phys. 2014, 10, 387-393.

(209) Bana, H. et al. Epitaxial growth of single-orientation high-quality $M o S_{2}$ monolayers. 2D Mater. 2018, 5, 035012.

(210) Bawden, L. et al. Spin-valley Locking in the Normal State of a Transition-Metal Dichalcogenide Superconductor. Nat. Commun. 2016, 7, 11711.

(211) Xi, X.; Wang, Z.; Zhao, W.; Park, J.-H.; Law, K. T.; Berger, H.; Forró, L.; Shan, J.; Mak, K. F. Ising Pairing in Superconducting $\mathrm{NbSe}_{2}$ Atomic Layers. Nat. Phys. 2016, 12, 139-143. 
(212) Clark, O. J.; Mazzola, F.; Marković, I.; Riley, J. M.; Feng, J.; Yang, B.-J.; Sumida, K.; Okuda, T.; Fujii, J.; Vobornik, I.; Kim, T. K.; Okawa, K.; Sasagawa, T.; Bahramy, M. S.; King, P. D. C. A General Route to form Topologically-Protected Surface and Bulk Dirac Fermions along High-Symmetry Lines. Electron. Struct. 2019, 1,014002 .

(213) Yan, L. et al. Identification of Topological Surface State in $P d T e_{2}$ Superconductor by Angle-Resolved Photoemission Spectroscopy. Chinese Phys. Lett. 2015, 32, 067303.

(214) Huang, H.; Zhou, S.; Duan, W. Type-II Dirac Fermions in the PtSe $e_{2}$ Class of Transition Metal Dichalcogenides. Phys. Rev. B 2016, 94, 121117.

(215) Noh, H.-J.; Jeong, J.; Cho, E.-J.; Kim, K.; Min, B.; Park, B.-G. Experimental Realization of Type-II Dirac Fermions in a PdTe 2 Superconductor. Phys. Rev. Lett. 2017, 119, 016401.

(216) Fei, F.; Bo, X.; Wang, R.; Wu, B.; Jiang, J.; Fu, D.; Gao, M.; Zheng, H.; Chen, Y.; Wang, X.; Bu, H.; Song, F.; Wan, X.; Wang, B.; Wang, G. Nontrivial Berry Phase and Type-II Dirac Transport in the Layered Material PdTe 2. Phys. Rev. B 2017, 96, 041201.

(217) Yan, M. et al. Lorentz-violating Type-II Dirac Fermions in Transition Metal Dichalcogenide PtTe 2 . Nat. Commun. 2017, 8, 257.

(218) Clark, O. J. et al. Fermiology and Superconductivity of Topological Surface States in PdTe $e_{2}$. Phys. Rev. Lett. 2018, 120, 156401.

(219) Xu, C.; Li, B.; Jiao, W.; Zhou, W.; Qian, B.; Sankar, R.; Zhigadlo, N. D.; Qi, Y.; Qian, D.; Chou, F.-C.; Xu, X. Topological Type-II Dirac Fermions Approaching the Fermi Level in a Transition Metal Dichalcogenide $\mathrm{NiTe}_{2}$. Chem. Mater. 2018, 30, $4823-4830$. 
(220) Fei, F. et al. Band Structure Perfection and Superconductivity in Type-II Dirac Semimetal $\operatorname{Ir}_{1-x} P t_{x} T e_{2}$. Adv. Mater. 2018, 30, 1801556.

(221) Cucchi, I.; Gutiérrez-Lezama, I.; Cappelli, E.; McKeown Walker, S.; Bruno, F. Y.; Tenasini, G.; Wang, L.; Ubrig, N.; Barreteau, C.; Giannini, E.; Gibertini, M.; Tamai, A.; Morpurgo, A. F.; Baumberger, F. Microfocus Laser-Angle-Resolved Photoemission on Encapsulated Mono-, Bi-, and Few-Layer $1 T^{\prime}-W T e_{2}$. Nano Lett. 2019, $19,554-560$.

(222) Tang, S. et al. Quantum Spin Hall State in Monolayer $1 T^{\prime}-W T e_{2}$. Nat. Phys. 2017, 13, 683-687.

(223) Cox, P. A. Transition Metal Oxides: An Introduction to Their Electronic Structure and Properties, 10th ed.; Oxford University Press: UK, 2010.

(224) Cox, P. A. In The Electronic Structure of Transition Metal Oxides and Chalcogenides, in Physics and Chemistry of Low-Dimensional Inorganic Conductors. NATO ASI Series (Series B: Physics); Schlenker, C., Dumas, J., Greenblatt, M., van Smaalen, S., Eds.; Springer: Boston (MA), 1996; Vol. 266.

(225) Tokura, Y.; Nagaosa, N. Orbital Physics in Transition-Metal Oxides. Science 2000, 288, 462-467.

(226) Samarth, N. Quantum Materials Discovery from a Synthesis Perspective. Nat. Mater. 2017, 16, 1068-1076.

(227) Basov, D. N.; Averitt, R. D.; Hsieh, D. Towards Properties on Demand in Quantum Materials. Nat. Mater. 2017, 16, 1077-1088.

(228) Rondinelli, J. M.; May, S. J. Oxide Interfaces: Instrumental Insights. Nat. Mater. 2012, 11, 833-834. 
(229) Biesinger, M. C.; Lau, L. W.; Gerson, A. R.; Smart, R. S. C. Resolving Surface Chemical States in XPS Analysis of first Row Transition Metals, Oxides and Hydroxides: Sc, Ti, V, Cu and Zn. Appl. Surf. Sci. 2010, 257, 887-898.

(230) Morosan, E.; Natelson, D.; Nevidomskyy, A. H.; Si, Q. Strongly Correlated Materials. Adv. Mater. 2012, 24, 4896-4923.

(231) Sawatzky, G. A.; Allen, J. W. Magnitude and Origin of the Band Gap in NiO. Phys. Rev. Lett. 1984, 53, 2339-2342.

(232) Zaanen, J.; Sawatzky, G. A.; Allen, J. W. Band Gaps and Electronic Structure of Transition-Metal Compounds. Phys. Rev. Lett. 1985, 55, 418-421.

(233) Henderson, M. A. A Surface Science Perspective on $\mathrm{TiO}_{2}$ Photocatalysis. Surf. Sci. Rep 2011, 66, 185-297.

(234) Chen, X.; Mao, S. S. Titanium Dioxide Nanomaterials: Synthesis, Properties, Modifications, and Applications. Chem. Rev. 2007, 107, 2891-2959.

(235) Diebold, U. The Surface Science of Titanium Dioxide. Surf. Sci. Rep. 2003, 48, 53229.

(236) Fujishima, A.; Zhang, X.; Tryk, D. A. TiO 2 Photocatalysis and Related Surface Phenomena. Surf. Sci. Rep. 2008, 63, 515-582.

(237) Di Valentin, C.; Pacchioni, G.; Selloni, A. Reduced and n-type Doped $\mathrm{TiO}_{2}$ : Nature of $T i^{3+}$ species. J. Phys. Chem. C 2009, 113, 20543-20552.

(238) Gabel, J.; Zapf, M.; Scheiderer, P.; Schütz, P.; Dudy, L.; Stübinger, M.; Schlueter, C.; Lee, T.-L.; Sing, M.; Claessen, R. Disentangling specific versus generic Doping Mechanisms in Oxide Heterointerfaces. Phys. Rev. B 2017, 95, 195109.

(239) Chambers, S. A.; Du, Y.; Zhu, Z.; Wang, J.; Wahila, M. J.; Piper, L. F.; Prakash, A.; Yue, J.; Jalan, B.; Spurgeon, S. R.; Kepaptsoglou, D. M.; Ramasse, Q. M.; 
Sushko, P. V. Interconversion of Intrinsic Defects in $\mathrm{SrTiO}_{3}(001)$. Phys. Rev. B 2018, $97,245204$.

(240) Yin, W. J.; Wen, B.; Zhou, C.; Selloni, A.; Liu, L. M. Excess Electrons in Reduced Rutile and Anatase $\mathrm{TiO}_{2}$. Surf. Sci. Rep. 2018, 73, 58-82.

(241) Wang, Z.; Wen, B.; Hao, Q.; Liu, L. M.; Zhou, C.; Mao, X.; Lang, X.; Yin, W. J.; Dai, D.; Selloni, A.; Yang, X. Localized Excitation of $T i^{3+}$ Ions in the Photoabsorption and Photocatalytic Activity of Reduced Rutile $\mathrm{TiO}_{2}$. J. Am. Chem. Soc. 2015, 137, 9146-9152.

(242) Salluzzo, M.; Cezar, J.; Brookes, N.; Bisogni, V.; De Luca, G.; Richter, C.; Thiel, S.; Mannhart, J.; Huijben, M.; Brinkman, A., et al. Orbital Reconstruction and the TwoDimensional Electron Gas at the $\mathrm{LaAlO}_{3} / \mathrm{SrTiO}_{3}$ interface. Phys. Rev. Lett. 2009, 102, 166804.

(243) Husanu, M.; Vaz, C. A. F. Spectroscopy of Complex Oxide Interfaces; Springer International Publishing, 2018; Vol. 266; pp 245-281.

(244) Strocov, V. N.; Kobayashi, M.; Wang, X.; Lev, L. L.; Krempasky, J.; Rogalev, V. V.; Schmitt, T.; Cancellieri, C.; Reinle-Schmitt, M. L. Soft-X-ray ARPES at the Swiss Light Source: From 3D Materials to Buried Interfaces and Impurities. Synchr. Rad. News 2014, 27, 31-40.

(245) Strocov, N. V.; Cancellieri, C.; Mishchenko, S. A. In Electrons and Polarons at Oxide Interfaces Explored by Soft-X-Ray ARPES, in Spectroscopy of Complex Oxide Interfaces; Cancellieri, C., Strocov, V., Eds.; Springer International Publishing: Boston (MA), 2018; Vol. 266.

(246) McKeown Walker, S.; Bruno, F. Y.; Wang, Z.; de la Torre, A.; Riccò, S.; Tamai, A.; Kim, T. K.; Hoesch, M.; Shi, M.; Bahramy, M. S.; King, P. D. C.; Baumberger, F. 
Carrier-Density Control of the $\mathrm{SrTiO}_{3}$ (001) Surface 2D Electron Gas studied by ARPES. Adv. Mater. 2015, 27, 3894-3899.

(247) King, P. D. C.; McKeown Walker, S.; Tamai, A.; de la Torre, A.; Eknapakul, T.; Buaphet, P.; Mo, S.-K.; Meevasana, W.; Bahramy, M. S.; Baumberger, F. Quasiparticle Dynamics and Spin-Orbital Texture of the $\mathrm{SrTiO}_{3}$ Two-Dimensional Electron Gas. Nat. Commun. 2014, 5, 3414.

(248) Berner, G.; Sing, M.; Fujiwara, H.; Yasui, A.; Saitoh, Y.; Yamasaki, A.; Nishitani, Y.; Sekiyama, A.; Pavlenko, N.; Kopp, T.; Richter, C.; Mannhart, J.; Suga, S.; Claessen, R. Direct k -Space Mapping of the Electronic Structure in an Oxide-Oxide Interface. Phys. Rev. Lett. 2013, 110, 247601.

(249) Koitzsch, A.; Ocker, J.; Knupfer, M.; Dekker, M. C.; Dörr, K.; Büchner, B.; Hoffmann, P. In-gap Electronic Structure of $\mathrm{LaAlO}_{3}-\mathrm{SrTiO}_{3}$ Heterointerfaces Investigated by Soft X-ray Spectroscopy. Phys. Rev. B 2011, 84, 245121.

(250) Santander-Syro, A. F. et al. Two-dimensional Electron Gas with Universal Subbands at the Surface of $\mathrm{SrTiO}_{3}$. Nature 2011, 469, 189-193.

(251) Meevasana, W.; King, P. D. C.; He, R. H.; Mo, S.-K.; Hashimoto, M.; Tamai, A.; Songsiriritthigul, P.; Baumberger, F.; Shen, Z.-X. Creation and control of a twodimensional electron liquid at the bare SrTiO surface. Nat. Mater. 2011, 10, 114-118.

(252) McKeown Walker, A., S. ae; Bruno, F.; Tamai, A.; Kim, T.; Hoesch, M.; Shi, M.; Bahramy, M.; King, P.; Baumberger, F. Control of a Two-Dimensional Electron Gas on $\mathrm{SrTiO}_{3}(111)$ by Atomic Oxygen. Phys. Rev. Lett. 2014, 113, 177601.

(253) Dudy, L.; Sing, M.; Scheiderer, P.; Denlinger, J. D.; Schütz, P.; Gabel, J.; Buchwald, M.; Schlueter, C.; Lee, T.-L.; Claessen, R. In Situ Control of Separate Electronic Phases on $\mathrm{SrTiO}_{3}$ Surfaces by Oxygen Dosing. Adv. Mater. 2016, 28, 7443-7449. 
(254) Wang, Z. et al. Tailoring the Nature and Strength of Electron-Phonon Interactions in the $\mathrm{SrTiO}_{3}(001)$ 2D Electron Liquid. Nat. Mater. 2016, 15, 835-839.

(255) Chen, C.; Avila, J.; Frantzeskakis, E.; Levy, A.; Asensio, M. C. Observation of a twodimensional liquid of Frohlich polarons at the bare $\mathrm{SrTiO}_{3}$ surface. Nat. Commun. $\mathbf{2 0 1 5}, 6,8585$.

(256) Cancellieri, C.; Mishchenko, A. S.; Aschauer, U.; Filippetti, A.; Faber, C.; Barišić, O. S.; Rogalev, V. A.; Schmitt, T.; Nagaosa, N.; Strocov, V. N. Polaronic Metal state at the $\mathrm{LaAlO}_{3} / \mathrm{SrTiO}_{3}$ interface. Nat. Commun. 2016, 7, 10386.

(257) Nie, Y. F.; Di Sante, D.; Chatterjee, S.; King, P. D. C.; Uchida, M.; Ciuchi, S.; Schlom, D. G.; Shen, K. M. Formation and Observation of a Quasi-Two-Dimensional $d_{x y}$ Electron Liquid in Epitaxially Stabilized $\mathrm{Sr}_{2-x} \mathrm{La}_{x} \mathrm{TiO}_{4}$ Thin Films. Phys. Rev. Lett. 2015, 115, 096405.

(258) Moser, S.; Moreschini, L.; Jaćimović, J.; Barišić, O. S.; Berger, H.; Magrez, A.; Chang, Y. J.; Kim, K. S.; Bostwick, A.; Rotenberg, E.; Forró, L.; Grioni, M. Tunable Polaronic Conduction in Anatase $\mathrm{TiO}_{2}$. Phys. Rev. Lett. 2013, 110, 196403.

(259) Riley, J. M.; Caruso, F.; Verdi, C.; Duffy, L. B.; Watson, M. D.; Bawden, L.; Volckaert, K.; Laan, G. v. d.; Hesjedal, T.; Hoesch, M.; Giustino, F.; King, P. D. C. Crossover from Lattice to Plasmonic Polarons of a Spin-Polarised Electron Gas in Ferromagnetic EuO. Nat. Commun. 2018, 9, 2305.

(260) Verdi, C.; Caruso, F.; Giustino, F. Origin of the Crossover from Polarons to Fermi Liquids in Transition Metal Oxides. Nat. Commun. 2017, 8, 15769.

(261) Thompson, T. L.; Yates, J. T. Surface Science Studies of the Photoactivation of $\mathrm{TiO}_{2}$ New Photochemical Processes. Chem. Rev. 2006, 106, 4428-4453. 
(262) Zhang, S. X.; Kundaliya, D. C.; Yu, W.; Dhar, S.; Young, S. Y.; SalamancaRiba, L. G.; Ogale, S. B.; Vispute, R. D.; Venkatesan, T. Niobium Doped $\mathrm{TiO}_{2}$ : Intrinsic Transparent Metallic Anatase versus Highly Resistive Rutile Phase. J. Appl. Phys. 2007, 102, 013701.

(263) Chen, C.; Avila, J.; Frantzeskakis, E.; Levy, A.; Asensio, M. C. Observation of a TwoDimensional Liquid of Fröhlich Polarons at the Bare $\mathrm{SrTiO}_{3}$ Surface. Nat. Commun. 2015, 6, 8-15.

(264) Le Fèvre, P.; Danger, J.; Magnan, H.; Chandesris, D.; Jupille, J.; Bourgeois, S.; Arrio, M. A.; Gotter, R.; Verdini, A.; Morgante, A. Stoichiometry-related Auger Lineshapes in Titanium Oxides: Influence of Valence-Band Profile and of Coster-Krönig Processes. Phys. Rev. B 2004, 69, 155421.

(265) Selcuk, S.; Selloni, A. Facet-dependent Trapping and Dynamics of Excess Electrons at Anatase $\mathrm{TiO}_{2}$ Surfaces and Aqueous Interfaces. Nat. Mater. 2016, 15, 1107-1112.

(266) Hashimoto, M.; Vishik, I. M.; He, R.-H.; Devereaux, T. P.; Shen, Z.-X. Energy Gaps in High-Transition-Temperature Cuprate Superconductors. Nat. Phys 2014, 10, 483495.

(267) Kordyuk, A. A. Pseudogap from ARPES experiment: Three gaps in Cuprates and Topological Superconductivity. Low Temp. Phys. 2015, 41, 319-341.

(268) Vishik, I. M. Photoemission Perspective on pseudogap, Superconducting Fluctuations, and Charge Order in Cuprates: a Review of Recent Progress. Rep. Prog. Phys. 2018, $81,062501$.

(269) Bergemann, C.; Mackenzie, A. P.; Julian, S. R.; Forsythe, D.; Ohmichi, E. Quasi-twodimensional Fermi Liquid Properties of the Unconventional Superconductor $\mathrm{Sr}_{2} \mathrm{RuO}_{4}$. Adv. Phys. 2003, 52, 639-725. 
(270) Mackenzie, A. P.; Julian, S. R.; Diver, A. J.; McMullan, G. J.; Ray, M. P.; Lonzarich, G. G.; Maeno, Y.; Nishizaki, S.; Fujita, T. Quantum Oscillations in the Layered Perovskite Superconductor $\mathrm{Sr}_{2} R u \mathrm{O}_{4}$. Phys. Rev. Lett. 1996, 76, 3786-3789.

(271) Mackenzie, A. P.; Maeno, Y. The Superconductivity of $S r_{2} R u O_{4}$ and the Physics of Spin-Triplet Pairing. Rev. Mod. Phys. 2003, 75, 657-712.

(272) Mackenzie, A. P.; Scaffidi, T.; Hicks, C. W.; Maeno, Y. Even odder after twentythree years: the Superconducting Order Parameter Puzzle of $S r_{2} R u O_{4}$. npj Quantum Mater. 2017, 2, 40.

(273) Damascelli, A.; Lu, D. H.; Shen, K. M.; Armitage, N. P.; Ronning, F.; Feng, D. L.; Kim, C.; Shen, Z.-X.; Kimura, T.; Tokura, Y.; Mao, Z. Q.; Maeno, Y. Fermi Surface,Surface States,and Surface Reconstruction in $\mathrm{Sr}_{2} \mathrm{RuO} \mathrm{O}_{4}$. Phys. Rev. Lett. 2000, $85,5194-5197$.

(274) Tamai, A. et al. High-Resolution Photoemission on $\mathrm{Sr}_{2} \mathrm{RuO}_{4}$ Reveals CorrelationEnhanced Effective Spin-Orbit Coupling and Dominantly Local Self-Energies. Phys. Rev. X 2019, 9, 021048.

(275) Singh, D. J. Relationship of $\mathrm{Sr}_{2} \mathrm{RuO}_{4}$ to the Superconducting Layered Cuprates. Phys. Rev. B 1995, 52, 1358-1361.

(276) Zhang, G.; Gorelov, E.; Sarvestani, E.; Pavarini, E. Fermi Surface of $\mathrm{Sr}_{2} \mathrm{RuO}_{4}$ : Spin-Orbit and Anisotropic Coulomb Interaction Effects. Phys. Rev. Lett. 2016, 116, 106402.

(277) Ingle, N. J. C.; Shen, K. M.; Baumberger, F.; Meevasana, W.; Lu, D. H.; Shen, Z.X.; Damascelli, A.; Nakatsuji, S.; Mao, Z. Q.; Maeno, Y.; Kimura, T.; Tokura, Y. Quantitative Analysis of $\mathrm{Sr}_{2} \mathrm{RuO}_{4}$ Angle-Resolved Photoemission Spectra: Manybody Interactions in a model Fermi liquid. Phys. Rev. B 2005, 72, 205114. 
(278) Iwasawa, H.; Aiura, Y.; Saitoh, T.; Yoshida, Y.; Hase, I.; Ikeda, S. I.; Bando, H. Fermi Surfaces and Kink in the Energy Dispersion of $\mathrm{Sr}_{2} \mathrm{RuO}_{4}$. Proceedings of the 18th International Symposium on Superconductivity (ISS 2005), 2006, 445-448, 7376.

(279) Iwasawa, H.; Yoshida, Y.; Hase, I.; Koikegami, S.; Hayashi, H.; Jiang, J.; Shimada, K.; Namatame, H.; Taniguchi, M.; Aiura, Y. Interplay among Coulomb Interaction, SpinOrbit Interaction, and Multiple Electron-Boson Interactions in $\mathrm{Sr}_{2} \mathrm{RuO} \mathrm{O}_{4}$. Phys. Rev. Lett. 2010, 105, 226406.

(280) Iwasawa, H.; Yoshida, Y.; Hase, I.; Shimada, K.; Namatame, H.; Taniguchi, M.; Aiura, Y. High-Energy Anomaly in the Band Dispersion of the Ruthenate Superconductor. Phys. Rev. Lett. 2012, 109, 066404.

(281) Zabolotnyy, V. B.; Evtushinsky, D. V.; Kordyuk, A. P.; Kim, T. K.; Carleschi, E.; Doyle, B. P.; Fittipaldi, R.; Cuoco, M.; Vecchione, A.; Borisenko, S. V. Renormalized Band Structure of $\mathrm{Sr}_{2} \mathrm{RuO}_{4}$ A Quasiparticle Tight-Binding Approach. J. Electron Spectros. Relat. Phenomena 2013, 191, 48-53.

(282) Burganov, B.; Adamo, C.; Mulder, A.; Uchida, M.; King, P. D. C.; Harter, J. W.; Shai, D. E.; Gibbs, A. S.; Mackenzie, A. P.; Uecker, R.; Bruetzam, M.; Beasley, M. R.; Fennie, C. J.; Schlom, D. G.; Shen, K. M. Strain Control of Fermiology and Many-Body Interactions in Two-Dimensional Ruthenates. Phys. Rev. Lett. 2016, 116, 197003.

(283) Steppke, A.; Zhao, L. S.; Barber, M. E.; Scaffidi, T.; Jerzembeck, F.; Rosner, H.; Gibbs, A. S.; Maeno, Y.; Simon, S. H.; Mackenzie, A. P.; Hicks, C. W. Strong peak in Tc of $\mathrm{Sr}_{2} \mathrm{RuO}_{4}$ under Uniaxial Pressure. Science 2017, 355, 148, eaaf9398.

(284) Shen, K. M.; Kikugawa, N.; Bergemann, C.; Balicas, L.; Baumberger, F.; Meevasana, W.; Ingle, N. J. C.; Maeno, Y.; Shen, Z.-X.; Mackenzie, A. P. Evolution of 
the Fermi Surface and Quasiparticle Renormalization through a van Hove Singularity in $\mathrm{Sr}_{2-y} \mathrm{La}_{y} R u \mathrm{O}_{4}$. Phys. Rev. Lett. 2007, 99, 187001.

(285) Sunko, V.; Abarca Morales, E.; Marković, I.; Barber, M. E.; Milosavljević, D.; Mazzola, F.; Sokolov, D. A.; Kikugawa, N.; Cacho, C.; Dudin, P.; Rosner, H.; Hicks, C. W.; King, P. D. C.; Mackenzie, A. P. Direct Observation of a Uniaxial Stress-driven Lifshitz Transition in $\mathrm{Sr}_{2} \mathrm{RuO}_{4}$. npj Quantum Mater. 2019, 4, 46.

(286) Monkman, E. J.; Adamo, C.; Mundy, J. A.; Shai, D. E.; Harter, J. W.; Shen, D.; Burganov, B.; Muller, D. A.; Schlom, D. G.; Shen, K. M. Quantum Many-Body Interactions in Digital Oxide Superlattices. Nat. Mater. 2012, 11, 855-859.

(287) King, P. D. C.; Wei, H. I.; Nie, Y. F.; Uchida, M.; Adamo, C.; Zhu, S.; He, X.; Božović, I.; Schlom, D. G.; Shen, K. M. Atomic-scale Control of Competing Electronic Phases in Ultrathin $\mathrm{LaNiO}_{3}$. Nat. Nanotechnol. 2014, 9, 443-447.

(288) Lee, J. J.; Schmitt, F. T.; Moore, R. G.; Johnston, S.; Cui, Y.-T.; Li, W.; Yi, M.; Liu, Z. K.; Hashimoto, M.; Zhang, Y.; Lu, D. H.; Devereaux, T. P.; Lee, D.-H.; Shen, Z.-X. Interfacial Mode Coupling as the Origin of the Enhancement of $T_{c}$ in FeSe films on $\mathrm{SrTiO}_{3}$. Nature 2014, 515, 245-248.

(289) Yi, M.; Lu, D.; Chu, J.-H.; Analytis, J. G.; Sorini, A. P.; Kemper, A. F.; Moritz, B.; Mo, S.-K.; Moore, R. G.; Hashimoto, M.; Lee, W.-S.; Hussain, Z.; Devereaux, T. P.; Fisher, I. R.; Shen, Z.-X. Symmetry-breaking Orbital Anisotropy observed for Detwinned $\mathrm{Ba}\left(\mathrm{Fe}_{1-x} \mathrm{Co}_{x}\right)_{2} \mathrm{As}_{2}$ above the spin density wave transition. Proc. Natl. Acad. Sci. U.S.A. 2011, 108, 6878-6883.

(290) Kim, Y.; Oh, H.; Kim, C.; Song, D.; Jung, W.; Kim, B.; Choi, H. J.; Kim, C.; Lee, B.; Khim, S.; Kim, H.; Kim, K.; Hong, J.; Kwon, Y. Electronic Structure of detwinned $\mathrm{BaFe}_{2} \mathrm{As}_{2}$ from Photoemission and First Principles. Phys. Rev. B 2011, 83, 064509. 
(291) Watson, M. D.; Haghighirad, A. A.; Rhodes, L. C.; Hoesch, M.; Kim, T. K. Electronic Anisotropies revealed by detwinned Angle-Resolved Photoemission Spectroscopy Measurements of FeSe. New J. Phys 2017, 19, 103021.

(292) Pfau, H.; Rotundu, C. R.; Palmstrom, J. C.; Chen, S. D.; Hashimoto, M.; Lu, D.; Kemper, A. F.; Fisher, I. R.; Shen, Z.-X. Detailed Band Structure of twinned and detwinned $\mathrm{BaFe}_{2} \mathrm{As}_{2}$ studied with Angle-Resolved Photoemission Spectroscopy. Phys. Rev. B 2019, 99 .

(293) Flötotto, D.; Bai, Y.; Chan, Y.-H.; Chen, P.; Wang, X.; Rossi, P.; Xu, C.-Z.; Zhang, C.; Hlevyack, J. A.; Denlinger, J. D.; Hong, H.; Chou, M.-Y.; Mittemeijer, E. J.; Eckstein, J. N.; Chiang, T.-C. In Situ Strain Tuning of the Dirac Surface States in $\mathrm{Bi}_{2} \mathrm{Se}_{3}$ Films. Nano Lett. 2018, 18, 5628-5632.

(294) Riccò, S.; Kim, M.; Tamai, A.; McKeown Walker, S.; Bruno, F. Y.; Cucchi, I.; Cappelli, E.; Besnard, C.; Kim, T. K.; Dudin, P.; Hoesch, M.; Gutmann, M. J.; Georges, A.; Perry, R. S.; Baumberger, F. In Situ Strain Tuning of the Metal-InsulatorTransition of $\mathrm{Ca}_{2} \mathrm{RuO}_{4}$ in Angle-Resolved Photoemission Experiments. Nat. Commun. 2018, 9, 4535 .

(295) Lupi, S. et al. A Microscopic View on the Mott Transition in Chromium-doped $\mathrm{V}_{2} \mathrm{O}_{3}$. Nat. Commun. 2010, 1, 105.

(296) Eriksson, M.; Van Der Veen, J. F.; Quitmann, C. Diffraction-limited Storage Rings. A Window to the Science of Tomorrow. J. Synchrotron Radiat. 2014, 21, 837-842.

(297) Rotenberg, E.; Bostwick, A. Microarpes and Nanoarpes at Diffraction-limited Light Sources: Opportunities and Performance Gains. J. Synchrotron Radiat. 2014, 21, $1048-1056$.

(298) Kirkpatrick, P.; Baez, A. V. Formation of Optical Images by X-Rays. J. Opt. Soc. Am. 1948, 38, 766-774. 
(299) Dudin, P.; Lacovig, P.; Fava, C.; Nicolini, E.; Bianco, A.; Cautero, G.; Barinov, A. Angle-resolved Photoemission Spectroscopy and Imaging with a Submicrometre Probe at the SPECTROMICROSCOPY-3.2L Beamline of Elettra. J. Synchrotron Radiat. 2010, $17,445-450$.

(300) Koch, R. J.; Jozwiak, C.; Bostwick, A.; Stripe, B.; Cordier, M.; Hussain, Z.; Yun, W.; Rotenberg, E. Nano focusing of Soft X-rays by a New Capillary Mirror Optic. Synchr. Rad. News 2018, 31, 50-52.

(301) Cucini, R. et al. Coherent Narrowband Light Source for Ultrafast Photoelectron Spectroscopy in the 17-31 eV Photon Energy Range. Struct. Dyn. 2020, 7, 014303.

(302) He, Y.; Vishik, I. M.; Yi, M.; Yang, S.; Liu, Z.; Lee, J. J.; Chen, S.; Rebec, S. N.; Leuenberger, D.; Zong, A.; Jefferson, C. M.; Moore, R. G.; Kirchmann, P. S.; Merriam, A. J.; Shen, Z. X. High resolution Angle Resolved Photoemission with Tabletop 11 eV Laser. Rev. Sci. Instrum. 2016, 87, 011301.

(303) Zhou, X.; He, S.; Liu, G.; Zhao, L.; Yu, L.; Zhang, W. New Developments in Laserbased Photoemission Spectroscopy and its Scientific Applications: a Key Issues Review. Rep. Prog. Phys. 2018, 81, 062101.

(304) Frietsch, B.; Carley, R.; Döbrich, K.; Gahl, C.; Teichmann, M.; Schwarzkopf, O.; Wernet, P.; Weinelt, M. A High-order Harmonic Generation Apparatus for Time- and Angle-Resolved Photoelectron Spectroscopy. Rev. Sci. Instrum. 2013, 84, 075106.

(305) Thieme, J., Schmahl, D., Rudolph, D., Umbach, E., Eds. X-Ray Microscopy and Spectromicroscopy; Springer, Berlin, Heidelberg: Würzburg, 1998.

(306) Kutnyakhov, D. et al. Time- and Momentum-resolved Photoemission Studies using Time-Of-Flight Momentum Microscopy at a Free-Electron Laser. Rev. Sci. Instrum. 2020, 91, 013109 . 
(307) Maklar, J.; Dong, S.; Beaulieu, S.; Pincelli, T.; Dendzik, M.; Windsor, Y. W.; Xian, R. P.; Wolf, M.; Ernstorfer, R.; Rettig, L. A quantitative comparison of time-offlight momentum microscopes and hemispherical analyzers for time-resolved ARPES experiments. arXiv:2008.05829 [physics] 2020, arXiv: 2008.05829.

(308) Schönhense, G.; Medjanik, K.; Elmers, H. J. Space-, Time- and Spin-resolved Photoemission. J. Electron Spectros. Relat. Phenomena 2015, 200, 94-118.

(309) Schönhense, G.; Medjanik, K.; Babenkov, S.; Vasilyev, D.; Ellguth, M.; Fedchenko, O.; Chernov, S.; Schönhense, B.; Elmers, H. J. Momentum-transfer model of valence-band photoelectron diffraction. Comm. Phys. 2020, 3, 45; 1-13.

(310) Locatelli, A.; Menteş, T. O.; Niño, M. Á.; Bauer, E. Image Blur and Energy Broadening Effects in XPEEM. Ultramicroscopy 2011, 111, 1447-1454.

(311) Merkel, M.; Escher, M.; Settemeyer, J.; Funnemann, D.; Oelsner, A.; Ziethen, C.; Schmidt, O.; Klais, M.; Schönhense, G. Microspectroscopy and Spectromicroscopy with Photoemission Electron Microscopy using a New Kind of Imaging Energy Filter. Surf. Sci. 2001, 480, 196-202.

(312) Salmeron, M.; Schlögl, R. Ambient Pressure Photoelectron Spectroscopy : A New Tool for Surface Science and Nanotechnology. Surf. Sci. Rep. 2008, 63, 169-199.

(313) Salmeron, M. From Surfaces to Interfaces: Ambient Pressure XPS and Beyond. Topics in Catalysis 2018, 61, 2044-2051.

(314) Wang, C. H.; Chang, S. T.; Chen, S. Y.; Yang, Y. W. New Ambient Pressure X-ray Photoelectron Spectroscopy Endstation at Taiwan Light Source. AIP Conf. Proceedings 2019, 2054, 1-7.

(315) Velasco-Velez, J. J.; Pfeifer, V.; Hävecker, M.; Weatherup, R. S.; Arrigo, R.; Chuang, C. H.; Stotz, E.; Weinberg, G.; Salmeron, M.; Schlögl, R.; Knop-Gericke, A. 
Photoelectron Spectroscopy at the Graphene-Liquid Interface Reveals the Electronic Structure of an Electrodeposited Cobalt/Graphene Electrocatalyst. Angew. Chem. 2015, 54, 14554-14558.

(316) Newberg, J. T.; Åhlund, J.; Arble, C.; Goodwin, C.; Khalifa, Y.; Broderick, A. A Lab-based Ambient Pressure X-ray Photoelectron Spectrometer with Exchangeable Analysis Chambers. Rev. Sci. Instrum 2015, 86, 085113.

(317) Hellmann, S.; Rossnagel, K.; Marczynski-Bühlow, M.; Kipp, L. Vacuum Space-Charge Effects in Solid-State Photoemission. Phys. Rev. B 2009, 79, 035402.

(318) Hellmann, S.; Sohrt, C.; Beye, M.; Rohwer, T.; Sorgenfrei, F.; Marczynski-Bühlow, M.; Kalläne, M.; Redlin, H.; Hennies, F.; Bauer, M.; Föhlisch, A.; Kipp, L.; Wurth, W.; Rossnagel, K. Time-resolved X-ray Photoelectron Spectroscopy at FLASH. New J. Phys. 2012, 14, 013062.

(319) Wang, H.; Xu, Y.; Ulonska, S.; Robinson, J. S.; Ranitovic, P.; Kaindl, R. A. Bright high-repetition-rate source of narrowband extreme-ultraviolet harmonics beyond 22 eV. Nat Commun 2015, 6, 7459 .

(320) Crepaldi, A.; Roth, S.; Gatti, G.; Arrell, C.; Ojeda, J.; van Mourik, F.; Bugnon, P.; Magrez, A.; Berger, H.; Chergui, M.; Grioni, M. Time-resolved ARPES at LACUS: Band Structure and Ultrafast Electron Dynamics of Solids. CHIMIA Int. Journ. Chem. 2017, $71,273-277$.

(321) Corder, C.; Zhao, P.; Bakalis, J.; Li, X.; Kershis, M. D.; Muraca, A. R.; White, M. G.; Allison, T. K. Ultrafast extreme ultraviolet photoemission without space charge. Struct. Dyn. 2018, 5, 054301.

(322) Puppin, M.; Deng, Y.; Nicholson, C. W.; Feldl, J.; Schröter, N. B. M.; Vita, H.; Kirchmann, P. S.; Monney, C.; Rettig, L.; Wolf, M.; Ernstorfer, R. Time- and angle- 
resolved photoemission spectroscopy of solids in the extreme ultraviolet at $500 \mathrm{kHz}$ repetition rate. Rev. Sci. Instrum. 2019, 90, 023104.

(323) Buss, J. H.; Wang, H.; Xu, Y.; Maklar, J.; Joucken, F.; Zeng, L.; Stoll, S.; Jozwiak, C.; Pepper, J.; Chuang, Y.-D.; Denlinger, J. D.; Hussain, Z.; Lanzara, A.; Kaindl, R. A. A Setup for Extreme-Ultraviolet Ultrafast Angle-Resolved Photoelectron Spectroscopy at 50-kHz Repetition Rate. Rev. Sci. Instrum. 2019, 90, 023105.

(324) Sie, E. J.; Rohwer, T.; Lee, C.; Gedik, N. Time-resolved XUV ARPES with tunable 24-33 eV laser pulses at $30 \mathrm{meV}$ resolution. Nat. Commun. 2019, 10, 3535.

(325) He, Y.; Hashimoto, M.; Song, D.; Chen, S.-D.; He, J.; Vishik, I. M.; Moritz, B.; Lee, D.-H.; Nagaosa, N.; Zaanen, J.; Devereaux, T. P.; Yoshida, Y.; Eisaki, H.; Lu, D. H.; Shen, Z.-X. Rapid Change of Superconductivity and Electron-Phonon Coupling through Critical Doping in Bi-2212. Science 2018, 362, 62-65.

(326) Na, M. X.; Mills, A. K.; Boschini, F.; Michiardi, M.; Nosarzewski, B.; Day, R. P.; Razzoli, E.; Sheyerman, A.; Schneider, M.; Levy, G.; Zhdanovich, S.; Devereaux, T. P.; Kemper, A. F.; Jones, D. J.; Damascelli, A. Direct Determination of Mode-projected Electron-Phonon Coupling in the Time Domain. Science 2019, 366, 1231-1236.

(327) Smallwood, C. L.; Hinton, J. P.; Jozwiak, C.; Zhang, W.; Koralek, J. D.; Eisaki, H.; Lee, D.-H.; Orenstein, J.; Lanzara, A. Tracking Cooper Pairs in a Cuprate Superconductor by Ultrafast Angle-Resolved Photoemission. Science 2012, 336, 1137-1139.

(328) Nicholson, C. W.; Lücke, A.; Schmidt, W. G.; Puppin, M.; Rettig, L.; Ernstorfer, R.; Wolf, M. Beyond the Molecular Movie: Dynamics of Bands and Bonds during a Photoinduced Phase Transition. Science 2018, 362, 821-825.

(329) Rohwer, T.; Hellmann, S.; Wiesenmayer, M.; Sohrt, C.; Stange, A.; Slomski, B.; Carr, A.; Liu, Y.; Avila, L. M.; Kalläne, M.; Mathias, S.; Kipp, L.; Rossnagel, K.; 
Bauer, M. Collapse of Long-Range Charge Order Tracked by Time-Resolved Photoemission at High Momenta. Nature 2011, 471, 490-493.

(330) Hellmann, S.; Rohwer, T.; Kalläne, M.; Hanff, K.; Sohrt, C.; Stange, A.; Carr, A.; Murnane, M. M.; Kapteyn, H. C.; Kipp, L.; Bauer, M.; Rossnagel, K. Time-domain Classification of Charge-Density-Wave Insulators. Nat. Commun. 2012, 3, 1069.

(331) Wang, Y. H.; Steinberg, H.; Jarillo-Herrero, P.; Gedik, N. Observation of FloquetBloch States on the Surface of a Topological Insulator. Science 2013, 342, 453-457.

(332) Smallwood, C. L.; Kaindl, R. A.; Lanzara, A. Ultrafast Angle-Resolved Photoemission Spectroscopy of Quantum Materials. EPL 2016, 115, 27001.

(333) Schlom, D. G.; Chen, L.-Q.; Pan, X.; Schmehl, A.; Zurbuchen, M. A. A Thin Film Approach to Engineering Functionality into Oxides. J. Am. Ceram. Soc. 2008, 91, $2429-2454$.

(334) Belopolski, I. et al. A Novel Artificial Condensed Matter Lattice and a New Platform for One-Dimensional Topological Phases. Sci. Adv. 2017, 3, 12-15.

(335) Smyth, D. M. The Defect Chemistry of Metal Oxides; Oxford University Press: Oxford, 2000.

(336) Lombardi, F.; Lodi, A.; Ma, J.; Liu, J.; Slota, M.; Narita, A.; Myers, W. K.; Müllen, K.; Feng, X.; Bogani, L. Quantum Units from the Topological Engineering of Molecular Graphenoids. Science 2019, 366, 1107-1110.

(337) Mo, S.-K. Angle-Resolved Photoemission Spectroscopy for the Study of TwoDimensional Materials. Nano Converg. 2017, 4, 6.

(338) Orgiani, P.; Bigi, C.; Kumar Das, P.; Fujii, J.; Ciancio, R.; Gobaut, B.; Galdi, A.; Sacco, C.; Maritato, L.; Torelli, P.; Panaccione, G.; Vobornik, I.; Rossi, G. Struc- 
tural and Electronic Properties of $\mathrm{Bi}_{2} 2 \mathrm{Se}_{3}$ Topological Insulator Thin Films grown by Pulsed Laser Deposition. Appl. Phys. Lett. 2017, 110, 171601.

(339) Koch, R.; Moser, S.; Ulstrup, S.; Moreschini, L.; Jozwiak, C.; Bostwick, A.; Rotenberg, E. The new NanoARPES at MAESTRO of the ALS. APS March Meeting Abstracts. 2017; p L36.007.

(340) Lee, M.-I.; Barragán, A.; Nair, M. N.; Jacques, V. L. R.; Le Bolloc'h, D.; Fertey, P.; Jemli, K.; Lédée, F.; Trippé-Allard, G.; Deleporte, E.; Taleb-Ibrahimi, A.; Tejeda, A. First Determination of the Valence Band Dispersion of $\mathrm{CH}_{3} \mathrm{NH}_{3} \mathrm{PbI}_{3}$ hybrid organic-inorganic perovskite. J. Phys. D 2017, 50, $26 \mathrm{LT} 02$. 
Graphical TOC Entry

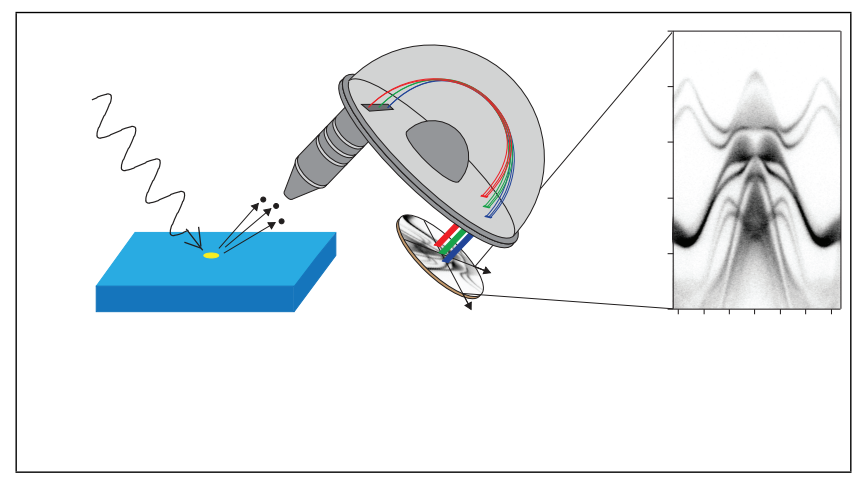

